Florida International University

FIU Digital Commons

$10-31-2018$

\title{
Social Dominance Orientation and Emotion Regulation: A Parallel Multiple Mediator Model of Instigated Incivility Moderated By Workgroup Civility Climate
}

\author{
Fabiana Brunetta \\ drfbrunetta@gmail.com
}

Follow this and additional works at: https://digitalcommons.fiu.edu/etd

Part of the Industrial and Organizational Psychology Commons, Organizational Behavior and Theory Commons, Personality and Social Contexts Commons, Social Psychology Commons, Social Psychology and Interaction Commons, and the Training and Development Commons

\section{Recommended Citation}

Brunetta, Fabiana, "Social Dominance Orientation and Emotion Regulation: A Parallel Multiple Mediator Model of Instigated Incivility Moderated By Workgroup Civility Climate" (2018). FIU Electronic Theses and Dissertations. 3907.

https://digitalcommons.fiu.edu/etd/3907

This work is brought to you for free and open access by the University Graduate School at FIU Digital Commons. It has been accepted for inclusion in FIU Electronic Theses and Dissertations by an authorized administrator of FIU Digital Commons. For more information, please contact dcc@fiu.edu. 


\title{
FLORIDA INTERNATIONAL UNIVERSITY
}

Miami, Florida

\section{SOCIAL DOMINANCE ORIENTATION AND EMOTION REGULATION: A PARALLEL MULTIPLE MEDIATOR MODEL OF INSTIGATED INCIVILITY MODERATED BY WORKGROUP CIVILITY CLIMATE}

\author{
A dissertation submitted in partial fulfillment of \\ the requirements for the degree of \\ DOCTOR OF EDUCATION \\ in \\ ADULT EDUCATION AND HUMAN RESOURCE DEVELOPMENT \\ by \\ Fabiana Brunetta
}


To: Dean Michael R. Heithaus

College of Arts, Sciences and Education

This dissertation, written by Fabiana Brunetta, and entitled Social Dominance Orientation and Emotion Regulation: A Parallel Multiple Mediator Model of Instigated Incivility Moderated by Workgroup Civility Climate, having been approved in respect to style and intellectual content, is referred to you for judgment.

We have read this dissertation and recommend that it be approved.

Tonette S. Rocco

$\begin{array}{r}\hline \text { Joanne Sanders-Reio } \\ \hline \text { Andrea J. Queeley }\end{array}$

Thomas G. Reio, Jr., Major Professor

Date of Defense: October 31, 2018

The dissertation of Fabiana Brunetta is approved.

Dean Michael R. Heithaus

College of Arts, Sciences and Education

Andrés G. Gil

Vice President for Research and Economic Development and Dean of the University Graduate School

Florida International University, 2018 
(C) Copyright 2018 by Fabiana Brunetta

All rights reserved. 


\section{DEDICATION}

I dedicate this dissertation to my parents Giuliana and Paolo, my children Elizabeth and Catherine, and my husband Guillermo. 


\section{ACKNOWLEDGMENTS}

My unreserved appreciation goes to Dr. Thomas Reio, Jr. whose intellectual and emotional support made this dissertation possible. Your friendship and mentorship went above and beyond the call of academic duty. A very special thank you to Dr. Joanne Sanders-Reio, who believed in my ability and encouraged me to pursue this academic endeavor. I am also indebted to Dr. Tonette Rocco for providing a generous amount of support with careful edits. Thank you to Dr. Andrea Queeley for her thoughtful feedback and theoretical insights. A sincere thank you to Dr. Leonard Bliss and Dr. Linda Bliss, who have supported me in many ways throughout this journey.

To my husband Guillermo, I have proof that your love moves mountains. Giuliana, you showed me the value of grit and I am proud and honored to call you my mother. To my daughter Elizabeth, your strength and sense of humor bring hope to the most challenging of days. To my daughter Catherine, your kindness and work ethic will never cease to inspire me. To my brother Federico and my sister Lina, thank you for always being just a phone call away. To my niece Nicole, I love being your "crazy Zia Faby." To my niece Stella, thank you for motivating me to finish my dissertation this year. To my new family members, Gino, Jackie, and Nancy, what would I have done without you?

A special thank you to my friends Alessandra and Alexandra. Your unconditional support and words of wisdom are priceless. Thank you to Chaundra, Carolyn, Keisha, and Craig - graduate school would not have been the same without you. 


\section{ABSTRACT OF THE DISSERTATION \\ SOCIAL DOMINANCE ORIENTATION AND EMOTION REGULATION: A \\ PARALLEL MULTIPLE MEDIATOR MODEL OF INSTIGATED INCIVILITY MODERATED BY WORKGROUP CIVILITY CLIMATE}

by

Fabiana Brunetta

Florida International University, 2018

Miami, Florida

Professor Thomas G. Reio, Jr., Major Professor

While most of the existing research on the topic of workplace incivility has focused upon its consequences on employee and organizational well-being, researchers are recognizing the need for research on predictors, mediators, and moderators of uncivil workplace behavior. The current study contributes to this new wave of workplace incivility research by emphasizing the links among variables not previously explored in incivility research. This nonexperimental correlational study $(N=1027)$ developed and tested a parallel multiple mediator model of instigated incivility. The model examined the mediation of the emotion regulation strategies - cognitive reappraisal and expressive suppression - on the relation of two types of social dominance orientation - intergroup dominance (SDO-D) and intergroup antiegalitarianism (SDO-E) - on the outcome of instigated incivility, and tested the moderating effects of workgroup civility climate on the paths of the proposed model.

A self-report survey battery was administered using the Internet to a sample drawn from Amazon's Mechanical Turk worker population. Hypotheses were tested 
through structural equation modeling analytic procedures. Findings suggest that intergroup dominance (SDO-D) increases instigated incivility and the relationship is not moderated by workgroup civility climate norms. In contrast, intergroup antiegalitarianism (SDO-E) decreases instigated incivility. Further, this study found that SDO-D had an indirect effect on instigated incivility through the emotion regulation strategy of expressive suppression. Additional findings suggest that the emotion regulation strategy of cognitive reappraisal has the potential to reduce uncivil workplace behavior. Future research was proposed to test the model examined in this study in different cultural settings, with additional mediators and moderators, and longitudinally. The practical findings suggest that HRD practitioners may find emotion regulation and civility trainings useful to reduce the likelihood of uncivil workplace behavior. 


\section{TABLE OF CONTENTS}

CHAPTER

PAGE

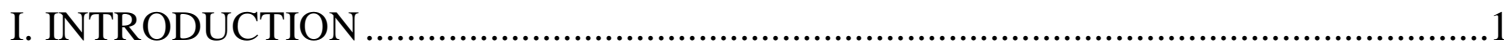

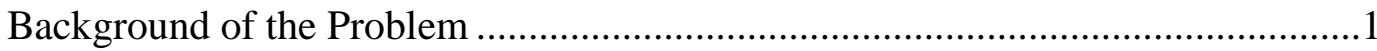

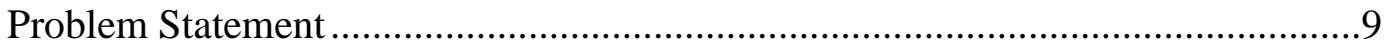

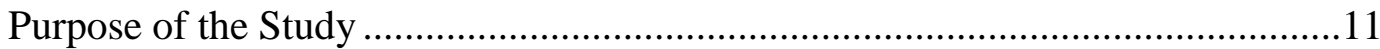

Research Questions and Hypotheses ............................................................12

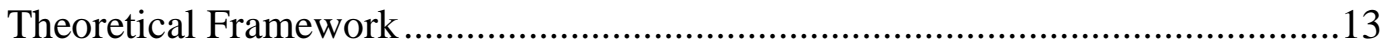

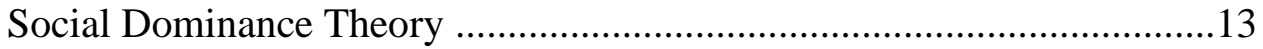

Emotion Regulation Framework ......................................................... 16

Hypothesized Model of Instigated Incivility .................................................19

Significance of the Study ......................................................................23

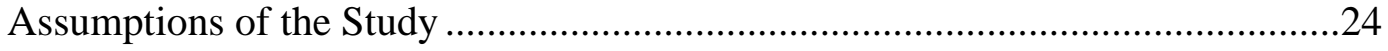

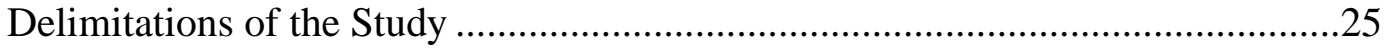

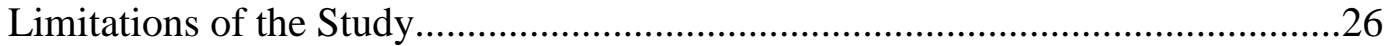

Definition of Terms ..................................................................................28

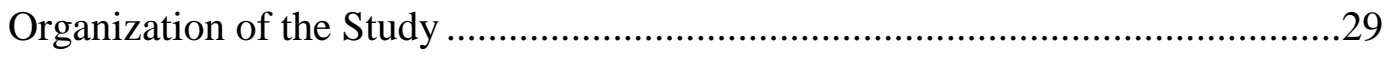

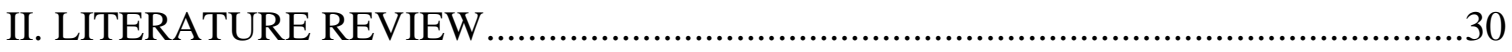

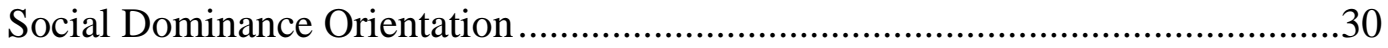

Social Dominance Orientation as a - Unidimensional Construct..............31

Organizational Studies .........................................................33

Workplace Behavior ..........................................................35

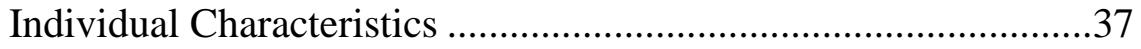

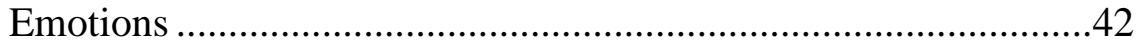

Intergroup Dominance and Intergroup Antiegalitarianism .....................46

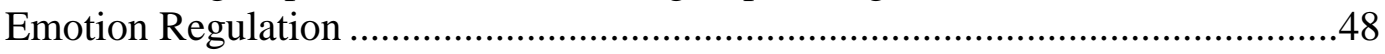

Organizational Display Rules .....................................................51

Emotion Regulation Strategies ......................................................52

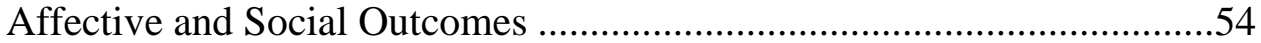

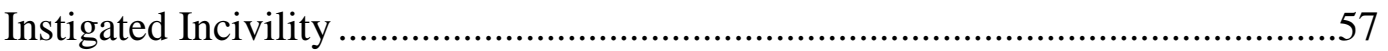

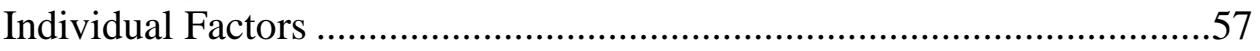

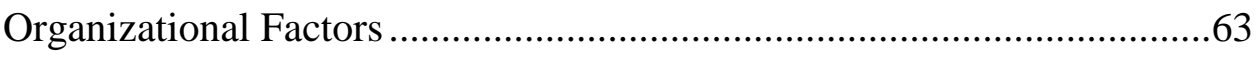

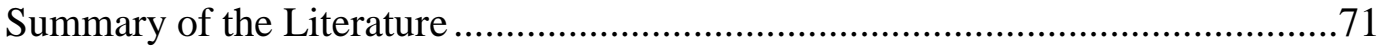

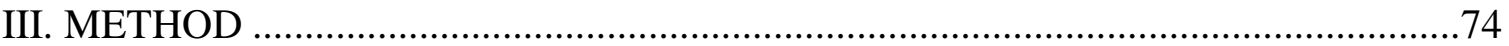

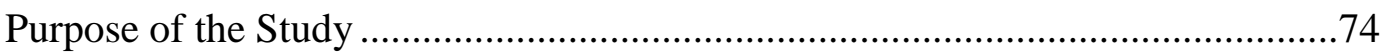

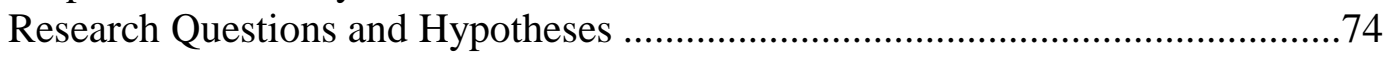

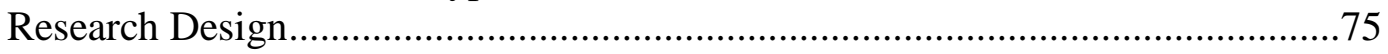

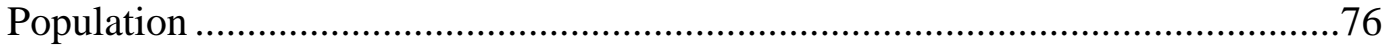

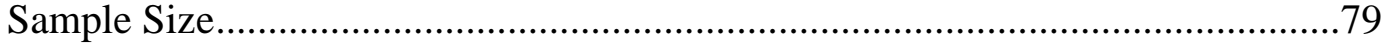

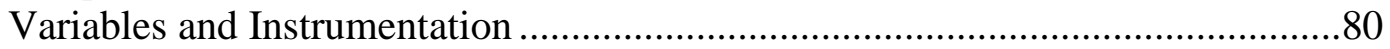

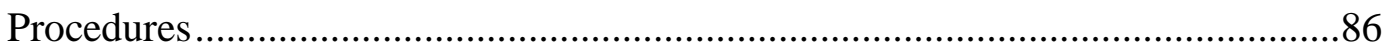


Behavior of Research Participants on MTurk........................................86

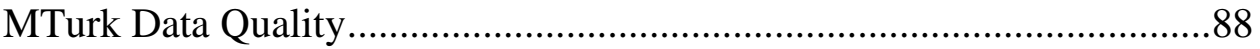

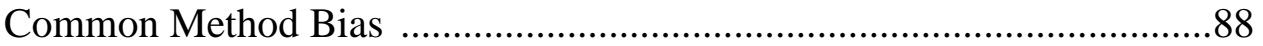

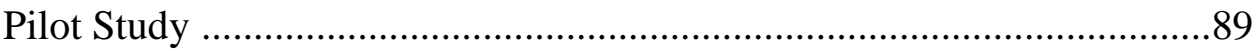

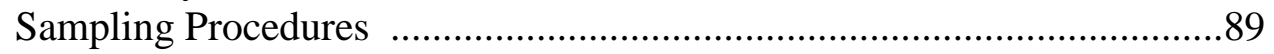

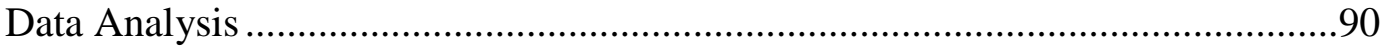

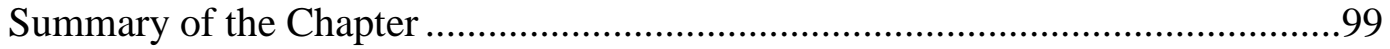

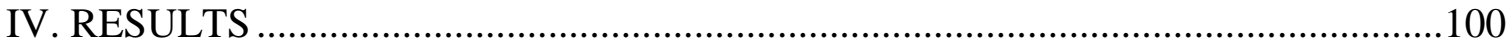

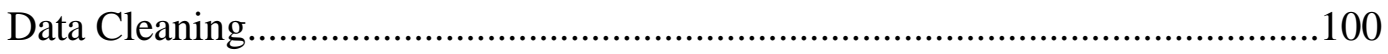

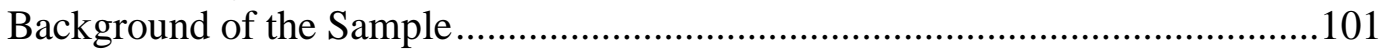

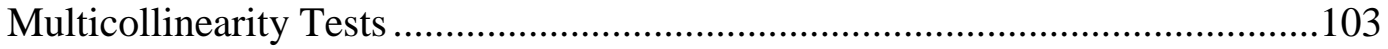

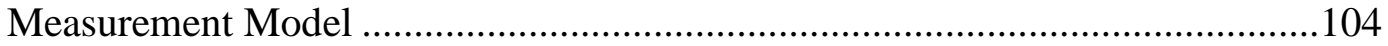

Multivariate Normality Tests ..........................................................105

Construct Reliability Tests..............................................................106

Convergent and Discriminant Validity Tests......................................108

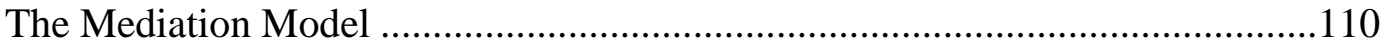

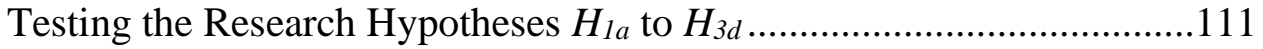

Additional Findings .........................................................................113

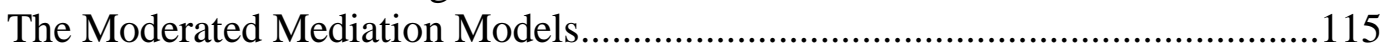

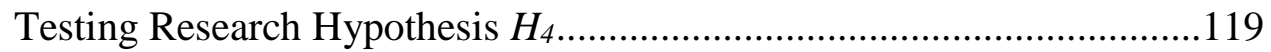

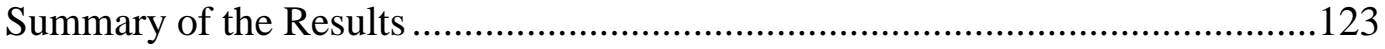

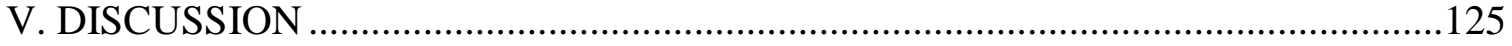

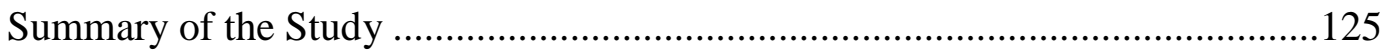

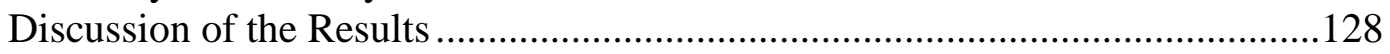

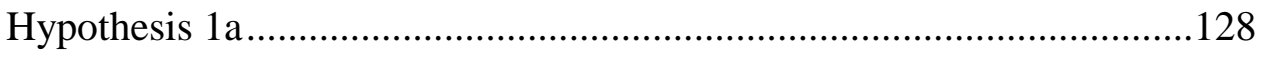

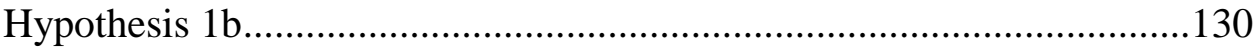

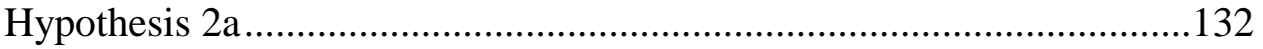

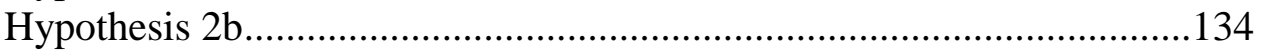

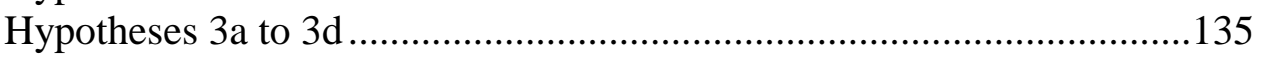

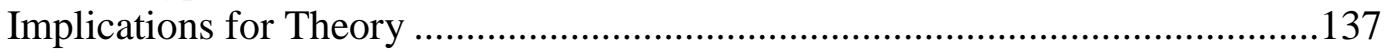

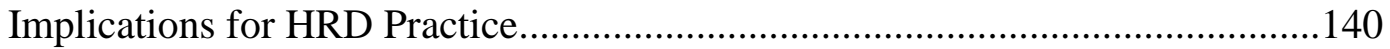

Limitations and Recommendations for Future HRD Research ........................142

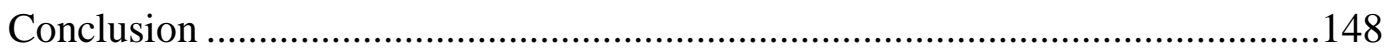

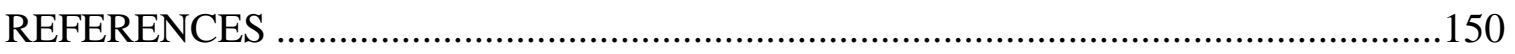

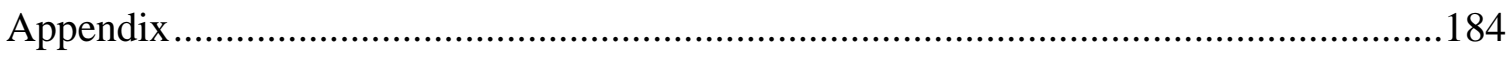

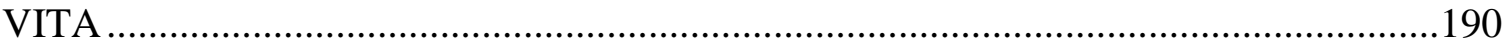




\section{LIST OF TABLES}

TABLE

PAGE

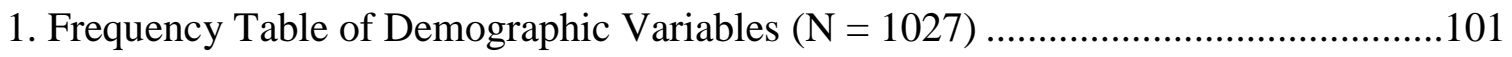

2. Zero-Order Correlations among the Research Variables.........................................104

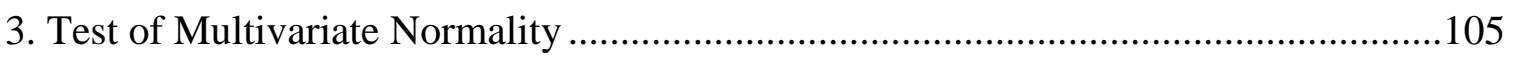

4. Standardized Loadings and Reliability Coefficients for Measurement Model............106

5. Average Variance Extracted and Interconstruct Squared Correlations ......................108

6. Bootstrapped Standardized Path Coefficients for Structural Model ..........................111

7. Model Fit Statistics for Low and High Workgroup Civility Climate Models .............116

8. Bootstrapped Standardized Path Coefficients for Moderator Groups .......................116

9. Moderation of Workgroup Civility Climate on Path Strengths ................................122

10. Specific Indirect Effects for Low Workgroup Civility Climate Model ....................122 


\section{LIST OF FIGURES}

FIGURE

PAGE

1. Hypothesized mediation model of instigated incivility ........................................19

2. Measurement model with standardized coefficients ............................................109

3. Mediation model with standardized coefficients ...............................................114

4. Low workgroup civility climate model with standardized coefficients.....................117

5. High workgroup civility climate model with standardized coefficients ....................118

6. Significant path differences between moderator groups.......................................121

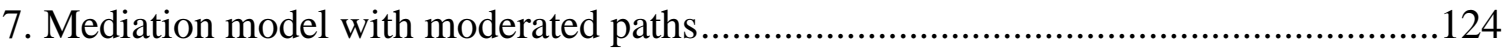




\section{CHAPTER I}

\section{INTRODUCTION}

The present study develops and tests a parallel multiple mediator model of instigated incivility moderated by workgroup civility climate. The first part of this chapter includes the background to the problem, followed by the problem statement, purpose of the study, research questions and hypotheses, and theoretical framework. The second part of Chapter I includes the hypothesized model, the significance of the study, assumptions of the study, delimitations of the study, definition of terms, and concludes with the organization of the study.

\section{Background of the Problem}

Uncivil behavior in the workplace has increased in recent years (Cortina, KabatFarr, Leskinen, Huerta, \& Magley, 2017; Estes \& Wang, 2008; Lim, Cortina, \& Magley, 2008; Pearson, Andersson, \& Porath, 2005; Porath \& Pearson, 2013; Pearson \& Porath, 2009; Reio \& Ghosh, 2009). In an American study, 54\% of participants had instigated incivility towards coworkers in the past year, $12 \%$ had engaged in uncivil behavior several times, and 3\% had done so on a daily basis (Reio \& Ghosh, 2009). Workplace incivility is defined as "low intensity deviant behavior with ambiguous intent to harm the target, [that is] in violation of workplace norms for mutual respect" (Andersson \& Pearson, 1999, p. 457). Examples of workplace incivility include making condescending or demeaning comments, using inappropriate language, ignoring and excluding coworkers, invading the privacy of others, and gossiping (Blau \& Andersson, 2005; Cortina \& Magley, 2009; Gray, Carter, \& Sears, 2017; Lim \& Cortina, 2005). Workplace 
incivility is a precursor to more serious negative outcomes that include physical violence (Andersson \& Pearson, 1999; Torkelson, Holm, Bäckström, \& Schad, 2016).

Uncivil behavior in the workplace poses a challenge for human resource development (HRD) professionals because of the potential significant negative outcomes for individuals, teams/groups and organizations (Bunk \& Magley, 2013; Cortina et al., 2017; Reio \& Ghosh, 2009). Over 1,700 workplace incivility articles published between 2011 and 2015 document the effects of workplace incivility (Cortina et al., 2017). Uncivil behavior affects individuals who experience incivility (targets; Porath \& Pearson, 2013), instigate incivility (perpetrators; Reio \& Ghosh, 2009), as well as individuals who observe uncivil behaviors (onlookers; Reich \& Hershcovis, 2015). Targets of workplace incivility experience negative consequences in (a) affect/job attitudes (e.g., emotional exhaustion, negative affect, career salience, job satisfaction, and organizational commitment), (b) cognition (e.g., perceived fairness and memory recall), and (c) behaviors (e.g., retaliatory incivility, task performance, work engagement, creativity and citizenship behavior; Cortina et al., 2017; Schilpzand, De Pater, \& Erez, 2016). Similar negative outcomes of workplace incivility are surfacing among individuals who witness incidents of workplace incivility (Chui \& Dietz, 2014; Reich \& Hershcovis, 2015; Totterdell, Hershcovis, Niven, Reich, \& Stride, 2012). Less is known about the consequences of workplace incivility on the perpetrators themselves (Cortina et al., 2017; Schilpzand et al., 2016; Trudel \& Reio, 2011). Scott, Restubog, and Zagenczyk, (2013) found that coworkers distrust perpetrators of incivility and that perpetrators suffer greater exclusion from workplace relationships. 
Subtleness and ambiguity distinguish incivility from other forms of interpersonal mistreatment (Lim et al., 2008), thus creating a problem for both the victims of incivility and their organizations (Estes \& Wang, 2008; Lim \& Lee, 2011). Given its subtle nature, uncivil behavior often goes unnoticed by management (Kabat-Farr \& Cortina, 2012). Ambiguous and low intensity behaviors are verbal rather than physical, passive versus active, indirect versus direct, and covert instead of overt (Andersson \& Pearson, 1999; Cortina, Kabat-Farr, Leskinen, Huerta, \& Magley, 2013; Pearson \& Porath, 2009). Such subtle behaviors as ignoring, interrupting, or excluding individuals from professional activities fall within the conceptualization of incivility because of their ambiguity with respect to harm (Kabat-Farr \& Cortina, 2012). The subtle nature of workplace incivility often bypasses formal anti-bullying rules, sexual harassment, and discrimination laws (Binggeli, Krings, \& Sczesny, 2014; Brownridge \& Halli, 2002; Fox \& Stallworth, 2005; Montgomery, Kane, \& Vance, 2004; Power et al., 2013; Trudel \& Reio, 2011).

A comprehensive review of workplace incivility literature conducted by Schilpzand et al. (2016) documented the incidence of perceived incivility in various industries. Workplace incivility has been studied in a wide variety of industries that include: (a) government (Cortina, Magley, Williams, \& Langhout, 2001; Cortina et al., 2002; Miner-Rubino \& Cortina, 2004), (b) banking (Lim \& Teo, 2009; Sliter, Jex, Wolford, \& McInnerney, 2010; Sliter, Sliter, Withrow, \& Jex, 2012), (c) manufacturing (Wu, Zhang, Chiu, \& He, 2013), (d) healthcare (Blau, 2007; Leiter, Lashinger, Day, \& Oore, 2011; Leiter, Price, \& Spence Lashinger, 2010; Oore et al., 2010; Spence Laschinger, Leiter, Day, \& Gilin, 2009; Spence Laschinger, Leiter, Day, Gilin-Oore, \& Mackinnon, 2012; Trudel \& Reio, 2011), education (Cortina \& Magley, 2009; Sakurai \& 
Jex, 2012), (e) customer service (Deifendorff \& Croyle, 2008; Kern \& Grandey, 2009; Scott et al., 2013; Walsh, Magley, Reeves, Davies-Schrils, Marmet, \& Gallus, 2012), and (f) engineering (Adams \& Webster, 2013).

An interesting dimension of incivility is its frequent association with positions of power (Callahan, 2011; Harcourt, 2012). Substantial evidence ties uncivil workplace behavior to the power position of the instigator, framing the act of incivility as a way of exercising power (Cortina et al., 2001; Estes \& Wang, 2008; Porath, Overbeck, \& Pearson, 2008). The sociologist Norbert Elias (1978) traced the interconnection between the evolution of manners and social hierarchies (e.g., class rank, social status, and political standing), establishing a solid discourse about interconnected nature of power and the enforcement of norms of civil conduct. The relationship between hierarchical systems and power is well documented and influences both the attitudes and behaviors of individuals (Kteily, Sidanius, \& Levin, 2011; Ho et al., 2015; Magee \& Galinsky, 2008; Pratto \& Stewart, 2012; Pratto et al., 1994; Sidanius \& Pratto, 1999). Similarly, workplace incivility scholars have noted that hierarchical differentials positively relate to uncivil workplace behavior (Caza \& Cortina, 2007; Cortina et al., 2002; Pearson et al., 2000). Perpetrator characteristics such as gender (Cortina et al., 2001; Crampton \& Hodge, 2008; Gallus, Bunk, Matthews, Barnes-Farrell, \& Magley, 2014; Pearson et al., 2000; Reio \& Ghosh, 2009), and higher positions of power within the organization (Cortina et al., 2001; Meier \& Gross, 2015; Trudel \& Reio, 2011) influence uncivil workplace behaviors. Other scholars have identified organizational factors that influence workplace incivility. Foulk, Woolum, and Erez (2016) state that "catching rudeness is like catching a cold" (p. 50), suggesting that a generalized workplace climate of rudeness 
has an impact on instigated incivility. For example, witnessing incivility in the workplace is linked to incivility perpetration (Holm, Torkelson, \& Bäckström, 2015). Aggressive behaviors instigated by coworkers are significant predictors of individual acts of uncivil behavior after controlling for variables such as gender, tenure, and individual differences (Glomb \& Liao, 2003). Thus, workplace civility norms are a significant factor in how uncivil behavior unfolds within organizations (Andersson \& Pearson, 2005; Walsh et al., 2012; Walsh, Lee, Jensen, McGonagle, \& Samnani, 2018).

Uncivil workplace behavior occurs with less frequency within organizations that support norms for civility that encourage employees to behave in a respectful manner (Gill \& Sypher, 2009; Leiter et al., 2012; Walsh et al., 2012, 2018). Workgroup civility climate impacts how employees interpret the behaviors they observe in the workplace (Walsh et al., 2012, 2018). Civility climate refers to "employee perceptions of norms supporting respectful treatment among workgroup members" (Walsh et al., 2012, p. 8). Organizations that address uncivil behavior and encourage the modeling of respectful behavior create a civility climate that promotes civil employee behavior consistent with the workplace civility norms of the organization (Walsh et al., 2012,2018). Montgomery and colleagues (2004) found that demographic characteristics predicted group level norms of civility, and noted differences in observer perceptions of norm violations depending on the how the observer identifies with the instigator and victim of incivility. The way people assess uncivil workplace behavior may be linked to larger social forces that guide behavioral expectations (Miner et al., 2018).

Cortina et al. (2002) suggested a link between workplace incivility and social dominance, as represented by social dominance theory. Social dominance theory (SDT) 
contends that all societies are structured as systems of group-defined social hierarchies with dominant groups at the top and subordinate groups at the bottom (Sidanius \& Pratto, 1993). Social dominance theory postulates that societies create ideologies that promote the superiority of one group over another to promote and maintain group inequality (Pratto, Sidanius, Stallworth, \& Malle, 1994). To understand the factors that support or reject ideologies that promote inequality, Pratto et al. (1994) introduced an individual difference variable called Social Dominance Orientation (SDO) to measure the extent that an individual desires to dominate and be superior to a group perceived by the individual as being composed of inferior members. Scholars have identified SDO as a strong predictor of prejudice (Duckitt \& Sibley, 2007; Ho et al., 2015; Ho et al., 2012; Pratto, Sidanius, \& Levin, 2006; Pratto et al., 1994; Pratto, Stewart, \& Zeineddine, 2013). In organizational studies, SDO is linked to instigated incivility (Daniels, Simmons, \& Hall 2015), workplace bullying (Parkins, Fishbein, \& Ritchey, 2006), abusive supervision (Hu \& Liu, 2017), and interpersonal power dynamics in organizational settings (Aiello, Tesi, Pratto, \& Pierro, 2018). Current evidence suggests that the construct of SDO includes two specific dimensions of social dominance (Ho et al., 2012, 2015). One dimension addresses the desire for intergroup dominance (SDO-D) and the other dimension subsumes the characteristics of intergroup antiegalitarianism (SDO-E; Ho et al., 2012, 2015). Desire for intergroup dominance (SDO-D) involves a preference for group-defined hierarchies where dominant groups actively oppress subordinate groups via overtly oppressive and aggressive behaviors (Ho et al., 2012, 2015). Intergroup antiegalitarianism (SDO-E) involves a preference for intergroup inequalities via a covert maintenance of subtle hierarchy-enhancing ideologies and social policies (Ho et al., 2012, 
2015). Social dominators are mostly likely to harbor feelings of racial prejudice and are more likely to be more sophisticated in expressing prejudiced feelings as well (Duriez \& Van Hiel, 2002). Thus, SDO values may be important for understanding employee inclinations to engage in deviant, counterproductive, or unethical workplace behaviors (Shao, Resick, \& Hargis, 2011).

In modern societies, racist attitudes are a social taboo and individuals are under pressure to manifest an egalitarian presentation of self even if prejudices are present (Githens, 2011). Incivility research posits that individuals harboring racist sentiments express their prejudice through less objectionable ways, such as ambiguous and low intensity forms of aggression toward a select group of individuals (Cortina, 2008). When social norms proscribe the expression of overt discrimination, a prejudiced individual may turn to incivility as a more socially acceptable way to exert their power over others (Githens, 2011). Cortina (2008) captured this emerging pattern by proposing a theory of selective incivility where individual characteristics are selective triggers to the incidences of incivility. Cortina (2008) posits that uncivil workplace behaviors may conceal racist sentiments. Being a racial or ethnic minority, being a female, and being younger in age, increase the odds of experiencing incivility (Binggeli et al., 2014; Brownridge \& Halli, 2002; Cortina et al., 2013; Cortina et al., 2017; Fox \& Stallworth, 2005; Leiter et al., 2010; Lim \& Lee, 2011; Montgomery et al., 2004; Power et al., 2013, Trudel \& Reio, 2011).

Most modern workplaces tend to promote an egalitarian ethos when it comes to racial or gender behavioral expectations (Aiello, Pratto, \& Pierro, 2013; Githens, 2011). Egalitarianism is a concept that rests on the principle that all human beings are equal in 
fundamental worth and moral status (Arneson, 2013). A non-egalitarian perspective, in contrast, involves the belief that individuals of certain races, ethnicities, or social statuses - either ascribed or achieved — have more moral worth than others (Dworkin, 1981). In the modern workplace, individuals manage feelings of prejudice to avoid sanctions and conflict (Githens, 2011).

Kim, Bhave, and Glomb (2013) theorize that working in a demographically diverse workgroup (in terms of age, race, and gender) leads to an increased need for individuals to regulate emotions. Demographic diversity may be a catalyst for emotion regulation in work environments because organizations generally require employees to hide personal biases (Kim et al., 2013). Emotion regulation refers to "the processes by which individuals influence which emotions they have, when they have them, and how they experience and express these emotions" (Gross, 1998b, p. 275). Employees can and do regulate their emotions to meet societal, occupational, and organizational norms (Grandy, 2000; Hochschild, 1983). Emotion regulation is known to be a critical skill for managing interpersonal conflict (Angie, Connelly, Waples, \& Kligyte, 2011; Hülsheger \&Schewe, 2011; Kammeyer-Mueller, Wanberg, Rubenstein, \& Song, 2013; Lench, Flores, \& Bench, 2011; Mesmer-Magnus, Glew, \& Viswesvaran, 2012).

In her analysis of the ways in which emotions are structured in the workplace, Hochschild (1983) uses the term "feeling rules" to describe organizational and societal norms about the appropriate type and amount of feeling that should be experienced in the workplace. According to Burkitt (2013), emotions orient us within a particular situation, as well as in relation to others who are part of that situation. The modal model of emotion posits that emotions involve person-situation transactions that require attention, have 
significance to an individual, and give rise to responses that may modify the personsituation transaction in critical ways (Barrett, Mesquita, Ochsner, \& Gross, 2007; Gross, 1998a; Gross \& Thompson, 2007). Empirical evidence demonstrates that individuals have relatively stable tendencies to employ emotion regulation strategies (Gross \& John, 2003; John \& Gross, 2004; Liu, Prati, Perrewé, \& Brymer, 2010). Individuals who employ the emotion regulation strategy of cognitive reappraisal intervene early in the emotion-generative process, which affects not only the behavioral display of emotion, but also what they feel inside (Gross \& John, 2003). In contrast, individuals who habitually resort to the emotion regulation strategy of expressive suppression intervene late in the emotion-generative process, are limited to what they express behaviorally, and thus mask their true feelings (Gross \& John, 2003). Incivility scholars have explored the role of emotion regulation within the context of experienced incivility from customers (Grandey, Dickter, \& Sin, 2004; Sliter et al., 2010). There is little insight in the incivility literature addressing the role of emotion regulation on instances of instigated incivility. Little is known about how interpersonal notions of power influence the way emotions are regulated in the workplace (Grandey, Deifendorff, \& Rupp, 2013) and subsequently how these may impact instigated incivility (Schilpzand et al., 2016).

\section{Problem Statement}

Workplace incivility is on the rise (Estes \& Wang, 2008; Lim et al., 2008;

Pearson et al., 2005; Pearson \& Porath, 2009; Pearson et al., 2000; Reio \& Ghosh, 2009), and the negative individual and organizational outcomes related to uncivil workplace behavior are well documented (Cortina et al., 2017; Hershcovis, 2007; Schilpzand et al., 2016). Workplace incivility is linked to negative individual outcomes such as depression 
(Lim \& Lee, 2011) increased levels of stress (Adams \& Webster, 2013; Cortina et al., 2001; Kern \& Grandey, 2009; Lim \& Cortina, 2005), emotional exhaustion (Kern \& Grandey, 2009; Sliter et al., 2010), and emotional labor (Adams \& Webster, 2013; Sliter et al., 2010). Workplace incivility corrodes organizational values and exhausts organizational resources (Porath \& Pearson, 2013). Incivility is linked to lower organizational commitment (Lim \& Teo, 2009), decreased task performance (Chen, Ferris, Kwan, Yan, Zhou, \& Hong, 2013; Sliter et al., 2012a), decreased work engagement (Chen et al., 2013), absenteeism (Sliter et al., 2012b), and turnover intentions (Lim et al., 2008; Wilson \& Holmvall, 2013).

Workplace incivility research focuses disproportionately on experienced incivility, leaving many questions unanswered about the instigators of incivility (Schilpzand et al., 2016). Incivility scholars uncovered numerous antecedents and outcomes of uncivil behavior that are related to experienced incivility - variables that explain incivility relationships from the target's perspective. Indeed, empirical research on workplace incivility in general is primarily studied through the perspective of the victim and few studies have explored the topic from the angle of the perpetrator (Jex, Geimer, Clark, Guidroz, \& Yugo, 2010; Liu, Chi, Friedman, \& Tsai, 2009; Meier \& Semmer, 2012; Schilpzand et al., 2016; Reio \& Ghosh, 2009). Workplace incivility studies mostly focus on the incidence and impact of incivility (e.g., Cortina et al., 2002; Cortina, 2008; Cortina et al., 2013; Vickers, 2006). Consequently, there is a considerable gap in the workplace incivility literature concerning instigated incivility, particularly its antecedents (Cortina et al., 2017; Jex et al., 2010; Liu et al., 2009; Meier \& Semmer, 2013; Reio \& Trudel, 2013; Schilpzand et al., 2016). One such under-researched 
antecedent variable that is linked to negative workplace behavior is social dominance orientation (Aiello et al., 2013; Daniels et al., 2015; Hu \& Liu, 2017; Shao, et al., 2011). Likewise, little research has examined possible mediator variables or variables that "carry" the relationship between a predictor variable on the outcome of workplace incivility (Schilpzand et al., 2016). One promising unexplored mediator of the relationship between social dominance orientation and uncivil behavior is linked to how employees regulate emotions (Hodson \& Costello, 2007; Matthews \& Levin, 2012; Van Hiel, Duriez, \& Kossowska, 2006). Employee emotions are identified as leverage points for HRD practitioners to reduce instigated incivility within organizations (Reio \& Ghosh, 2009). Workgroup civility climate norms (Cortina, Rabelo, \& Holland, 2018; Walsh et al., 2012, 2018) and emotion regulation strategies may explain the trajectory of workplace behaviors (Grandey, 2000; Grandey \& Gabriel, 2015). Instigated incivility research might yield new insights into the implementation of policies and practices to reduce its likelihood in the workplace (Cortina et al., 2018).

\section{Purpose of the Study}

The purpose of this study was to develop and test a parallel mediator model of instigated incivility moderated by workgroup civility climate. The present research examined the mediation of emotion regulation strategies of cognitive reappraisal and expressive suppression, on two types of social dominance orientation - support for intergroup dominance (SDO-D) and intergroup antiegalitarianism (SDO-E) - on the outcome of instigated incivility, and tested the moderating effects of workgroup civility climate on the paths of the hypothesized mediation model. 


\section{Research Questions and Hypotheses}

Two research questions and four major hypotheses guided this study.

Research question 1: Do the emotion regulation strategies of cognitive reappraisal and expressive suppression mediate the relationship between the two types of social dominance orientation (SDO-D and SDO-E)? The following hypotheses were tested:

Hypothesis 1a: There is a statistically significant positive relationship between SDO-D and instigated incivility.

Hypothesis $1 b$ : There is a statistically significant positive relationship between SDO-E and instigated incivility.

Hypothesis 2a: There is a statistically significant relationship between SDO-D and the mediators expressive suppression and cognitive reappraisal.

Hypothesis $_{2 b}$ : There is a statistically significant relationship between SDO-E and the mediators expressive suppression and cognitive reappraisal.

Hypothesis 3a: Expressive suppression mediates the relationship between SDO-D and instigated incivility, while controlling for SDO-E.

Hypothesis 36 : Cognitive reappraisal mediates the relationship between SDO-D and instigated incivility, while controlling for SDO-E.

Hypothesis $3 c$ : Expressive suppression mediates the relationship between SDO-E and instigated incivility, while controlling for SDO-E.

Hypothesis 3 : Cognitive reappraisal mediates the relationship between SDO-E and instigated incivility, while controlling for SDO-D. 
Research question 2: What are the moderating effects of workgroup civility climate on the proposed model of instigated incivility? The following hypothesis was tested:

Hypothesis 4: The paths of the model are moderated by workgroup civility climate such that the strength or direction of one or more paths are significantly different for subsamples from different civility climates.

\section{Theoretical Framework}

The following section presents social dominance theory and the emotion regulation framework, which guided this research study on instigated incivility.

\section{Social Dominance Theory}

Prompted by the pervasive nature of group-based prejudice, Sidanius and Pratto (1999) developed social dominance theory (SDT). Social dominance theory is a systematic theory that operates at various levels of analysis to link individual personality traits and attitudes with institutional behavior and social structure (Sidanius \& Pratto). The theory postulates that to understand the creation and maintenance of social hierarchies, researchers need to connect the psychological and sociological processes giving rise to hierarchical social systems (Sidanius \& Pratto; Sidanius, Pratto, Van Laar, $\&$ Levin, 2004). The main assumption of the theory is that all human societies are structured as systems of group-based social hierarchies (Sidanius \& Pratto). At the very minimum, the hierarchical social structure consists of one or a small number of dominant groups at the top and one or several subordinate groups at the bottom (Sidanius \& Pratto). The dominant groups possesses a disproportionately larger share of social resources with positive social value in comparison to submissive groups (Sidanius \& Pratto). The theory 
distinguishes among three distinct stratification systems: age system, gender system, and arbitrary-set systems (e.g., socially constructed groups based on ethnicity, nationality, profession, and any other distinction that a culture can construct).

The establishment and maintenance of gender hierarchy is central to the theory of social dominance. Sidanius and Pratto (1999) suggest that social dominance might in part be an expression of evolved male psychology designed to compete with and ultimately exploit members of out-groups. The gender hierarchy is described as "fixed" because of evolved mating strategies, with men always at the top and women always lower than menon the hierarchy. Therefore, the theory proposes an "invariance hypothesis," which states that, all else being equal, women will always have lower levels of social dominance orientation (SDO) than men. There is evidence across several cultures indicating that women do typically score lower in measures of SDO than men (Bates \& Heaven, 2001; Pratto et al., 2006; Sidanius \& Pratto, 1999; 2012; Sugiura, Mifune, Tsuboi, \& Yokota, 2017).

While the gender-defined hierarchical system places females in the subordinate role and males in the dominant role across societies, the arbitrary-set hierarchy is established by more culture-specific constructions of domination (Sidanius \& Pratto, 1999, 2012). These arbitrary sets are the historically and culturally established systems of inequality often defined by ethnicity, race, class, tribe, nation, and other socially constructed divisions. Arbitrary sets are "different manifestations of the same basic human predisposition to form group-based social hierarchies" (Sidanius \& Pratto, 1999, pp. 37 -38). For Sidanius and Pratto (1999), most forms of prejudice and discrimination serve to produce and reflect a group-based hierarchy. 
One of the mechanisms which operates in society to maintain and promote groupbased hierarchies are the legitimizing myths (rationalizations, logics, beliefs, values, norms, rules, and ideologies), which produce moral and intellectual narratives justifying individual attitudes and behaviors (Pratto et al., 2006; Sidanius \& Pratto, 1999, 2012). Many elements of hierarchy-enhancing legitimizing myths are blatant acts of racism and sexism (Sidanius \& Pratto, 1999). While, subtle legitimizing myths come in the form of ideological constructs such as the notion of individual responsibility, the Protestant work ethic, internal attributions of misfortunes of the poor, and political conservatism (Sidanius \& Pratto, 1999, 2012; Tyler, 2005).

Sidanius and Pratto (1999, 2012) argue that groups establish and maintain social hierarchies through three processes: individual discrimination, institutional discrimination, and behavioral asymmetry. The first two processes include phenomena ranging from everyday biases, such as group-based favoritism in the labor and housing markets, to state-sanctioned use of force, such as ethnic/racial profiling, to the use of secret police forces and death squads. The third process, behavioral asymmetry, refers to the ways in which elite group members and subordinate group members differ culturally and systematically in their beliefs and patterns of behavior. The asymmetry, they argue, takes the shape of a symbiotic relationship between the dominant and subordinate group members. In other words, SDT frames oppression as a cooperative game, where members of subordinate categories not only tend to accept cultural beliefs, which demean them, but also often engage in self-debilitating behavior. For example, rejecting educational trajectories that would facilitate their own occupational success, or by endorsing self- 
defeating ideologies, such as a belief in the inherent superiority of the dominant group (Pratto et al., 2006).

Social dominance theory operates on the premise that individuals tend to create group-based social hierarchies that define a dominant and a subordinate group (Sidanius

\& Pratto, 1999). Hierarchies are defined by "social power, prestige, and privileges that an individual possesses by virtue of his or her ascribed group membership in a particular socially constructed group" (Sidanius \& Pratto, 1999, p. 32). Social dominance orientation (SDO), derived from social dominance theory, is one of the strongest predictors of racial and ethnic prejudice (Bergh, Sidanius, \& Sibley, 2015; Ho et al., 2012, 2015; Hindricks, Verkuyten, \& Coenders, 2014; Kteily, Bruneau, Waytz, \& Cotterill, 2015; Küpper, Wolf, \& Zick, 2010; Pratto et al., 1994; Sibley \& Duckitt, 2008). Organizational scholars have used social dominance theory to explore and explain negative workplace behaviors (Aiello et al., 2018; Daniels et al., 2015; Hu \& Liu, 2017; Parkins et al., 2006; Shao et al., 2011).

\section{Emotion Regulation Framework}

Efforts to operationalize a definition of emotion are impeded by the reality that the concept of emotion refers to an array of responses (Gross, 2014). The modal model of emotion posits that emotions involve person-situation transactions that require attention, have significance to an individual, and give rise to responses that may modify the personsituation relationship in critical ways (Barrett et al., 2007; Gross, 1998a; Gross \& Thompson, 2007). Meta-analyses have shown how emotions consistently impact cognitive and behavioral outcomes (Angie et al., 2011; Hülsheger \& Schewe, 2011; Kammeyer-Mueller et al., 2013; Lench et al., 2011; Mesmer-Magnus et al., 2012). 
Emotions can influence decision-making and affect interpersonal interactions (Fiske, 1998; Gross, 2014; Gross \& Thompson). What we experience and categorize as emotion can powerfully impact the way we perceive and engage with the world (Stefanucci, Gagnon, \& Lessard, 2011) and our emotional processes carry implicit information about the goodness and badness of the people and things around us (Clore \& Tamir, 2002). Emotions have a wide, sometimes unwelcomed influence on many aspects of the work environment (Barsade \& Gibson, 2012), and at times need to be regulated (Gross, 2013, 2014; Hochschild, 1983).

Emotion regulation refers to a broad set of activities considered to be emotional labor when meeting certain criteria (Grandey \& Melloy, 2017). Emotion regulation performed in the workplace to meet organizational display rules is called emotional labor, a term coined by Hochschild (1983) from her seminal work with flight attendants. Organizational display rules are behavioral standards established by the organization that indicate which emotional expressions are considered acceptable or not acceptable (Ekman, 1973). Emotional labor is distinct from emotion regulation (Gross, 1998b), in that emotional labor requires workers to manage feelings and expressions to help the organization profit (Grandey et al., 2013).

Grandey (2000) identified the need to go beyond the notion of emotional labor developed by Hochschild (1983). She suggests that specific strategies of emotion regulation are necessary to understand how employees manage their emotions at work. Grandey and Gabriel (2015) propose the linking of the emotional labor and emotional regulation literatures. Emotion regulation seeks to understand the processes that individuals use to manage their emotions (Gross, 1998b). Emotional labor, on the other 
hand, is only concerned with emotions as a means of meeting organizational display rules (Hochschild, 1983). The merging of the two organizational emotion research traditions open an opportunity for researchers to further explore emotions in the workplace (Grandey, 2000; Grandey \& Gabriel, 2015).

Hochschild (1983) conducted an extensive analysis of the structure of emotions in work environments. She argues that the work environment requires that employees express only emotions appropriate to their occupations and work settings. Hochschild uses the term "feeling rules" to describe organizational norms about the appropriate type and amount of feeling that should be experienced in a particular work situation. Emotion regulation (Gross, 1998b) refers to the actual strategies employees use to perform the emotion work that is required to accommodate the feeling rules that are established by the organization. The flight attendants in Hochschild's (1983) study, for example, understand that the emotional expectations of their job require them to suppress, rather than express, their emotions when confronted with unpleasant circumstances.

Research on emotion regulation mostly focuses on employee-customer interactions leaving emotion regulation in employee-employee interactions relatively unexplored (Grandey \& Gabriel, 2015; Kim et al., 2013). Employees may also regulate emotions to engage in impression management, or to establish social relationships when dealing with a supervisor or a coworker (Pearson et al., 2000). All jobs that involve interactions with people entail some degree of emotion regulation, as employees explicitly or implicitly follow emotional display rules, which are standards for the appropriate expression of emotions on the job (Diefendorff, Croyle, \& Gosserand, 2005). 
Organizational display rules and emotion regulation strategies may explain the trajectory of workplace behaviors (Grandey, 2000; Grandey \& Gabriel, 2015).

\section{Hypothesized Model of Instigated Incivility}

Building upon the aforementioned theoretical framework, the following section presents the hypothesized model of instigated incivility and an overview of the variables included in this study. First, the hypothesized model of instigated incivility is presented (Figure 1). Second, the variables included in the study are discussed in the following order: (a) predictor variables - SDO-D and SDO-E, (b) mediator variables - cognitive reappraisal and expressive suppression, (c) outcome variable - instigated incivility, and (d) the moderator variable - workgroup civility climate.

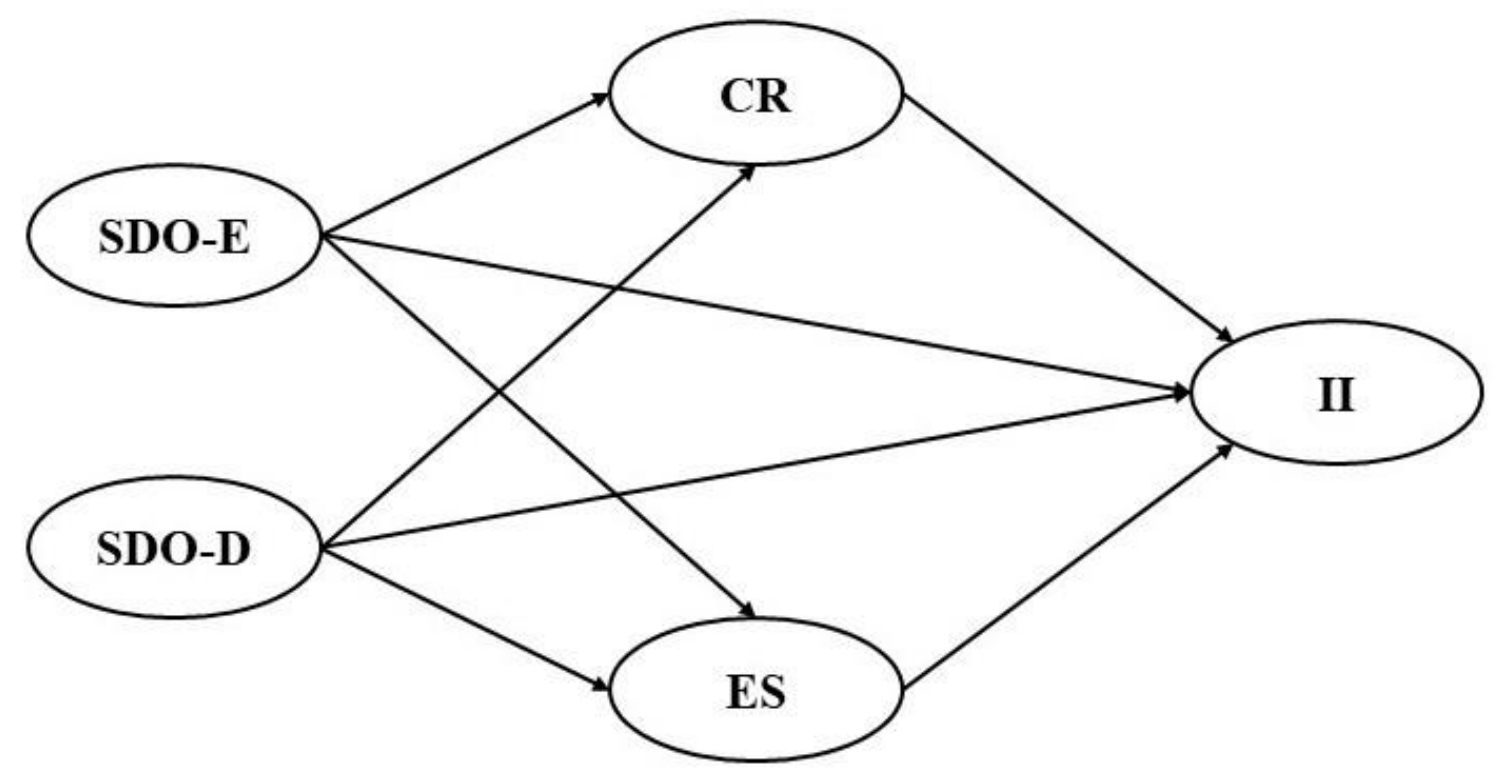

Figure 1. Hypothesized mediation model of instigated incivility. Note. SDO-D = Intergroup Dominance; SDO-E = Intergroup Antiegalitarianism; CR = Cognitive Reappraisal; ES = Expressive Suppression; II = Instigated Incivility. 


\section{Predictor Variables - SDO-D and SDO-E}

It was hypothesized that the two dimensions of social dominance (SDO-D and SDO-E) predict instigated incivility. Social dominance scholars indicate that the two dimensions of SDO are related, but that the two dimensions predict different forms of behavior (Bergh et al., 2015; Ho et al., 2012, 2015). Intergroup dominance (SDO-D) is an empirically sound measure related to overt racism and aggression directed toward groups that dominant individuals desire to oppress (Ho et al, 2012; Ho et al., 2015). Intergroup dominance is linked to aggression and violence toward outgroups, overt forms of racism, blatant forms of dehumanization, and sexism (Bergh et al., 2015; Ho et al., 2012; Ho et al., 2015; Hindriks et al., 2014; Kiteily et al., 2015).

Intergroup antiegalitarianism (SDO-E), is associated with a disposition to pursue covert and passive antiegalitarian ideologies with the aim of limiting equitable access to resources and social power among lower power groups (Ho et al., 2015). Intergroup antiegalitarianism is linked to non-inclusive and nonegalitarian preferences regarding intergroup relations and best predicts subtle hierarchy-enhancing ideologies and covert forms of prejudice (Ho et al., 2012). Social dominance scholars have linked SDO-E to political conservativism, support for unequal distribution of resources, and opposition to policies that address equality (Bergh et al., 2015; Ho et al., 2015). Subtle and covert forms of prejudice are outcomes of strong SDO-E traits (Bergh et al., 2015; Hindriks et al., 2014; Ho et al., 2012; Ho et al., 2015; Jost \& Thompson, 2000; Kiteily et al., 2015; Kugler, Cooper, \& Nosek, 2010). 


\section{Mediator Variables - Cognitive Reappraisal and Expressive Suppression}

Employees who harbor prejudice are expected to regulate emotions in the workplace to adhere to societal and organizational expectations (Githens, 2011). The hypothesized model of instigated incivility suggests that the emotion regulation strategies of cognitive reappraisal and expressive suppression mediate the relationship between the two types of social dominance (SDO-D and SDO-E) and instigated incivility. Variables function as mediators to the degree that they account for the relation between the predictor variable and the outcome variable, thus mediators address how or why certain effects occur (Baron \& Kenny, 1986).

The emotion regulation strategy of cognitive reappraisal involves construing a potentially emotion-eliciting situation in a way that changes its emotional impact (Gross \& John, 2003; Hochschild, 1983; Webb, Miles, \& Sheeran, 2012). The strategy of cognitive reappraisal influences whether or not particular emotion response tendencies are triggered and are therefore expected to have generally positive implications for the avoidance of conflict and aggression (Gross \& Thompson, 2007; Grandey, 2000; Gross, 1998b; Hochschild, 1983; Liu et al., 2010; Webb et al., 2012). Reappraisal allows for changes in both the internal emotional experience and the emotional expression of that experience (Gross, 2002; Koole, 2009). In the emotional labor literature, individuals use cognitive reappraisal in the attempt to modify feelings to match the required display rules of the organization (Grandey \& Daniels, 2015). Individuals try to align both the required and the true feelings when they engage in this form of emotion regulation. The use of the emotion regulation strategy of cognitive reappraisal is empirically linked to adaptive 
emotional and social outcomes (Denny \& Ochsner, 2014; Gross \& John, 2003; Troy, Wilhelm, Shallcross, \& Mauss, 2010).

In contrast to cognitive reappraisal, the expressive suppression of emotion modulates emotion response tendencies once they have been triggered and are therefore expected to have generally more negative implications for the expression of aggression (Gross, 1998b; Gross \& Levenson, 1993; Hochschild, 1983). Individuals engaging in emotion suppression are putting on a mask by suppressing, amplifying, or faking emotions (Hochschild, 1983; Grandey \& Daniels, 2015). Expressive supression entails experiencing emotional dissonance because feelings and actions diverge (Hochschild, 1983). Researchers identified that employees who report regularly having to display emotions that conflict with their own feelings are more likely than others to experience emotional exhaustion (Van Dijk \& Brown, 2006; Glomb \& Tews, 2004; Zapf \& Holz, 2006). Furthermore, emotion suppression is linked to impaired memory, greater experience of negative emotions, lessened social support, increased avoidant behaviors, and more depressive symptoms in comparison with the emotion regulation strategy of cognitive reappraisal (Gross, 2002; Gross \& John, 2003; John \& Gross, 2004). Finally, emotion suppression only affects emotional expression and does not change the emotional experience (Gross, 2002; Koole, 2009).

\section{Outcome Variable - Instigated Incivility}

Instigated incivility includes behaviors such as making condescending or demeaning remarks, using inappropriate language or tone, excluding coworkers from workplace activities, invading privacy, and gossiping (Blau \& Andersson, 2005; Cortina \& Magley, 2009; Gray et al., 2017; Martin \& Hine, 2005; Schilpzand et al., 2016). The 
recent development of a new Uncivil Workplace Behavior Questionnaire (UWBQ-I;

Gray et al., 2017) includes four dimensions of instigated incivility: (a) hostility, (b)

privacy invasion, (c) exclusionary behavior, and (d) gossiping.

\section{Moderator Variable - Workgroup Civility Climate}

Given the importance of workplace norms on the outcome of workplace incivility (Cortina et al., 2017; Schilpzand et al., 2016), the present study included workgroup civility climate as a moderator of the relationships specified by the hypothesized model of instigated incivility. Moderators are variables that affect the direction and or the strength of the relation between a predictor and outcome variable, which implies that the relation between the variables changes as a function of the moderator variable (Baron \& Kenny, 1986). Andersson and Pearson's (1999) introduced the construct of workplace incivility and included a reference to workplace norms to define workplace incivility uncivil behavior is "in violation of workplace norms for mutual respect" (p. 457). Workgroup civility climate norms are known to impact the level of uncivil behavior that occurs within organizations (Andersson \& Pearson, 2005; Cortina et al., 2017; Foulk, Woolum, \& Erez, 2016; Gallus et al., 2014; Gill \& Sypher, 2009; Leiter et al., 2012; Schilpzand et al., 2016; Walsh et al., 2012). Walsh and colleagues (2012) define workgroup civility climate norms as the perceptions that employees report concerning the respectful treatment of members within the workgroup.

\section{Significance of the Study}

Workplace incivility research is important for the field of HRD as the primary role of the field is to create and support heathy and productive work environments (Callahan, 2011; Estes \& Wang, 2008; Githens, 2011; Reio \& Sanders-Reio, 2011; 
Trudel \& Reio, 2011). There is a need to identify patterns that inform the process of instigated incivility to reduce its incidence and impact (Cortina, et al., 2018; Brunetta \& Reio, 2016; Jex et al., 2010; Meier \& Semmer, 2012; Schilpzand et al., 2016). The current study was the first to examine the relationship between the emotion regulation strategies of cognitive reappraisal and expressive suppression on two types of socially dominant employees (SDO-D and SDO-E), to evaluate how these emotion regulation strategies impact uncivil workplace behavior instigated by socially dominant employees, and to test these relationships in uncivil and civil workgroup civility climates. Findings from the study advance HRD scholarship and practice by offering knowledge that may help organizations minimize the incidence and impact of workplace incivility. Exploring emotion regulation strategies as variables that may explain how instigated incivility unfolds when employees have strong social dominance orientations could serve as a structure for developing more effective HRD strategies to address civility initiatives within diverse organizations. Emotional regulation strategies in the process of prejudice expression seem to be an under-studied, but important phenomenon in intergroup relations. Organizational researchers identified that high levels of SDO predict instigated incivility, interpersonal deviance, abusive supervision, and the use of harsh power tactics (Aiello et al., 2018; Daniels et al., 2015; Hu \& Liu, 2017; Shao et al., 2011), however there is a gap in the workplace literature on how the specific dimensions of SDO influence organizational behavior.

\section{Assumptions of the Study}

Because Schwarz (1999) argues that respondents tend to answer survey questions honestly and to the best of their ability, as predicted by communication theory, the 
researcher assumed that participants the present study did their best to participate with the researcher by answering the survey questions honestly and correctly. Cooperation is particularly important in the present study because the scales used inquired about psychological responses and behaviors that some individuals might not want to reveal.

A key conceptual assumption of the study was that the social category of "workers" has an existential cohesion, which allows for generalizability across worksites. The study assumed that all workers can be placed somewhere on the social dominance spectrum, regardless of the specific workplace environment, which is worthy of study to explore the process of instigated incivility.

A final assumption was that the Mechanical Turk (MTurk) research platform is robust enough to allow for the use of the data gathered in the testing of the proposed model of instigated incivility. Samples drawn from MTurk are comparable to samples selected by more conventional methods and superior to student samples in their generalizability (Buhrmaster, Kwang, \& Gosling, 2011; Mason \& Suri, 2012; Paolacci et al., 2010).

\section{Delimitations of the Study}

No study can claim to explore all the complexities associated with even the simplest of human behavior. To explore the psychosocial forces influencing the behavior of instigated incivility, the current study employed specific conceptual and empirical boundaries. These delimitations relate strongly to the theoretical framework, empirical measurement decisions of theoretical concepts, and research questions that guided the research. The present study explores the relationship between the theoretical constructs of social dominance orientation, the emotion regulation strategies of cognitive reappraisal 
and expressive suppression, and instigated incivility. The relationships among these concepts are based on the status of the respondent as "workers." While the respondents are all workers, they do not work in the same workplace. The potential effects of specific workplace environments might have an impact on the relationships among the explored variables, but that impact was not examined in this study.

The decision to use the Likert scales established in the literature was made to contribute to the literature using these measures, but also to expand it by exploring new relationships between the measured concepts. At the same time, the Likert instrument might be accused of simplifying the complexities working in the real world that give rise to uncivil behavior. The risks associated with using Likert instruments are worth taking to explore the possible relationships among the guiding concepts: SDO-D and SDO-E, the emotion regulation strategies of cognitive reappraisal and expressive suppression, and instigated incivility.

\section{Limitations of the Study}

While social scientific research utilizing the MTurk platform is expanding and gaining acceptance, there are certain limitations to its effectiveness. The non-random nature of the sample limits its generalizability because it may be unrepresentative of specific types of populations.

Another limitation of the study is the type of respondents selected for this study. It is well known that a variety of factors influence the dynamics of the work place (e.g., physical environment, work culture, type of workforce). As mentioned in the delimitations, the researcher has chosen to study "workers" as an operational concept. The current research is not studying a specific worksite or type of worksite. The study 
does not take into consideration the variety of worksite factors that might have an impact on an individual's behavior. The focus of the research is on how socially dominant individuals regulate emotions in relation to socially established norms of civility. While it may be possible to generalize the findings to the social category of "workers," the researcher is not able to speak about workers in specific industries (e.g., service sector, industrial sector) with as much confidence.

Conceptually, a limitation of the study is the lack of concern with the origins of the social dominance perspective of the respondents. Respondent demographic data were collected, but will not be used to explore the causal processes leading to the creation of a specific social dominance profile.

Another limitation is common method bias (CMB), which is a potential source of measurement error that poses a threat to the relationships found between measures of different constructs (Reio, 2010). Common method bias occurs when the relationship between variables is inflated or deflated as a result of the use of self-report measures (Podsakoff, MacKenzie, Lee, \& Podsakoff, 2003; Richardson, Simmering, \& Sturman, 2009; Spector, 2006). To minimize CMB this study followed the procedures recommended by Reio (2010) and Podsakoff et al (2003).

Finally, the cross-sectional nature of the present study limits its ability to establish causality between the two dimensions of social dominance orientation, emotion regulation strategies, and instigated incivility. The scope of the study is to identify "causal" paths that can be explored by future experimental or longitudinal research methodology. 


\section{Definition of Terms}

Abusive supervision is defined as "Subordinates' perceptions of the extent to which supervisors engage in the sustained display of hostile verbal and nonverbal behaviors, excluding physical contact.” (Tepper, 2000, p. 178).

Arbitrary-set systems are socially constructed groups defined by ethnicity, nationality, profession, and any other distinction that a culture creates (Sidanius \& Pratto, 1999).

Bullying is defined as "Repeated and enduring aggressive behaviors that are intended to be hostile and/or perceived as hostile by the recipient." (Einarsen, 1999, p. 131).

Cognitive reappraisal is defined as the attempt to reinterpret an emotion-eliciting situation in a way that alters its meaning and changes its emotional impact (Gross \& John, 2003; Lazarus \& Alfert, 1964).

Egalitarianism is a concept that rests on the principle that all human beings are equal in fundamental worth and moral status (Arneson, 2013).

Emotion regulation refers to the process by which we influence which emotions we have, when we have them, and how we experience and express them (Gross, 1998b).

Expressive suppression is defined as the attempt to hide, inhibit or reduce ongoing emotion-expressive behavior (Gross \& Levenson, 1993; Gross \& John, 2003).

Hierarchical systems are defined by "social power, prestige, and privileges that an individual possesses by virtue of his or her ascribed group membership in a particular socially constructed group" (Sidanius \& Pratto, 1999, p. 32). 
Interpersonal refers to patterns of behavior encountered when individuals interact or "connect" with one another (Goleman, 2006).

Selective incivility is defined a veiled form of sexism and racism where women and minority groups are selectively targeted with expressions of uncivil behavior (Cortina, 2008).

Social dominance orientation is "the extent to which one desires that one's ingroup dominate and be superior to outgroups" (Pratto et al., 1994, p. 742).

Supervisors are defined as "those who possess a formal status in a given hierarchy, hold higher ranking positions than their subordinates, and are in charge of assigning and organizing duties" (Abas \& Otto, 2016, p.7).

Worker is defined to include "an individual who has entered into or works under a contract of employment" (Davidov, 2005, p. 57).

Workgroup civility climate is defined as "employee perceptions of norms supporting respectful treatment among workgroup members" (Walsh et al., 2012, p. 8).

Workplace Incivility is defined as a "low intensity deviant behavior with ambiguous intent to harm the target, in violation of workplace norms for mutual respect" (Andersson \& Pearson, 1999, p.457).

\section{Organization of the Study}

Chapter I included an overview of the study. Chapter II presents a review of the literature to support the study. Chapter III describes the research methods of this study. Chapter IV presents the results of the data analysis. Chapter V concludes this study with a discussion of the results, implications for theory and practice, and limitations and recommendations for future research. 


\section{CHAPTER II}

\section{LITERATURE REVIEW}

Chapter II begins with an introduction to social dominance orientation (SDO) followed by literature addressing SDO in organizational studies, SDO on workplace behaviors, and individual characteristics of individuals with high SDO. Second, a review of the literature on the emotion regulation strategies of cognitive reappraisal and expressive suppression with a focus on affective and social outcomes is presented. Third, a review of the instigated incivility literature with an emphasis on individual and organizational factors that influence instigated incivility follows. The chapter concludes with the hypothesized model of instigated incivility and a summary.

\section{Social Dominance Orientation}

Sidanius and Pratto (1999) define SDO as "the degree to which individuals desire and support group-based hierarchy and the domination of 'inferior' groups by 'superior' groups" (p. 48). Research has validated the SDO construct as predictive of a multitude of intergroup attitudes and phenomena that contribute to the creation and maintenance of hierarchy between groups across a wide range of different samples, countries and contexts (Ho et al., 2015; Pratto et al., 1994; Sidanius \& Pratto, 1999; Vargas-Salfate, Paez, Liu, Pratto, \& Gil de Zúñiga, 2018). Individuals with high SDO tend to endorse ideologies that support the creation and maintenance of social hierarchies (e.g., racism and sexism), while those with a low SDO scores endorse narratives which attenuate social hierarchy (e.g., egalitarianism; Hiel \& Mervielde, 2002; Ho et al., 2012; Pratto et al., 1994). SDO predicts affective responses to novel situations and minimal groups, indicating that SDO is not limited to specific social groups, but extends to new social 
categories as well (Ho et al., 2015). Social dominators, regardless of social position, are likely to try to enhance their relative position by devaluing lower status groups (Kupper et al., 2010). There is considerable evidence indicating that SDO is one of the strongest predictors of prejudice against a wide range of denigrated groups, such as ethnic minorities, women, immigrants, poor people, and members of the LGBTQ community (Krupper et al., 2010; Kteily et al., 2011; Pratto et al., 1994; Sibley \& Duckitt, 2007). Social dominators are not only likely to be prejudiced, but their prejudice is likely to be more sophisticated (Duriez \& Van Hiel, 2002; Ho et al., 2015). High SDO scorers often exhibit a style of thinking which reflects a form of covert discrimination that surfaces in subtle ways when it is safe, socially acceptable, and easy to rationalize (Dovidio \& Gaertner, 1998; Ho et al., 2015). Further research identified the possibility that the original SDO scale is comprised of two distinct sub-dimensions of SDO (Jost \& Thompson, 2000; Kugler et al., 2010). These two dimensions of SDO, intergroup dominance (SDO-D) and intergroup antiegalitarianism (SDO-E) were empirically tested by Ho and colleagues in 2012. The following section first presents the literature on social dominance orientation (SDO) as unidimensional construct, followed by the literature on the two dimensions of social dominance SDO-D and SDO-E.

\section{Social Dominance Orientation as a- Unidimensional Construct}

Pratto et al. (1994) developed the first SDO measure to show that individual variation in SDO exists, and to demonstrate that SDO operates according to social dominance theory. Data collected from 13 college student samples included 1,952 participants. SDO strongly correlated with ethnic prejudice in all 13 samples with correlations ranging from .42 to .65 and averaging .55 . Ethnic prejudice was assessed 
with multiple measures including: McConahay's (1986) Modern Racism Scale and Katz and Hass' (1988) Pro-Black and Anti-Black Scale. The results were consistent with the notion that preference for group dominance drives specific forms of ethnic prejudice.

Kupper and collegues (2010) conducted a multi-country study measuring the relationship between SDO and attitudes toward immigrants in Europe. The study included samples of 1,000 participants representative of the population in eight European countries (Britain, France, Germany, Netherlands, Italy, Portugal, Poland, and Hungary). The countries surveyed contained a diverse range of immigrant populations and varying levels of social inequality. The authors tested the hypothesis that higher status groups discriminate more strongly against immigrants because high-status groups are more prone to SDO and adhere more closely to the legitimizing myths associated with hierarchy maintenance. Kupper et al. (2010) used a battery of items from well-established indices to measure anti-immigrant attitudes, diversity belief, discriminatory intent, and SDO. The results supported the hypotheses that individuals with higher SDO are more likely to harbor intent to discriminate against immigrants, partly because of their stronger antiimmigrant prejudice and partly because they are less likely to support diversity as a social good. Surprisingly, the results question the role of social status in determining SDO. Contrary to the relationship hypothesized by social dominance theory, individuals with lower socio-economic status were more prone to SDO and had stronger anti-immigrant attitudes along with weaker support for diversity. This surprising finding held even after the researchers controlled for the immigrant background of the respondent. Their evidence suggests that regardless of social position, individuals are likely to enhance their relative position by devaluing lower status groups (Kupper et al., 2010). 
In a study designed to explore the variables associated with social dominance orientation and authoritarianism, Duriez and Van Hiel (2002) documented a strong relationship between SDO and authoritarianism and an equally strong positive correlation between both variables and racism. Undergraduate Belgian students were asked to complete a questionnaire and to recruit neighbors to do the same. The resulting sample included 303 completed questionnaires (65\% females, 35\% males) checked for a variety of response biases and accepted as valid. The participants completed the Dutch version of the SDO scale, which included 14 items, a racism scale, and a cultural and economic conservatism scale. Not only did the authors find a strong relationship between SDO and prejudice, but they were able to distinguish between authoritarian-based prejudice and a dominance-based prejudice, which was more sophisticated, as it stems from a need to justify the maintenance of societal status inequalities (Duriez \& Van Hiel, 2002).

Organizational Studies. Social dominance theory posits that institutions obtain a fit between their members and the values of the institution, or a person-environment fit (P-E fit; Haley \& Sidanius, 2005; Sidanius \& Pratto, 1999). In other words, employers and employees tend to select one another to have compatible hierarchy-enhancing or hierarchy-attenuating beliefs. Haley and Sidanius (2015) explored the nexus of social position and ideology. They used vocational choice theory and social dominance theory as a framework to explore P-E fit. They built on research that posits that although workrelated values exist a priori to work itself, work-related values tend to match the values of eventual work environments. This P-E fit leads to superior job performance and higher employee satisfaction. They cite research conducted in hierarchy-enhancing organizations, such as police forces, showing that workers in these environments are 
higher in SDO while those working in hierarchy-attenuating organizations, such as civil liberties organizations, tend to be lower in SDO. This study focused on exploring the processes that create P-E fit at the worksite. The study identified five processes which merit further consideration: 1) self-selection (people select the types of jobs that fit their ideological [SDT] orientation, 2) institutional selection (organizations are inclined to select individuals who are ideological matches), 3) institutional socialization (once at the worksite, the individual's values and attitudes are shaped by rules, incentives, and peer pressures), 4) differential success (organizations invest in individuals with the right P-E fit and reward those with the right match), and 5) differential attrition (turnover occurs more frequently when there is a lack of congruence in the P-E fit). In their conclusions, the authors emphasize the need to investigate the interaction among these different processes and SDO to further develop social dominance theory within organizational contexts.

Kemmermeier, Danielson and Basten (2005) expanded the research on P-E fit by studying the relationship between political orientation and academic success. The authors posit, using social dominance theory, that academic disciplines that lead students into careers with access to social and economic power tend to favor students who have a strong commitment to upholding the existing social order. In a longitudinal study of 3,890 students at a major university in the United States, the researchers supported the hypothesis that student grades in hierarchy-enhancing disciplines (but not in hierarchyattenuating disciplines) correlate positively with a measure of conservatism scores measured at the start of the academic career. They did not use the SDO scale but analyzed the relationship between enrollments in high and low hierarchy-attenuating and 
hierarchy-enhancing courses, grades and political attitudes measured at the beginning of the university career. They concluded that hierarchy-enhancing environments (courses) within academia help reproduce the social hierarchy by bestowing rewards (higher grades) on individuals with conservative ideologies.

Workplace Behavior. Shao et al. (2011) explored the relationship between SDO and workplace interpersonal behaviors. The study included $(N=490)$ full-time workers in the United States. The study included Tepper's (2000) Abusive Supervision Scale and Williams and Anderson's (1991) Interpersonal Citizenship and Interpersonal Deviance Scale. The study found that SDO links positively to interpersonal deviance and negatively to interpersonal citizenship. The study suggests that SDO values may be important for understanding employee inclinations to engage in deviant, counterproductive, or unethical workplace behaviors and to avoid participation in helping or citizenship behavior.

To explore the conditions under which sustained abusive supervisory behavior occurs, Hu and Liu (2017) developed a model using the supervisor's social dominance orientation as a predictor of abusive behavior. The authors pointed out while much research focuses on the consequences of abusive supervision, relatively little research analyzes the antecedents of such behavior. The exception to this are the studies that explore the influence of the abuses experienced by the supervisors themselves (Bushman, Bonacci, Pedersen, Vasquez, \& Miller, 2005), which suggest that abusive supervisors are emulating the behavior of their own leaders (Liu, Kwong Kwan, Wu, \& Wu, 2012), especially under stressful work conditions (Mawritz, Dust, \& Resick, 2014). Hu and Liu (2017) posit that to explain abusive behavior, some motivational rewards must exist and 
that exploring these motivations will contribute to our understanding of the fundamental causes of abusive supervision. Drawing from the literature on strategic bullying (Ferris, Zinko, Brouer, Buckley, \& Harvey, 2007; Shao et al., 2011), which suggests that bullying is motivated by status attainment, the authors theorized a model proposing that individuals high in SDO are more motivated to gain status to facilitate the attainment of dominance over others. In its entirety, the model posits the motivation to enhance one's status is a mediating mechanism between SDO and abusive behavior. Hu \& Liu (2017) suggest other variables (moderators) that may influence the strength of the mediated relationship, including positional instability (a highly competitive environment with continuous performance evaluations), the perception of low internal respect (perception of status in eyes of others), mechanistic organizational structure (centralized authority and close supervision), or a hostile work environment (prevalent aggression or incivility). Thus, supervisors with a high SDO will be more likely to seek status attainment through abusive behavior. This tendency is augmented by the moderating influence of high positional instability, low internal perceptions of respect from subordinates, and a highly mechanistic and hostile work environment.

Aiello et al. (2018) compared employees $(N=538)$ who score high on the SDO measure in the context of hierarchy-enhancing and hierarchy-attenuating organizations to determine which employees endorsed harsh or soft power tactics. Harsh or soft power tactics refer to the amount of leeway given to a subordinate employee in choosing a course of action in response to a request from an influencing agent. The study found that SDO related positively to harsh power tactics and negatively to soft power tactics. Differences existed among hierarchy-attenuating and hierarchy-enhancing work 
environments, with high SDO supervisors in hierarchy-attenuating organizations expressing the strongest opposition to soft power tactics. This finding supported the premise of social dominance theory that individuals high in SDO want to maintain dominant-submissive intergroup relationships.

Individual Characteristics. Feather and McKee (2012) utilized a sample of 170 Australian undergraduate students and employed a path analysis to develop a model exploring the relationship between values, SDO, Right-Wing Authoritarianism, and sexism. The hypotheses guiding the study posited that the respondents' value priorities would affect their response to each item on the scales utilized in the study. The authors argued that a people's values sensitize them to information that is relevant to the fulfilment or obtrusion of those values, and influence the way people respond to information. For example, the assertion in the SDO scale that "Some people are just inferior to others" would be evaluated positively by a person who scores highly on the power value dimension of the Schwartz Value Survey (SVS; Schwartz, 1992). The power value is defined by ratings of the importance of social status and prestige, and control and dominance over people and resources (Schwartz, 2012). The findings provided evidence that power values predict hostile and benevolent sexism towards women via right-wing authoritarianism and SDO. Right-wing authoritarianism (RWA) is a construct that identifies individuals who prefer traditional values, are submissive to authority figures, are significantly ethnocentric, and are predisposed to behave aggressively toward groups perceived as inferior (Altemeyer, 1998). Hostile sexism is grounded in the belief that men are more competent than women and consists of blatant forms of sexism such as endorsement of traditional gender roles (e.g., Feather, 2004; Swim, Mallett, Russo- 
Devosa, \& Stangor, 2005). Benevolent sexism is one component of ambivalent sexism that endorses complementary gender differentiation, heterosexual intimacy and paternalism (Glick \& Fiske, 1996). The path analysis in Feather and McKee's (2012) study is consistent with the assumption that belief-attitude systems, as at those embodied in SDO, express a person's value priorities and that these belief-attitude systems mediate the effects of personal values on prejudice.

Navarrete, McDonald, Molina, and Sidanius (2010) suggest that intergroup bias is fundamentally a gendered phenomenon. According to social dominance theory (Sidanius \& Pratto, 1999), gender differences in SDO exist and are invariant across social, cultural and other contexts, a principle known as the invariance hypothesis (Pratto et al., 1994). All else being equal, men score higher in SDO than women (Pratto et al., 2006; Sidanius $\&$ Pratto, 2012). However, there are inconsistencies in the relationship between gender and SDO in the literature. The inconsistent findings imply that the gender difference in SDO can be explained without recourse to biology (Sidanius, Pratto, \& Mitchell, 1994). Evidence supports the hypothesis that both gender identity (Dambrun, Duarte, \& Guimond, 2004) and masculinity and femininity (Foels \& Pappas 2004; Schmitt \& Wirth, 2009; Snellman, Ekehammer, \& Akrami, 2009) mediate the link between gender and SDO. Gender difference is attenuated or disappears when these factors are statistically controlled. In addition, some evidence shows that characteristics associated with masculinity and femininity (e.g., power, benevolence) also mediate the link between gender and SDO (Caricati 2007). Finally, convincing evidence points to a relationship between social context (e.g., culture, ideology, status) and SDO (Batalha, Reynolds, \& Newbigin, 2011; Zakrisson 2008). 
There are conflicting findings regarding the gender invariance hypothesis. In a seminal study, Pratto et al. (1994) surveyed 1,952 Stanford students to assess their level of SDO and compare that to other demographic and ideological characteristics. They found that men tended to have higher SDO than women and that SDO scores could predict social and political attitudes. The researchers found that higher SDO scores correlated with pursuit of hierarchy-enhancing jobs, ideologies involving group prejudices, support for chauvinistic policies, and political-economic conservatism. Foels and Reid (2010) questioned the invariance hypothesis by exploring the role that social cognitive complexity might have in the gender assumptions of social dominance theory. They cite literature which contextualizes women within low-status groups crossculturally, and which associates social status with cognitive processes. According to this literature, low-status group members are more cognitively complex than high status group members (Lorenzi-Cioldi, 1998). Foels and Reid (2010) adopt Bieri's (1955) definition of cognitive complexity as a multidimensional mental representation of the social world that differentiates between how low-status individuals engage in more cognitively complex views of other individuals compared to high-status individuals. This logic reflects the SDT proposition that high-status groups have higher SDO than lowstatus groups and that members with high SDO view the world as a zero-sum competition, wherein groups compete for a finite amount of social and natural resources (Sidanius \& Pratto, 1999). Because women have lower social status than men, women may be lower in SDO not because of gender but, rather, because their social status facilitates higher levels of cognitive complexity. The authors tested this hypothesis by analyzing data from 117 college students from a large public university. Their findings 
support social dominance theory but raise questions about whether gender or low social status cause the low SDO scores for women. That is, the research supports the core of social dominance theory, but hints at a new path towards low SDO scores: high social status predicts low cognitive complexity, and low cognitive complexity predicts support of group-based hierarchies. The results suggest the possibility that members of socially subordinate, lower status groups (e.g., gender, racial, religious, or sexual minorities) might also be high in cognitive complexity.

In a study examining whether, all else being equal, men really are higher in SDO than women, Batalha et al. (2011) conducted three empirical studies in three different settings comparing men and women under similar cultural contexts. The sample for Study 1 consisted of 423 student participants from Australia and Sweden (172 men and 251 women). Using a hybrid of online and classroom-based questionnaires, the students filled out the original 16-item SDO scale. The result of the factorial ANOVA with SDO as the dependent variable showed that the effect of gender was small and differed significantly between the Australian and Swedish participants. Cultural differences, including a hierarchy-attenuating ideology of gender equality in Sweden, explained the differing SDO scores. Study 2 tested directly the ideological influences of culture on SDO using 108 Swedish university students (54 men and 54 women). Based on previous investigations, the researchers categorized students into hierarchy-attenuating disciplines (HA) or hierarchy-enhancing (HE) disciplines. Hierarchical multiple regression analyses showed that whereas ideological orientation (HE/HA) predicted SDO, gender did not. Study 3 was designed as an experiment ( $N=245$ Australian students, 177 women and 68 men) which shifted status positions among participants by manipulating cognitive tasks 
so that men and women would perform differently. High status was associated with high performance in cognitive tasks that men have been traditionally performed more successfully. The researchers hypothesized that the high-status group would have higher SDO and that men would have higher SDO. They conducted a factor analysis of covariance with SDO as the dependent variable. Low-status participants scored lower in SDO, but while men scored higher in SDO than women in the low-status group, no gender difference was observed in the high-status group.

Zakrisson (2008) attempted to problematize the invariance hypothesis by designing a study to explore gender differences in SDO within egalitarian social structures, such as voluntary organizations and politically egalitarian communities. The resulting study of 831 randomly selected Swedish adults (one of the few randomized samples) from eight municipalities across the country (scored high or low in political equality) who completed the SDO scale revealed that men and women displayed equally low levels of SDO when they occupied similar positions in the status hierarchy of a community.

Schmitt and Wirth (2009) explored the presumed correlation between gender and SDO by trying to contextualize the correlation not in relation to the social environment, but to the social-psychological causes which might give rise to the gender difference. They contribute to the explanatory literature by examining two processes that might explain the gender differences in SDO: self-stereotyping into traditional gender roles and group-interested responses that support patriarchy. They utilize social identity theory to establish the foundation for group responses associated with SDO. Because group identity is strongly associated with the desire to protect the in-group's position in respect 
to other groups, a high SDO by men could reflect their desire to maintain patriarchal hierarchy in society. Similarly, in-group members tend to see themselves and other ingroup members as representative of the in-group and its associated stereotypes. Men and women could respond to SDO questions because they have internalized the gender stereotypes as part of their gender group identities. The study suggests that gender differences in SDO may stem from gendered self-stereotyping.

The authors hypothesize that two specific elements of gender group identity could account for the higher SDO among men relative to women. The first is a response to patriarchy that reflects the interest of the gender in-group, as measured by hostile and benevolent sexism. The second dynamic would be the process of stereotyping in gender terms. The sample of 72 psychology undergraduate students completed, as part of their class assignment, three instruments: the Ambivalent Sexism Inventory (ASI) to measure sexism, the Bem Sex Role Inventory to measure masculine and feminine dimensions, and the SDO scale. Using a multiple regression model predicting SDO, the authors tried to ascertain whether gender self-stereotyping and group interests were independent mediators of the gender differences in SDO attitudes. The results confirmed that gender differences in femininity fully mediated the gender differences in SDO.

Emotions. Few studies have examined the relationship between SDO and emotions. The developers of SDT did not discuss its emotional correlates. One study investigating the relationship between SDO and the expression of emotions indicated that social dominators tend to withhold emotional expressions (Van Hiel et al., 2006). The authors suggest that theoretical models that incorporate emotions and SDO to predict prejudice should be explicit about the aspect of emotion that they are studying. This study 
measured three aspects of emotion. The first measure was emotions in the context between positive and negative emotions (measured by the Positive and Negative Affect Schedule; PANAS; Watson, Clark, \& Tellegen, 1988). The second measure was affect intensity (measured by the Emotional Intensity Scale, EIS; Bachorowski \& Braaten, 1994; Geuens \& De Pelsmacker, 2002). The third measure was affect expression (Emotional Expressiveness Questionnaire, EEQ; King \& Emmons, 1990). The study concluded that emotion expression yielded the strongest main and moderator effects in the regression analysis, indicating that emotion expression is an important but understudied variable in predicting racism.

In a novel study of emotions designed to predict prejudice, Hodson and Costello (2007) found that interpersonal-disgust sensitivity was a strong predictor of negative attitudes towards immigrants, foreigners and other stigmatized groups. The study conceptualizes disgust with the need to avoid offensive stimuli instead of initiating a fight response. A group of 103 English Canadian psychology students completed a battery of questionnaires measuring the links between disgust and prejudice. They indicated their attitudes towards various groups in a widely used and well validated "feelings thermometer," and they completed a 7-item Modern Racism Scale, which the authors modified to gather information about immigrant groups. In addition, the participants completed the 32-item Disgust Scale to assess their reactions to specific disgust scenarios and stimuli. The authors felt that it was necessary to control for the very real fear of contracting a contagious disease from the "other" and wanted to differentiate between responses motivated by the fear of disease from pure disgust, so they used the 18 -item Perceived Vulnerability to Disease Scale to tap this dimension of interaction. In addition, 
the subjects completed three other instruments: the Ten-Item Personality Inventory (TIPI; Gosling, Rentfrow, \& Swann, 2003) to provide information on how the Big-Five personality factors influence attitudes towards groups), the original 16-item SDO Scale and a shortened version of the RWA scale to assess ideological orientation. A series of regression analyses uncovered a strong relationship between higher interpersonal disgust sensitivity and high SDO and RWA as well as with a variety of other negative reactions against immigrant and foreigners. The path analysis derived much of its explanatory power by placing SDO (and RWA) and moderators of attitudes towards immigrants. The authors highlight how knowledge of how emotions relate to ideological beliefs is a crucial step in understanding prejudiced individuals.

The emotion of disgust worked its way into an analysis conducted by Matthews and Levin (2012). The authors designed two studies to explore the relationship between perceptions of threat posed by Muslims and the intergroup emotions directed towards this group. In the first study $(N=109)$ students completed shortened versions of scales (30 minutes of total study time), which measured their personality characteristics as well as their ideological (SDO and RWA) orientations. Path analyses revealed that participants scoring highly on the SDO items felt threatened economically by Muslim immigrants. This perception of economic threat, in turn, predicted the intergroup emotions of anger and disgust. In the second study, randomly assigned undergraduate students $(N=162)$ read and responded to an article that portrayed Muslims as either opposing or appreciating the norms and values of the Western world. After reading the article, the participants responded to questions designed to gauge their perceived threat from Muslims as well as a set of items regarding their emotions and behavioral inclinations 
towards Muslims. A path analysis (mediational analysis) found that disgust was a stronger emotion resulting from perceived threat to the values of Western society.

In a study conducted with Polish students $(N=175)$ and adults $(N=197)$, Hiel and Kossowska (2006) explored the relationship among the variables of what they call "submissive authoritarianism," measured by the RWA Scale, and "dominant authoritarianism," measured by the SDO Scale, and measures of positive and negative affect. Specifically, they attempted to discern the relationship between SDO, RWA, and negative and positive emotions, and test moderator models in which the emotion variables moderated the RWA and SDO effects on racism. Besides the SDO and RWA scales, the researchers used the Positive and Negative Affect Schedule (PANAS), the Emotional Intensity Scale (EIS), and the Emotional Expressiveness Questionnaire (EEQ). Racism measures were translated from the work of Kleinpenning and Hangendoorn (1993) because of the items' ability to measure various roots of prejudice and reactions to racism, from the biological (based on biological reasons for racist views) to the symbolic (based on cultural dimensions of racism). In general, the results showed a strong and consistent relationship between the items of the EEQ and the SDO measures. High scores in the SDO measures, accompanied by low emotional expression measures, led to the highest level of prejudice indicating that social dominators tend to keep their emotions to themselves.

\section{Intergroup Dominance and Intergroup Antiegalitarianism}

Recent evidence suggests that the construct of SDO breaks down into two specific sub-dimensions that include support for intergroup dominance (SDO-D) and intergroup antiegalitarianism (SDO-E; Ho et al, 2012; Ho et al., 2015). Ho and colleagues (2012) 
were the first scholars to empirically test the possibility that SDO has two distinct dimensions. They found that the SDO scale consists of two related dimensions, SDO-D and SDO-E, and that the two dimensions predicted different outcome variables concerning group-based dominance and antiegalitarianism. Intergroup dominance (SDOD) was defined to reflect the original definition of SDO developed by Sidanius and Pratto (1993). Intergroup dominance (SDO-D) is characterized as a disposition to support group-based dominance with an active oppression of subordinate groups. On the other hand, SDO-E concerns a disposition to oppose group-based equality through policies that limit access to resources for members of subordinate groups (Ho et al., 2012). Having two dimensions of SDO allows the exploration of specific facets of prejudice and discrimination, and other intergroup relations (Ho et al., 2012).

With the development of the new $\mathrm{SDO}_{6}$ scale, Ho et al. (2012) identified that SDO-D was related to forceful subjugation of outgroups, endorsement of immigrant, persecution, old-fashioned racism, zero-sum competition, and support for war. Intergroup antiegalitarianism (SDO-E) was linked to noninclusive and nonegalitarian preferences regarding intergroup relations. Ho and colleagues (2012) suggest that different types of prejudice are related to the two SDO dimensions. The study distinguishes between oldfashioned racism (belief in outgroup inferiority and legitimizing dominance; SDO-D) and modern forms of prejudice (support for group inequality; SDO-E). Intergroup dominance (SDO-D) best predicted aggressive behaviors and old-fashioned racism, while SDO-E best predicted subtle hierarchy-enhancing ideologies and modern forms of prejudice.

In 2015, Ho and colleagues developed the most current $\mathrm{SDO}_{7}$ scale for measuring the theoretically grounded dimensions of SDO-D and SDO-E. Data were collected from 
seven samples from the United States that were comprised of 2,655 participants. The new $\mathrm{SDO}_{7}$ scale's predictive validity was consistent with the $\mathrm{SDO}_{6}$ 's (Ho et al., 2012), with SDO-D predicting overt forms of racism (intergroup dominance), and support for aggression and violence toward lower status groups. While SDO-E was a more important predictor of political conservatism, unequal distribution of resources, opposition to policies that address equality, and covert forms of prejudice. The $\mathrm{SDO}_{7}$ revealed personality and individual differences between SDO-D and SDO-E. The SDO-D dimension was more related to Machiavellianism, narcissism, and psychopathy than the SDO-E dimension.

Kiteily et al. (2015) conducted a study of blatant and subtle dehumanization that included using the SDO-D and SDO-E dimensions of social dominance. The authors defined blatant dehumanization as a psychological process that strips others of group identity and excludes them from moral consideration. Seven studies in three countries found that blatant dehumanization was strongly associated with SDO-D and unrelated to SDO-E. The study supported the hypothesis that individuals who believe that certain groups are superior to other groups are more likely to engage in blatant dehumanization. This finding highlights the aggressive nature of SDO-D.

Hindriks et al. (2014) reported similar findings validating the distinction between SDO-D and SDO-E using a representative sample $(N=802)$ of Dutch natives. The authors suggest that in this population, individuals with high SDO-D feel free to overtly express prejudice and discrimination. On the other hand, individuals high in SDO-E tend to prefer covert and subtle expressions to justify the status quo. 
Bergh et al. (2015) explored the personality and socio-political correlated of both SDO-D and SDO-E in a study conducted with a sample of $(N=5,741)$ of European and Maori participants in New Zealand. Bergh and colleagues found that the personality roots of SDO-D and SDO-E differ between the two subdimensions. A strong negative relation was found between the personality trait of honesty-humility and SDO-D, while it was unrelated to SDO-E. Intergroup dominance (SDO-D) had a positive relationship with hostile and benevolent sexism, while SDO-E was primarily associated with political conservatism.

\section{Emotion Regulation}

The concept of "emotion" refers to an array of responses and this impedes efforts to operationalize a definition of the term (Gross, 2014). The modal model of emotion posits that emotions involve person-situation transactions that require attention, have significance to an individual, and give rise to responses that may modify the personsituation relationship in critical ways (Barrett et al., 2007; Gross,1998a ; Gross \& Thompson, 2007). Meta-analyses have shown how emotions consistently affect cognitive and behavioral outcomes (Angie et al., 2011; Hülsheger \& Schewe, 2011; KammeyerMueller et al., 2013; Lench et al., 2011; Mesmer-Magnus et al., 2012). Emotions can influence decision-making and affect interpersonal interactions (Fiske, 1998; Gross, 2014; Gross \& Thompson, 2007). What we experience and categorize as emotion powerfully affects the way we perceive and engage with the world (Stefanucci et al., 2011) and our emotional processes carry implicit information about the goodness and badness of the people and things around us (Clore \& Tamir, 2002). Emotions have a wide, sometimes unwelcome, influence on many aspects of the work environment 
(Barsade \& Gibson, 2012), and at times need to be regulated by the individual experiencing them (Gross, 2013, 2014; Hochschild, 1983).

Emotion regulation is a broad set of activities that may be considered emotional labor when certain criteria are met (Grandey, 2013; Grandey \& Melloy, 2017). Emotional regulation performed in the workplace to meet the display rules established by the employer is called emotional labor, a term coined by Hochschild in 1983 from her seminal work with flight attendants. Emotional labor (Hothschild, 1983) is a distinct from emotion regulation (Gross, 1998b) in that emotional labor requires workers to manage feelings and expressions to help the organization profit (Grandey, 2013).

Grandey (2000) identified the need to go beyond the notion of emotional labor developed by Hochschild (1983). Grandey suggests that specific strategies of emotion regulation are necessary to understand how employees manage their emotions at work. Based on the earlier work of Grandey (2000), Grandey and Gabriel (2015) proposed linking the emotional labor and emotional regulation literatures. Emotion regulation seeks to understand the processes that individuals use to manage their emotions (Gross, 1998). Emotional labor, on the other hand, deals with emotions as a means of meeting organizational display rules (Hochschild, 1983). The merging of the two organizational emotion research traditions opens an opportunity for researchers to better understand the role employers and their employees have on important employee and organizational outcomes such as job satisfaction, burnout, and interpersonal performance. The inclusion of organizational display rules, employee emotion regulation strategies, and emotion performance offers organizational researchers an opportunity to further explore emotions at work (Grandey, 2000; Grandey \& Gabriel, 2015). 
Hochschild (1983) conducted an extensive analysis of the structure of emotions in work environments. She argues that the work environment requires that employees express only emotions appropriate to their occupations and work settings. Hochschild (1983) uses the term "feeling rules" to describe organizational norms about the appropriate type and amount of feeling that should be experienced in a particular work situation. Emotion regulation (Gross, 1998a) refers to the actual strategies employees use to perform the emotion work required to accommodate the feeling rules established by the organization. The flight attendants in Hochschild's study, for example, understand that the emotional expectations of their job require them to suppress, rather than express, their emotions when confronted with unpleasant circumstances.

Research on emotion regulation focuses on employee-customer interactions, leaving emotion regulation in employee-employee interactions relatively unexplored (Grandey \& Gabriel, 2015; Kim et al., 2013). Employees may also regulate emotions to engage in impression management or to establish social relationships when dealing with a supervisor or coworkers (Pearson et al., 2000). All jobs entail some degree of emotion regulation as employees, explicitly or implicitly, follow the emotional display rules for the appropriate expression of emotions on the job (Diefendorff et al., 2005); not only in interactions with customers, but also in interactions with colleagues, supervisors, or even subordinates (Grandey, 2000; Grandey \& Gabriel, 2015). For example, demographic diversity may be a source of emotion regulation in work environments because organizations generally require employees to hide personal biases (Kim et al., 2013). Kim et al. (2013) theorize that working in a demographically diverse workgroup (in terms of age, race, and gender) increases response-focused emotion regulation (i.e., surface acting, 
which involves modifying external expressions) because of demography-related in-group favoritism and out-group discrimination. Social psychologists have long recognized that prejudices are influenced by perceivers' emotional states (Dasgupta, DeStenio, Williams, $\&$ Husinger, 2009). Employees can and do regulate their emotions to meet societal, occupational, and organizational norms, and the two processes form an emotion regulation framework that includes organizational display rules, emotion regulation (cognitive reappraisal or expressive suppression), and behavioral manifestations of emotions that affect individual and organizational outcomes (Grandy, 2000; Hochschild, 1983). In the following section the researcher discusses the literature pertaining to organizational display rules, emotion regulation strategies, and affective and social outcomes of emotion regulation.

\section{Organizational Display Rules}

Different industries develop different emotional cultures that dictate which emotions need to be expressed, and which need to be inhibited. Employees must therefore manage their emotions to fit their organization's emotional culture and climate. When asked about their emotion regulation, people often describe efforts to downregulate negative emotions, with a particular focus on decreasing the experiential and behavioral aspects of anger, sadness, and anxiety (Gross, Richards, \& John, 2006). For example, flight attendants limit hostile feelings toward unpleasant passengers (Hochschild, 1983).

Interactions between employees and customers are important sources of emotion management because of the influence of employees' emotions on clients' purchase behaviors (Rind \& Strohmetz, 1999), the need to maintain the organization's image 
(Gutek, Cherry, \& Groth, 2002), and the stress associated with interactions such as incivility or aggression (e.g., Grandey et al., 2004; Lee \& Brotheridge, 2006). In demographically diverse workplaces, members of dominant groups (e.g., White people) often regulate emotions to avoid seeming prejudiced (Grandey \& Gabriel, 2015; Kim et al., 2013).

\section{Emotion Regulation Strategies}

Emotions involve changes in the subjective experience and the expressed behavior that follows the experience (Mauss, Levenson, McCarter,Wilhelm, \& Gross 2005). The modal model of emotion unites features common to many different approaches to emotion (Barrett et al., 2007; Gross, 1998b). The modal model of emotion begins with a psychologically relevant situation. This situation can be defined by features of the external environment or by the activation of internal responses. Emotions in the workplace are helpful when they appropriately guide and motivate socially appropriate behaviors (Averill, 1980). Examples of helpful emotions include episodes of fear that lead us to avoid potentially deadly fights, episodes of happiness that reinforce new friendships, and episodes of anger that propel us to fight for causes we care about. Emotions are harmful when they are the wrong intensity, duration, frequency, or type for a particular situation (Gross, 2014). Examples of unhelpful emotions abound, including anger that leads one to harm oneself or a loved one, laughter that gives offense, or anxiety that cripples one socially.

Although five sets of emotion regulation strategies can be identified along the emotion process (see Gross, 2001), cognitive reappraisal and expressive suppression have been identified as the ones that people use more frequently in everyday life (Gross, 2014; 
Gross \& John, 2003; Hochschild, 1983). Researchers have distinguished between antecedent-focused emotion regulation (Lazarus \& Alfert, 1964) and response-focused emotion regulation strategies (Gross, 1998b; Hochschild, 1983). Antecedent-focused (cognitive reappraisal) strategies occur before the emotion response tendencies have activated and have changed behavior and physiological responding (Gross, 1998b; Hochschild, 1983). Response-focused (expressive suppression) strategies occur when an emotion is already underway, after the generation of response tendencies (Gross, 1998b; Hochschild, 1983).

Cognitive reappraisal. The emotion regulation strategy of cognitive reappraisal involves construing a potentially emotion-eliciting situation in a way that changes its emotional impact (Gross \& John, 2003; Hochschild, 1983; Webb et al., 2012). Cognitive reappraisal influences whether particular emotion response tendencies are triggered and are therefore expected to have generally positive implications for affective and social functioning (Gross \& Thompson, 2007; Grandey, 2000; Gross, 1998b; Hochschild, 1983; Liu et al., 2010; Webb et al., 2012). Reappraisal allows for changes in both the emotional experience and the emotional expression of that experience (Gross, 2002; Koole, 2009). In the emotional labor literature, individuals use cognitive reappraisal in the attempt to modify feelings to match the required display rules of the organization (Gabriel, Daniels, Diefendorff, \& Greguras, 2015). In other words, individuals try to align required and true feelings when they engage in this form of emotion regulation.

Expressive suppression. In contrast to cognitive reappraisal, expressive suppression of emotion influences how emotion response tendencies are modulated once they have been triggered and are therefore expected to have generally more negative 
implications for affective and social functioning (Gross, 1998b; Gross \& Levenson, 1993; Hochschild, 1983). In other words, individuals engaging in emotion suppression are putting on a mask by suppressing, amplifying, or faking emotions (Hochschild, 1983; Gabriel et al., 2015). This practice entails experiencing emotional dissonance because feelings and actions diverge (Hochschild, 1983). Studies show that employees who report regularly having to display emotions that conflict with their own feelings are more likely than others to experience emotional exhaustion (Van Dijk \& Brown, 2006; Glomb \& Tews, 2004; Zapf \& Holz, 2006). Furthermore, emotion suppression is linked to impaired memory, greater experience of negative emotions, lessened social support, increased avoidant behaviors, and more depressive symptoms in comparison with the emotion regulation strategy of cognitive reappraisal (Gross, 2002; Gross \& John, 2003; John \& Gross, 2004). Finally, emotion suppression affects only emotional expression and does not change the emotional experience (Gross, 2002; Koole, 2009).

\section{Affective and Social Outcomes}

Considerable research indicates that the regulation of one's emotions contributes to various important outcomes. Emotion regulatory processes are central to mental health; they can either support or disrupt the capacity to work, relate to others, and feel enjoyment (Gross \& Munoz, 1995). Greater use of cognitive reappraisal was associated with positive outcomes such as greater psychological adjustment and improved interpersonal functioning, whereas greater use of suppression was negatively related to such outcomes (Gross, 2002; John \& Gross, 2004; 2007). Suppressing negative emotions left the experience of negative emotions intact, while suppressing positive emotions decreased the experience of positive emotions (Gross \& Levenson, 1997; Nezlek \& 
Kuppens, 2008). Successful emotion regulation allows people to achieve a wide range of beneficial outcomes, including better mental health (Aldao, Nolen-Hoeksema, \& Schweizer, 2010), physical health (Williams et al., 2015), interpersonal relationships (Häfner \& IJzerman, 2011), and work performance (Jiang, Zhang, \& Tjosvold, 2013). It is therefore important to understand how people regulate their emotions and the factors that influence their success in so doing.

A mismatch between how one feels and how one behaves depletes cognitive and physiological resources (Grandey, 2000, 2003; Gross \& John, 2003), impairing memory for the emotion-triggering event and potentially intensifying the original emotion experienced (Lerner, Li, Valdesolo, \& Kassam, 2015). Expressive suppression has a negative impact on social interactions compared to cognitive reappraisal (Butler et al., 2003; Gross, 2002). Previous studies have examined how individuals' attempts to reduce their negative emotions are related to well-being outcomes (Gross, \& Muñoz, 1995; Kashdan, Barrios, Forsyth, \& Steger, 2006), dispositional variables (Lischetzke \& Eid, 2006), and general behavioral disorders (Aldao et al, 2010). The expressive suppression of negative emotions is a commonly used emotion regulation strategy in social life (Gross, Richards, \& John, 2006). Suppressing negative emotions in social situations is related to negative social consequences (e.g., Butler et al., 2003; Butler, Gross, \& Barnard, 2014).

Individual differences in adaptive forms of emotion regulation (such as cognitive reappraisal) have cumulative benefits for affective functioning, social interactions, and well-being, whereas individual differences in maladaptive forms of emotion regulation (such as expressive suppression) have cumulative costs for affective, social, and well- 
being domains (Gross \& John, 2003; Nezlek \& Kuppens, 2008). Higher levels of cognitive reappraisal use protect against cardiovascular disease, whereas higher levels of suppression may place a person at higher risk for various cardiovascular diseases (Appleton \& Kubzansky, 2014; Gianaros et al., 2014; Trudel-Fitzgerald, Qureshi, Appleton, \& Kubzansky, 2017).

Emotion regulation is almost always a social affair as emotion regulation occurs primarily in interpersonal contexts (Gross et al., 2006). Butler et al.'s (2003) pioneering research provided initial causal evidence for the social consequences of emotion suppression by experimentally manipulating emotion regulation. Expressive suppression has negative consequences including poor interpersonal coordination, decreased feelings of rapport and affiliation, and increased negative feelings about the interaction (Butler et al., 2003).

Individuals engage in emotion regulation primarily to reduce the experience of negative emotions (Gross et al., 2006). Employees who foster negative attitudes toward coworkers may experience negative emotions such as hostility or emotional conflict (Kim et al., 2012; Jehn, Northcraft, \& Neale, 1999). Research on emotion regulation focuses on customer interactions, leaving emotion regulation in employee-employee interactions relatively unexplored (Grandey \& Gabriel, 2015). Kim et al. (2013) theorize that working in a demographically diverse workgroup (in terms of age, race, and gender) increases response-focused emotion regulation strategies, such as expressive suppression because of demography-related in-group favoritism and out-group discrimination. Employees who foster negative attitudes toward dissimilar coworkers may experience negative emotions, such as hostility or emotional conflict (Kim et al., 2013; Jehn et al., 1999). 
Demographic diversity may be a source of emotion regulation in work environments because organizations generally require employees to hide their personal biases (Kim et al., 2013). Organizational emotional display rules have been positively related to expressive suppression of emotions (Allen, Pugh, Grandey, \& Groth, 2010; Grandey \& Melloy, 2017; Lee \& Ok, 2012).

\section{Instigated Incivility}

The concept of workplace incivility was introduced by Andersson and Pearson (1999) to the management literature to explore how increasingly aggressive behaviors start with less intense behaviors such as incivility. Andersson and Pearson defined workplace incivility as a "low-intensity deviant behavior with ambiguous intent to harm the target, in violation of workplace norms for mutual respect. Uncivil behaviors are characteristically rude and discourteous, displaying lack of regard for others” (p. 457).

Two recent reviews of the literature on workplace incivility highlight the significant disproportion in studies that focus on victims of workplace incivility versus studies that focus on the instigators of workplace incivility (Cortina et al., 2017; Schilpzand et al., 2016). The following section presents a review of the instigated incivility literature. This literature review on instigated incivility is organized in two sections, (a) individual factors, and (b) organizational factors that are known to predict, mediate, or moderate instigated workplace incivility.

\section{Individual Factors}

Cortina et al. (2001) conducted a foundational and focused treatment of the topic of incivility that showed that high levels of power, and gender positively relate to incivility. At that time, the study was innovative in its expansion of the literature on 
workplace mistreatment beyond its more overt manifestations (violence, aggression, bullying) by differentiating between the incidence, the targets, the instigators, and the outcomes of incivility. A sample of $(N=1,180)$ public sector employees of a Federal Court system completed a pencil-and-paper mail survey. The survey battery included the Workplace Incivility Scale (WIS); an abbreviated version of the Job Descriptive Index (JDI) which measures various dimensions of job satisfaction; an abbreviated version of the Mental Health Index (MHI) to measure psychological and health-related outcomes of incivility; and a version of the Perception of Fair Interpersonal Treatment Scale (PFIT) to assess the nature of personal workplace relationships. After validating the WIS by means of a confirmatory factor analysis, the multivariate analysis yielded foundational results in incivility research. Not only did the research reveal the ubiquitous nature of incivility (over two thirds of respondents reported being victims) but it discovered that incivility is a gendered phenomenon with respect to its target. The study also showed those in certain job positions experienced lower rates of incivility than others did.

Daniels et al. (2015) were the first scholars to explore the relationship between social dominance orientation (SDO) and instigated incivility. The study tested a model of incivility that proposes a relationship between SDO and instigated incivility mediated by psychological entitlement. Psychological entitlement was defined as a stable and pervasive belief of being entitled, and characterized by lack of concern for the feelings of others. Data were collected from two convenience samples $(N=321 ; N=142)$ to avoid the concerns pertaining to single-study designs. The results from both samples indicated a relationship between SDO and instigated incivility that was mediated by psychological 
entitlement. This study was published in the Academy of Management Annual Meeting Proceedings, so details of the study are very limited.

Other scholars found a pattern within instigated incivility that indicates that men are more likely than women to instigate incivility (Crampton \& Hodge, 2008; Gallus, Bunk, Matthews, Barnes-Farrell, \& Magley, 2014; Pearson et al., 2000; Reio \& Ghosh, 2009). Research conducted by Crampton and Hodge (2008) found that males committed $70 \%$ of the cases of reported instigated incivility and that women were more likely to report experiencing workplace incivility than men.

Several incivility scholars identified negative affectivity as personality characteristic that may be associated with incivility (Ghosh, Dierkes, \& Falletta, 2011; Jex, et al., 2010; Meier \& Semmer, 2012; Reio \& Ghosh, 2009). Negative affectivity is a dispositional trait related to negative emotionality and self-concept, such that individuals high in negative affectivity tend to focus more often on negatives and suffer from low self-esteem (Siomkos, Rao, \& Narayanan, 2001). Looking at the relationships among negative affectivity, perceptions of organizational justice, and vindictiveness, Skarlicki and Folger (1997) found a positive relationship between negative affectivity and organizational vindictiveness and a negative relationship between vindictiveness and perceptions of organizational justice. These results support the idea that negative affectivity is an antecedent of incivility. Negative affect is mood disposition that includes felt negative emotions (aversive emotions including anger, disgust, contempt, fear, guilt, and nervousness) and negative expressed behavior (Clark, Watson, \& Mineka, 1994; Watson, Clark, \& Tellegen, 1988). Understanding employee affective experiences gives HRD professionals the opportunity to design interventions that help organizations 
promote positive emotional workplace experiences and therby improve employee health and job satisfaction (Reio \& Ghosh, 2009).

Meier and Semmer (2013) indicated that instigator characteristics such as trait anger positively relate to instigated incivility. Trait anger reflects a proneness to anger or the tendency to experience state anger (Douglas \& Martinko, 2001); it is thus considered part of an individual's stable personality. In a study designed to examine antecedents of uncivil behavior towards coworkers and supervisors, the authors investigated the role of work characteristics, personality, and work-related anger simultaneously. The authors hypothesized that anger mediates the relationship between lack of reciprocity and incivility, and that this mediation was particularly strong among narcissistic employees.

The findings of Porath \& Pearson (2012) suggest that anger is associated with a higher degree of aggressive behavior, whereas fear was associated with higher levels of indirect aggression towards incivility instigators (Porath \& Pearson, 2012). This illuminates how stepwise escalation, due to emotional responses, may lead to incivility spirals. Douglas and Martinko (2001) found trait anger and negative affect were significant predictors of workplace aggression. Specifically, individuals with higher levels of trait-anger and negative affect are more likely to exhibit both subtle and overt workplace aggression.

Similarly, neuroticism is associated with incivility. Neuroticism is defined as the tendency to experience negative emotions, such as anger, anxiety, or depression (Costa \& McCrae, 1992). Employees high in neuroticism have lower task performance (Tett, Jackson, \& Rothstein, 1991), and are more likely to be dissatisfied with their work demands, their co-workers, and their salary (Denton, Campbell, \& Johnson, 2009). 
Additionally, higher levels of neuroticism are associated with higher levels of vindictiveness, high need for achievement, low self-esteem, low self-monitoring, high narcissism, hostile attribution style, and high passive aggression (Denton et al., 2009; Vardi \& Weitz, 2004).

Agreeableness is an important characteristic in creating conflict free environments in organizations (Chiaburu, Oh, Berry, Li, \& Gardner, 2011). As noted in Barrick, Steward, and Piotrowski (2002) agreeable individuals are more likely to attempt to create a harmonious interpersonal environment due to their desire to work in a nonconflictive environment and thus behave as good organizational citizens. Agreeable individuals, who are sympathetic, cooperative, and trusting (Costa \& McCrae, 1992), are drawn toward quality social interactions and are better team players (Mount, Barrick, \& Stewart, 1998). This leads them to get along with others (Chiaburu et al., 2011).

Trudel and Reio (2011) recognized that the rapidly changing nature of work is creating heightened stress on management and workers and explored how conflict management techniques can influence the manifestation of incivility and other workplace conflicts. In fact, in reviewing the theory and empirical work linking management styles to incivility, the authors concluded that workplace incivility and conflict management styles are linked constructs. The study surveyed 289 employees of three midwestern companies to assess the relationship between conflict management styles and workplace incivility among instigators and targets. Hierarchical regression analysis suggested that some of the conflict resolution styles identified in the literature (integrating, dominating, accommodating, avoiding, and compromising) are more effective in managing conflict leading to uncivil behavior. Specifically, integrating and dominating management styles 
predicted incivility in both the instigator and the target. A dominating style of conflict management predicted more frequent of incivility, while an integrating style reduced its likelihood. The study has significant implications for HRD professionals who deal with the problem of workplace incivility. The authors suggest assessments and screenings for conflict management style as part of the employee recruitment process. The link between conflict management styles and workplace incivility lets HRD practitioners design and implement training solutions that address appropriate conflict management strategies.

Birkeland and Nerstad (2016) examined the impact of obsessive passion for work on incivility instigation. They conducted a 3-wave longitudinal study among 1,263 employees of a large Norwegian workers' union over a 10-month period. The research presented, in their view, two contributions to the literature. Drawing from selfdetermination theory, they posited that how individuals internalize their work into their identity influences incivility perpetration. Obsessive passion is conceptualized as being motivated by gains not associated with job enjoyment or self-development but by the need to maintain high social status and self-esteem. When status is threatened, incivility is more likely to ensue. They hypothesize that high obsessive passion relates positively to incivility instigation at time 1 (H1) and over time (H2). Secondly, they draw upon the literature detailing the dynamics of person-environment fit, specifically achievement goal theory (AGT), to posit that a poor fit between individual values and the organizational values might increase individuals' tendency to act uncivilly. The motivational culture of an organization establishes employee goals, evaluation procedures and the parameters of interactions among employees on work-related tasks. AGT conceptualizes two types of motivational cultures: mastery climate and performance climate cultures. A "mastery 
climate" culture relies more on values associated with effort, self-improvement, personal progress, skill development and cooperation. The work process, in other words, is viewed as one leading to learning and achieving mastery. In contrast, the "performance climate" culture promotes competition and comparisons among employees. This workplace environment, argue the authors, nurtures more maladaptive behaviors. This leads them to hypothesize that a mastery climate moderates the relationship between obsessive passion and incivility at time $1(\mathrm{H} 3)$ and over time $(\mathrm{H} 4)$. Appropriate scales from the literature measured passion for work, motivational climate, and incivility. The results of the analysis supported $\mathrm{H} 1$, that obsessive passion is positively related to incivility instigation at time 1, but did not support $\mathrm{H} 2$ because there was no increase in instigation over time related to obsessive passion. The third hypothesis, positing that a perceived mastery climate would create a more positive relationship between passion and incivility was upheld. The hypothetical extension of this relationship over time, however, (H4) was not supported.

\section{Organizational Factors}

Andersson and Pearson's (1999) seminal article that introduced the construct of workplace incivility to the management literature included a reference to workplace norms in defining workplace incivility - uncivil behavior is "in violation of workplace norms for mutual respect” (p. 457). Incivility researchers have identified workplace norms as a significant factor in the study of workplace incivility (Cortina et al., 2017; Schilpzand et al., 2016). Walsh et al. (2012) developed and validated a measure of civility norms to assess workgroup climate for civility. The measure was designed to assess employee perceptions of workgroup norms of respectful behaviors in the 
workplace. The study included five samples of $(N=2,711)$ employees from diverse organizations. The researchers tested the hypothesis that workgroup civility climate reduces incivility. The results of the study indicate that a climate of workgroup civility significantly moderates workplace incivility.

Gallus et al., (2014) examined incivility from both the perspective of the target and the perpetrator within a broader organizational context of workplace culture. The researchers used a web-based sampling methodology resulting in valid responses from 234 participants, all of whom worked full time as managers or workers in a variety of industries. They explored the interaction among three predictors of incivility perpetration: experienced incivility, the gender of the target and the perpetrator, and the extent to which the organizational climate restricted or permitted incivility. Three hypotheses were tested: 1) the greater the incivility experience, the greater the incivility perpetration, 2) organizational climate moderated the relationship between incivility experiences and perpetration, (i.e., individuals working in an uncivil work environment are nore likely to perpetrate incivility than those working in a civil work environment), and 3) these relationships differ by gender such that the men are more influenced by context (uncivil work environments will generate more uncivil behavior in men than in women). All the hypotheses were supported with important caveats. Men working in uncivil environments reported higher rates of incivility perpetration even if they had not experienced incivility themselves. On the other hand, women seemed unaffected by organizational climate towards incivility. For women, experiencing incivility increased perpetration in civil as well as in uncivil organizational environments. Another interesting finding was that $70 \%$ of the sample reported being both targets and perpetrators of incivility. 
Interpersonal mistreatment at work often occurs in the presence of others. However, these "others" are rarely examined in empirical research despite their importance to the context of the negative interaction. Reich and Hershcovis (2015) conducted two experiments designed to evaluate how witnessing incivility affects observer reactions towards instigators and targets. The authors hypothesized that observers would engage in positive behavior towards the target. This hypothesis was not supported. The authors concluded that bystanders might not perceive ambiguous mistreatment as important enough to warrant intervention. The study highlights the importance of emotions in observer reactions. Reich and Hershcovis found that even when transgressions are ambiguous and low intensity, the erosion of reputation and social relationships might be a cost paid by instigators. The researchers concluded that observers have an immediate negative reaction toward instigators, but not toward targets. Instigators might have more to lose, in the long run, than targets of incivility.

The relationship between instigated incivility, witnessing incivility, and negative workplace outcomes is the focus of work by Holm and colleagues (2015). A large sample ( $N=2871)$ of Swedish hotel and restaurant workers completed an online questionnaire consisting of the workplace incivility scale, a subscale of the Copenhagen Psychosocial Questionnaire (COPSOQ) to measure organizational factors as well as negative outcomes from incivility encounters. The purpose of the study was to gauge whether experience as well as observed incivility resulted in negative outcomes for the target/witness (wellbeing, job satisfaction, turnover intentions, sleeping problems). The COPSOQ also includes measures of organization factors, social support, perceptions of control and job demand. The hypothesis concerned whether experienced and witnessed workplace 
incivility was related to instigated incivility. The structural equation modeling analysis suggested that witnessing coworker incivility explains instigated incivility. There was a strong relationship between witnessing coworkers' incivility and instigating incivility. To some extent, being a target of a supervisors' incivility was related to instigating incivility. Being targeted by a coworker directly related to the negative outcomes measured, but in an interesting addition to the literature, negative outcomes were related to witnessing supervisor incivility.

Van Jaarsveld, Walker and Skarlicki (2010), explored the intricate relations between customers and employees in the service sector, and their association with incivility. Building on research showing that acts of incivility initiated by employees often lead to the target reciprocating with uncivil behavior, the researchers tried to evaluate whether there was a spiral of incivility between employees and customers. After a thorough review of the literature on the mediating role played by the stressors of job demand and emotional exhaustion on incivility, the authors theorized that customers' uncivil behavior toward employees is linked to employee incivility towards customers via job demands and emotional exhaustion. The authors found that uncivil treatment by customers was associated with the higher job demand and emotional exhaustion of employees, which, in turn, related to higher levels of employee incivility towards customers.

Meier and Gross (2015) also address subordinate/supervisor conflict. Using a novel research design, an interaction-record diary, the authors examined the effect of supervisors' incivility toward workers on instigated incivility against the supervisor by the same workers. The data reported are derived from brief surveys completed by the 
participants $(N=131)$ immediately after interacting with a supervisor. The participants reported on their supervisor's behavior (experienced incivility) and their own behavior (instigated incivility) with a single item each, scaled from "very civil" (1) to "very uncivil" (7). In addition, each participant completed a morning questionnaire (a shorted version of the Profile of Mood States) to assess the exhaustion level of the worker heading into the day. Experienced incivility did result in reciprocal response from the target, initiating a "vicious cycle," but the duration of the effect was short. Indeed, the role of time in incivility instigation is one of the major contributions of the study. The data suggest that retaliatory action by the worker against the supervisor is short livedvanishing after about two hours. Being a target of incivility from a supervisor seems to alter a target's mood for only a few hours. This suggests that a buildup of animosity between subordinates and supervisors is more likely if contact is frequent rather than sporadic. In a finding that relates directly to the current proposed study, the research suggests that an individual's ability to control his/her emotions after being a target of incivility depends on the strengths of the self-regulating capacities of the individual. Specifically, exhaustion can be a result of experienced incivility, and if an individual is exhausted, self-regulatory capacities are lowered.

Taylor and Kluemper (2012) investigated the influence that employee perception of workplace stress might have on the instigation of workplace aggressions. They posit a positive relationship between job stress, perceptions of experienced workplace incivility and enacted aggression. Specific individual personality trait variables such as neuroticism, agreeableness, and conscientiousness moderate this relationship. These personality traits may determine whether experiencing workplace incivility leads to 
aggressive behaviors by individual employees. Drawing paired supervisor-subordinate participants $(N=507)$ from a broad range of industries, employees responded to questionnaires assessing their job stressors, experienced workplace incivility, and personality traits while the supervisors reported on their enacted aggression. In developing the hypotheses to test these relationships, the authors relied heavily on the Dollard-Miller model of aggression (DM; Dollard, Doob, Miller, Mowrer, \& Sears, 1939; Miller, Pedersen, Earleywine, \& Pollock, 2003) and related literature. Because they were particularly interested in exploring the causes of the escalation of coercive behavior from relatively mild interactions to intense aggressive behavior, they also drew from literature that conceptualizes a "tit-for-tat" response to perceived incivility, which may spiral into incidences of workplace aggression (Andersson \& Pearson, 1999). Offended employees might reciprocate to the perceived uncivil words or deeds in a manner that will lead to aggressive behavior. Taylor and Kluemper (2012) developed and tested a path model exploring these relationships. Their findings supported the conceptual positive relationship between negative workplace conditions, perceived incivility, and enacted aggression. Specifically, they found that experienced incivility has a positive association with enacted aggression, supporting the "tit-for-tat" model of employee interaction, but with low mean scores marking the relationship. They interpreted this as countering the common assumption about incivility spirals being the frequent norm. Escalation of aggression, they concluded, is the exception rather than the norm of experienced incivility. They also concluded that a work environment dominated by unclear or conflicting expectations generates higher levels of perceived incivility among employees and higher incidences of workplace aggression. This conclusion corroborates the findings 
of other researchers who posit a relationship between stressful and chaotic work environments and a variety of perceived mistreatments in the workplace (Einarsen, Raknes, \& Mathiesen, 1994; Roscigno, Lopez, \& Hodson, 2009). Furthermore, the authors found that the indirect effect of job stress (ambiguity, conflict) were contingent on employee personality. That is, individuals reacted differently to job stressors depending on their personality characteristics. Individuals with low agreeableness were more likely to perceive incivility and escalate their responses to enacted aggression (the tit-for-tat response). Employees with high neuroticism perceived more incivility and displayed more aggressive behavior.

It is almost a truism that work and information overload, leading to intensified feelings of time pressure, is one of the primary causes of the rising rates of workplace incivility (Pearson et al., 2000). Blau and Andersson (2005) were the first to report a link between exhaustion and instigated incivility. Since then, others have provided support for this relationship (Van Jaarsveld et al., 2010, Walker et al., 2014). Moore (2000, p.336) defined work exhaustion as the "depletion of emotional and mental energy needed to meet job demands." Emotional exhaustion refers to the feeling that one's emotional and physical resources are depleted (Maslach, 1982). This phenomenon has been most studied in the service industries where, to cope with emotional exhaustion, employees distance themselves from their clients and other service recipients. Through interviews in the service industry, Maslach, Schaufeli, and Leiter (2001) learned that detachment as well as lack of concern led service providers to treat their clients in negative, callous, and dehumanizing ways. 
Blau and Andersson (2005) conducted one of the few studies exploring the factors associated with instigator motives in initiating workplace incivility and their relation to working conditions. They posited that instigated incivility is a distinct and underexplored construct. To establish the theoretical construct of instigated incivility, the authors used the Bennett and Robinson (2000) scale, which developed broad measures of workplace deviance. The study sample consisted of 232 working adults employed in a variety of organizations but accessed through the evening resource management classes offered by a northeastern U.S. university. They completed an adapted 7-item version of the Workplace Incivility Scale (WIS), and, because the WIS focuses on target perspectives, they adapted a version of the scale measuring instigated incivility. They also completed the 7-item interpersonal deviance scale mentioned above. The results weakly supported the hypothesis that instigated incivility is an independent construct from workplace deviance and experienced workplace incivility. Job satisfaction had a negative correlation with workplace incivility, and work exhaustion had a positive correlation with instigated workplace incivility.

Francis, Holmvall and O'Brien (2015) employed an experimental design in their study testing the overriding hypothesis that experiencing incivility will lead to instigating incivility. Given the ubiquitous nature of emails as the primary means of organizational communication in the modern workplace, the authors set out to design an experimental manipulation of the medium with the hopes of contributing to the literature on the general causes of incivility. The structural nature of email communications offers this type of interaction certain limitations and potentials for incivility research. The authors cite literature on the prevalence of email as a form of communication in office environments 
as well as its informal nature. The informality manifests itself in a variety of ways, but for their research they pointed out that an individual might get away with acting uncivilly in an email by blaming the medium's informal nature. Incivility in emails may manifest itself in a variety of subtle ways: condescending or belittling comments, inappropriately shared personal information.

Francis et al. (2015) hypothesized that an individuals will instigate more incivility when responding to uncivil rather than civil emails. The also hypothesized that an individuals with high workloads will instigate more incivility in emails than individuals with low workloads. Their third hypothesis expanded on the importance of workload by stating that workload will exacerbate the effect of receiving an uncivil email, so workers with high workload will instigate more uncivil responses relative to workers with low workloads. The study included 86 psychology third-year or higher undergraduate students at a small Canadian university. The students were under the assumption that they were participating in a study evaluating their managerial ability by how well they dealt with "in-basket" exercises, well established ways of measuring managerial potential. The participants were randomly assigned to workload groups (high vs low) and they responded to emails from subordinates varying in civility (civil vs uncivil). Participants responded more uncivilly to uncivil emails, and workload had an impact on the quantity and quality of uncivil responses to the initial stimuli. Thus, the importance of workload as a causal agent in instigating incivility was supported.

\section{Summary of the Literature}

Social dominance theory suggests that social dominators are not only likely to be racially prejudiced, but also that their prejudice is likely to be more sophisticated (Duriez 
\& Van Hiel, 2002). High SDO scorers often exhibit a style of thinking which reflects a form of modern racism that surfaces in subtle ways when it is safe, socially acceptable, and easy to rationalize (Dovidio \& Gaertner, 1997). Social power theory maintains that society, through social expectations and norms, gives certain individuals power over others (French, 1956). Incivility scholars suggest that manifestations of workplace incivility may function as a mean of asserting power (Cortina et al., 2017; Schilpzand et al., 2016). In the organizational context, this phenomenon translates into the more vulnerable position of employees with lower social or organizational power (Cortina et al., 2001). Individuals with more power have more opportunities to be uncivil and are more likely to get away with such behavior (Pearson et al., 2000; Pearson \& Porath, 2005).

Social psychologists have long recognized that prejudices are influenced by perceivers' emotional states (Dasgupta et al, 2009). Hence, like the flight attendants in Hochschild's (1983) study who understand that the emotional expectations of their job necessitate that they suppress, rather than express, their irritation with passengers, employees who harbor prejudice toward members of minority groups stifle, rather than express their feelings in the workplace (Grandey \& Gabriel, 2015). Demographic diversity may be a source of increased emotion regulation in work environments because organizations generally require employees to hide personal biases (Kim et al., 2013). Few studies have examined the relationship of SDO and emotions and the proponents of social dominance theory did not discuss its emotional correlates. One study investigating the relationship between SDO and the expression of emotions indicated that social dominators tend to withhold emotional expressions (Van Hiel et al., 2006). Recent 
findings on the two dimensions of SDO suggest that individuals who score high on SDO$\mathrm{D}$ are more likely to engage in overt and aggressive forms of behavior toward members of groups that are perceived as inferior (Ho et al., 2012; Ho et al., 2015). In contrast, individuals who score high on the SDO-E dimension are more likely to engage in covert and subtle forms of behavior toward groups that are perceived as inferior (Ho et al., 2012; Ho et al., 2015).

The emotion regulation strategy of cognitive reappraisal influences whether a particular emotion response tendency is triggered, and is therefore expected to have generally positive implications for affective and social functioning (Gross \& Thompson, 2007; Grandey, 2000; Gross, 1998b; Hochschild, 1983; Liu et al., 2010; Webb et al., 2012). In contrast, the expressive suppression strategy of emotion regulation influences the modulation of emotion response once it is triggered, and is expected to have a generally more negative implication for affective and social functioning (Gross, 1998b; Gross \& Levenson, 1993; Hochschild, 1983).

Andersson and Pearson (1999) seminal article that introduced the construct of workplace incivility to the management literature included a reference to workplace norms in defining workplace incivility - uncivil behavior is "in violation of workplace norms for mutual respect” (p. 457). Incivility researchers have identified workplace norms as a significant factor in the study of workplace incivility (Cortina et al., 2017; Schilpzand et al., 2016). The review of the literature suggests that relationships among the variables in this study may change in strength or direction based on norms of workgroup civility climate. 


\section{CHAPTER III}

\section{METHOD}

Chapter III begins by stating the purpose of the study followed by the research question and hypotheses, research design, population, sample size, variables and instrumentation, procedures, and the data analysis. Chapter III concludes with a summary of the methods discussed in the chapter.

\section{Purpose of the Study}

The purpose of this study was to develop and test a parallel mediator model of instigated incivility moderated by workgroup civility climate. The research project examined the mediation of the emotion regulation strategies of cognitive reappraisal and expressive suppression, on two types of social dominance orientation - support for intergroup dominance (SDO-D) and intergroup antiegalitarianism (SDO-E) - on the outcome of instigated incivility.

\section{Research Questions and Hypotheses}

Two research questions and four major hypotheses guided this study.

Research question 1: Do the emotion regulation strategies of cognitive reappraisal and expressive suppression mediate the relationship between the two types of social dominance orientation (SDO-D and SDO-E)? The following hypotheses were tested:

Hypothesis 1a: There is a statistically significant positive relationship between SDO-D and instigated incivility.

Hypothesis $1 b$ : There is a statistically significant positive relationship between SDO-E and instigated incivility. 
Hypothesis 2a: There is a statistically significant relationship between SDO-D and the mediators expressive suppression and cognitive reappraisal.

Hypothesis ${ }_{2 b}$ : There is a statistically significant relationship between SDO-E and the mediators expressive suppression and cognitive reappraisal.

Hypothesis ${ }_{3 a}$ : Expressive suppression mediates the relationship between SDO-D and instigated incivility, while controlling for SDO-E.

Hypothesis ${ }_{3 b}$ : Cognitive reappraisal mediates the relationship between SDO-D and instigated incivility, while controlling for SDO-E.

Hypothesis 3c: Expressive suppression mediates the relationship between SDO-E and instigated incivility, while controlling for SDO-E.

Hypothesis 3d: Cognitive reappraisal mediates the relationship between SDO-E and instigated incivility, while controlling for SDO-D.

Research question 2: What are the moderating effects of workgroup civility climate on the proposed model of instigated incivility? The following hypothesis was tested:

Hypothesis 4: The paths of the model are moderated by workgroup civility climate such that the strength or direction of one or more paths are significantly different for subsamples from different civility climates.

\section{Research Design}

The study employed a non-experimental quantitative research design. Nonexperimental quantitative research designs are appropriate for assessing the strength and relationship among two or more variables and when making predictions where sample randomization or variable manipulation are not possible (Gall, Gall, \& Borg, 2005; 
Johnson, 2001; Pedhazur \& Schmelkin, 2013). Non-experimental quantitative designs tend to be strongest on measuring covariance and weakest on ruling out the influence of extraneous variables (Johnson, 2001). Non-experimental designs do not allow the making of causal statements (Field \& Hole, 2003). However, identification of strong correlations can serve to stimulate further research, including experiments, and to explore causal relationships (Creswell, 2014).

\section{Population}

The population for the present study consisted of individuals from a heterogeneous sampling of Mechanical Turk (MTurk) adult workers (18-years old or older), who were employed at least part-time (20 or more hours per week). The MTurk platform is the Amazon.com crowdsourcing technology that has increased in popularity among behavioral social scientists over the last several years. The term crowdsourcing has its origin in an article by Howe (2006), who defined it as a job outsourced to a group of people in the form of an open call. A benefit of the MTurk platform for behavioral research is that MTurk offers researchers access to a large sample of workers willing to do tasks - including participating in research studies - at a relatively low cost (Chan \& Holosko, 2016a; Mason \& Suri, 2012). Researchers have recruited participants via MTurk for a wide variety of research topics using a range of experimental, quasiexperimental, and survey designs, and in a variety of disciplines to include business and marketing (Whitla, 2009), education (Johnson \& Borden, 2012; Tinkler \& Woods, 2013), economics (Amir, Rand, \& Gal, 2012), social work (Chan \& Holosko, 2016b), health care (Turner, Kirchhoff, \& Capurro, 2012; Chunara et. al., 2012) and political science (Crawford \& Pilanski, 2012). 
Ipeirotis, Provost, and Wang (2010) present the most comprehensive analysis of MTurk workers and the data are updated multiple times during the day (see http://demographics.mturk-tracker.com/), with the most current characteristics of the millions of workers completing tasks on MTurk's crowdsourcing platform. These characteristics vary according to day and even hour, but the rate and range of change are measurable and consistent over time. The general characteristics of the MTurk workforce as of this writing are:

- Approximately $80 \%$ of the MTurk workforce is from the United States and $20 \%$ are from India.

- The gender participation of MTurk workers in the U.S. is balanced with 50\% male workers and $50 \%$ female workers.

- Roughly $40 \%$ of MTurk workers in the U.S. are born in the 1980 s, about $28 \%$ of workers are born in the 1990s, another 20\% are born in the 1970s, and 6\% are born in the 1960s.

- The median yearly household income of MTurk workers in the U.S. is approximately $\$ 50,000$, which is comparable to the median U.S. household income ( $\$ 53,657$ according to the 2015 ACS data).

The dominant concern in the methodological literature on the use of MTurk as a sample gathering technique is the concern over the characteristics of the population sample and their impact on the research results. Recent research on the demographic characteristics of MTurk workers compare the MTurk sample with other, more conventional samples. Respondents on MTurk tend to be younger (about 30 years old) than the general population, are more educated, underemployed, less religious, and more 
liberal than the general population (Paolacci \& Chandler, 2014). Within the United States, Asians are overrepresented, and Blacks and Hispanics are underrepresented on MTurk relative to the population as a whole (Berinsky, Huber, \& Lenz, 2012; Paolacci, Chandler, \& Iperotis, 2010; Shapiro, Chandler, \& Mueller, 2013). However, respondents recruited via MTurk are often more representative of the U.S. population than in-person convenience samples (Berinsky et al., 2012).

To determine the key demographic characteristics of MTurk panels with the national population, Huff and Tingley (2015) established a comparison measure, the Comparative Congressional Election Survey (CCES), which is a nationally stratified sample survey administered yearly. The survey questions measured the respondents' demographic and political characteristics. The authors compared the characteristics of the pools and conclude that both surveys - the MTurk survey $(N=2706)$ and the CCES survey $(N=1300)$ - have their strengths and weaknesses. The MTurk sample was younger than the CCES sample, was equally "white" racially, but included more males than the CCES sample. The MTurk platform was also more effective in attracting Hispanic and Asian respondents while lagging behind the CCES in attracting AfricanAmerican respondents. The two samples also were similar in the occupation of the members as well as the urban/rural continuum of participation. The data comparisons, as well as the methodological contributions testing external validity are promising for the research possibilities of MTurk (Huff \& Tingley, 2015).

The major limitations of all internet-based survey research include sampling and implementation issues (Dillman, 2000; Wright, 2005). The MTurk samples may not be representative of many specified populations as it is impossible to calculate a 
respondent's chance of being sampled because MTurk workers are individuals who opt into MTurk and choose to accept a task or survey, (Baker et al., 2010). However, numerous researchers support the view that samples drawn using the MTurk platform provide quality data when compared to other convenience sample methods (Bartneck, Duenser, Moltchanova, \& Zawieska, 2015; Gosling \& Mason, 2015; Keith \& Harms, 2016; Rouse, 2015). Researchers support the assertion that samples drawn using MTurk are reliable on a variety of dimensions. Behavioral science scholars have found that MTurk samples offer psychometric validity for the assessment of individual differences (Buhrmester et al., 2011; Shapiro et al., 2013), and that the linguistic judgments of MTurk samples are comparable to college student samples (Sprouse, 2011). Economists using MTurk samples to test economic games found that MTurk samples perform at the same level as traditional participants. (Amir et al., 2012; Goodman, Cryder, \& Cheema, 2013; Horton, Rand, \& Zeckhauser, 2011; Paolacci et al., 2010). The MTurk samples have a great potential for organizational researchers (Landers \& Behrend, 2015). In the study of workplace organizations, designed to compare the external validity of different types of convenience samples, Landers and Behrend (2015) concluded that MTurk samples are more diverse than traditional convenience samples and more controllable in terms of setting the predictable parameters of the study population.

\section{Sample Size}

Sample size is an important consideration for making inferences regarding the characteristics of the population from measures of the sample (Hinkle, Wiersma, \& Jurs, 2006). A sample size of 5 to 50 participants per variable is recommended for regression analysis methods (Green, 1991). A power analysis of .80 with an effect size of .15 and 
alpha of .05 indicates a sample size of 120 to be appropriate (Hinkle et al., 2006). However, the present study involved a structural equation modeling (SEM) approach, a larger sample size was required to maintain statistical power and obtain stable parameter estimates and standard errors (Schumacker \& Lomax, 2010). O'Rourke, Psych, and Hatcher (2013) suggest that the minimum sample size for SEM analyses should be at least 100 or five times the number of observed variables in the SEM model. As the maximum possible number of observed variables in the present model is 46 , the sample size for this analysis should be at least 230 participants. The researcher collected data from a sample of $(N=1639)$ MTurk workers.

\section{Variables and Instrumentation}

The study included a self-report survey battery of five scales and a demographic questionnaire. The following scales were used in this study: the Social Dominance Orientation Scale (SDO7; Ho et al., 2015), the Emotion Regulation Questionnaire (ERQ; Gross \& John, 2003), an adaptation of the Uncivil Workplace Behavior Questionnaire Instigated (UWBQ-I; Grey et al., 2016); the Civility Norms Questionnaire - Brief (CNQB; Walsh et al., 2012), and the short form of the Marlowe-Crowne Social Desirability Scale (Reynolds, 1972). Full versions of each measure and the demographic questionnaire are found in the Appendix. The next section presents a detailed description of the variables and their corresponding measures.

\section{Independent Variables - Predictors}

The study included two predictor variables representing the two dimensions of social dominance orientation - Intergroup Dominance (SDO-D) and Intergroup 
Antiegalitarianism (SDO-E) - measured with the Social Dominance Orientation Scale (SDO7; Ho et al., 2015).

Social dominance orientation scale (SDO7). The 16-item measure of Social Dominance Orientation (SDO7; Ho et al., 2015) measures the two dimensions of social dominance orientation, SDO-D and SDO-E. Individuals who score high on SDO-D are oriented toward overt forms of domination toward others, while those who score high on SDO-E engage in subtle means of hierarchy maintenance (Ho et al., 2015). Respondents were required to rank each statement from 1 to 7 on a Likert scale, with 1 being "strongly oppose" and 7 "strongly favor." The scale consists of Pro-Trait items (negatively worded) and Con-Trait items (positively worded). The Con-Trait items are reverse scored so that high scores indicated high levels of social dominance orientation in both the SDOD and SDO-E dimensions. Ho and colleagues (2015) maintain that negatively worded and positively worded items minimize the potential of social desirability bias in the scale. The following two sections detail the two specific dimension of social dominance assessed in this study.

$\boldsymbol{S D O}-\boldsymbol{D}$. Intergroup dominance (SDO-D) is characterized by overt forms of oppression and aggressive behavior that is intended to maintain the subordination of minority groups (Ho et al., 2015). The 8-item SDO-D subscale includes statements such as "Some groups of people are just more worthy than others" and "Inferior groups should stay in their place." The SDO-D subscale reported reliability estimates between .89 and .82 across seven samples (Ho et al., 2015). The reliability estimate for the scale is the study was .93 . 
SDO-E. Intergroup antiegalitarianism (SDO-E) involves the maintenance of intergroup inequalities that are achieved via subtle hierarchy-enhancing ideologies and social policies (Ho et al., 2015). SDO-E items include statements such as "It would be good if all groups could be equal" and "No one group should dominate in society." The 8-item SDO-E subscale reported reliability estimates between .93 and .82 across seven samples (Ho et al., 2015). The reliability estimate for the scale is the study was .93.

\section{Independent Variables - Mediators}

This study included two mediator variables representing the emotion regulation strategies of cognitive reappraisal and expressive suppression, which were measured with the Emotion Regulation Questionnaire (ERQ; Gross \& John, 2003).

Emotion regulation questionnaire (ERQ). The 10-item ERQ scale to measures the habitual use of two emotion regulation strategies - cognitive reappraisal and expressive suppression (Gross \& John, 2003). Respondents indicated the extent to which they agree or disagree with each scale item on a 7-point Likert scale ranging from "strongly disagree" to "strongly agree." The correlation between cognitive reappraisal and expressive suppression is close to zero, indicating that individuals who use cognitive reappraisal are less likely to use expressive suppression as an emotion regulation strategy (Gross \& John, 2003). Thus, cognitive reappraisal and expressive suppression are two independent emotion regulation strategies that different individuals use to varying degrees. The cognitive reappraisal and expressive suppression are also distinct from measures of intelligence and measures of the Big Five personality trait dimensions (John, Naumann, \& Soto, 2008). The structure of the ERQ is consistent across samples, ages, and cultures, indicating a clear two-factor solution that was confirmed among multiple 
samples of younger and older adult populations in the United States (John \& Gross, 2004), Germany (Abler \& Kessler, 2009), Italy (Balzarotti, John, \& Gross, 2010), China (English \& John, 2013), and more than 15 other language communities (Matsumoto et al., 2008).

Cognitive reappraisal. The cognitive reappraisal subscale includes 6 items asking participants how they typically deal with negative emotions they experience. Sample items include, "I control my emotions by changing the way I think about the situation I'm in" and "When I want to feel more positive emotions (such as joy or amusement), I change what I'm thinking about." The scale has reported reliability estimates between .79 and .82 (Gross \& John, 2003; Wiltink et al., 2011). The scale is kept continuous with higher scores indicating an emotion regulation strategy of cognitive reappraisal. The reliability estimate for the scale is the study was .91 .

Expressive suppression. The expressive suppression subscale consists of 4 items asking participants questions such as, "I keep my emotions to myself" and "When I am feeling negative emotions, I make sure not to express them." The scale has reported reliability estimates between .79 and .82 (Gross \& John, 2003; Wiltink et al., 2011). The scale is kept continuous with higher scores indicating an emotion regulation strategy of expressive suppression. The reliability estimate for the scale is the study was .86 .

\section{Independent Variable - Moderator}

The study included one moderator variable of workgroup civility climate, measured with the Civility Norms Questionnaire (CNQ-B; Walsh et al., 2012).

Civility Norms Questionnaire - Brief (CNQ-B). The CNQ-B measures norms of workgroup civility climate by assessing the perception employees have of the norms 
supporting respectful behavior among workgroup members (Walsh et al., 2012). The CNQ-B is a 4-item measure on a 7-point Likert scale ranging from 1 "strongly disagree" to 7 "strongly agree". Items on the CHQ-B include statements such as "rude behavior is not accepted by your coworkers," and "respectful treatment is the norm in your unit/workgroup." Higher scores indicate a more positive civility climate within the workgroup. Reliability estimates for this scale range from .85 to .78 (Walsh et. al, 2012). The reliability estimate for the scale is the study was .84

\section{Independent Variable - Control}

The study included one control variable for social desirability bias measured with the Marlow-Crowne Social Desirability Scale- Short Form C (Reynolds, 1982). Addressing social desirability bias was important in this study due to the sensitive nature of asking individuals to report levels agreement on statements about group dominance and equality, and the frequency of instigated uncivil behavior in the workplace.

Marlow-Crowne Social Desirability Scale- Short Form C. Reynolds's (1982) 13-item social desirability scale measures the tendency of participants to portray themselves in a positive manner when testing situations address sensitive cultural norms (Crown \& Marlowe, 1960). Participants were asked to rate items as "true" or "false" (e.g., "I sometimes try to get even rather than forgive and forget"). The reliability coefficient reported for this scale has ranged from .55 to .67 in previous studies (Reio, 2010). The reliability estimate for the scale is this study was .81.

\section{Dependent Variable}

The dependent variable in this study was instigated workplace incivility. Instigated incivility was measured with an adaptation of the Uncivil Workplace Behavior 
Questionnaire (UWBQ-I; Gray et al., 2016). The UWBQ-I provides a comprehensive conceptualization of instigated incivility that includes the dimensions of hostility, privacy invasion, exclusionary behavior, and gossiping. For the purpose of the present study the lead in phrase was adapted to capture instigated incivility toward a coworker. Participants indicated how often during the past year they engaged in each of the activities toward a coworker while at work. The original scale does not specify the target of incivility. The scale included 20 items on a 4-point Likert scale ranging from "hardly ever (once every few months or less)" to "frequently" (at least once a day). The items in the scale were adapted to include a coworker frame of reference. The original items keep the target frame of reference open to "another", which may be interpreted as a subordinate or supervisor. The original UWBQ-I inventory assesses four dimensions of instigated incivility: privacy invasion (e.g., “open a co-worker's desk drawer without prior permission"), exclusionary behavior (e.g., "intentionally fail to pass on information which co-worker should be made aware of"), hostility (e.g., "raise your voice while speaking to a co-worker"), and gossiping (e.g., "talked about a co-worker behind his/her back"). The reliability estimates reported for the UWBQ-I subscales are .80 for hostility, .75 for privacy invasion, .86 for exclusionary behavior, and .88 for gossiping (Gray et al., 2017). The total reliability estimate for the scale is this study was .93 . The reliability estimates for the subscales were .87 for gossiping, .84 for hostility, and .90 for exclusionary behavior. The privacy invasion subscale was not included in the data analysis as the items reflect a traditional workplace setting with questions such as "open a co-worker's desk drawer without prior permission." The researcher decided to not include these items after reviewing the demographic characteristics of the sample, which 
indicated respondents worked in a variety of industries that do not typically include the traditional office setting.

\section{Demographic Variables}

Demographic variables were chosen to describe the sample as directed by the research literature (Cortina et al., 2017; Ho et al., 2015; Schilpzand et al., 2016; Sidanius \& Pratto, 1999). The demographic questionnaire asked respondents to describe themselves by the following categories: gender, race/ethnicity, country of birth, education, political affiliation, industry type, number of employees in organization, and supervisory role.

\section{Procedures}

An Internet-based self-report survey battery was used to collect data via Amazon's MTurk crowdsourcing platform. The advantages of Internet-based survey research include low cost, design flexibility, access to participants in distant locations, ability to reach participant who are difficult to contact, anonymity, time efficiency, and the convenience of an automated data collection process (Dillman, Smyth, \& Christian, 2009; Wright, 2005). Careful consideration was given to the methodological concerns associated with Internet-based survey research on MTurk. The following section discusses the methodological concerns regarding participant behavior on MTurk and the quality of data collected on MTurk.

\section{Behavior of Research Participants on MTurk}

Research methodologists that examined the use of the MTurk worker population in research studies indicate that the behavior of participants on MTurk is comparable to the behavior of traditional laboratory participants (Horton et al., 2011; Paolacci et al., 
2010). Mason and Watts (2010) used MTurk samples to study the effects of pay rate on output quantity and quality. Suri and Watts (2011), used the MTurk platform to study social conflicts and dilemmas over networks. Ho and colleagues (2015) included samples from MTurk to validate the social dominance scale that is used in the present study. A behavioral concern regarding the use of the MTurk platform for research studies is the attentiveness of the respondents to the task at hand. Chandler, Mueller \& Paolacci (2014) asked 300 MTurk respondents what they were doing while they completed a study, and found that $18 \%$ of responders were watching TV, $14 \%$ were listening to music, and $6 \%$ were communicating with others online. Some researchers have instituted "catch trials" to evaluate the diligence and attention of online responders. Catch trials consist of questions that the respondent must answer correctly to demonstrate they are paying attention to the task. Oppenheimer, Meyvis, and Davidenko (2009) and Hauser and Schwartz (2015) found that MTurk respondents fail catch trials at a lower rate than laboratory participants. Hauser and Schwartz (2015) found that lab-based participants failed catch trials at an outstanding $61 \%$ of the time, while MTurk respondents failed only $5 \%$ of the time. This suggests that the issue of distraction is not a large problem.

The researcher included three catch trials in the current study to identify unengaged responses. The catch trials in the current study included questions such as "If you are paying attention the correct answer to this question is "strongly agree." The study included an additional criterion to identify unengaged responses. The time it took participants to complete the survey was evaluated on the basis of results obtained in a 
pilot study. Participants who completed the survey in less than four minutes were considered unengaged, thus posing a threat to integrity of the data.

\section{MTurk Data Quality}

Studies support the view that samples drawn using the MTurk platform provide quality data when compared to other convenience sample methods (Bartneck et al., 2015; Gosling \& Mason, 2015; Keith \& Harms, 2016; Rouse, 2015). Economists using MTurk workers as participants in economic games have evidence to show they perform at the same level as traditional participants (Amir et al., 2012; Goodman et al., 2013; Horton et al., 2011; Paolacci et al., 2010).

Compensation for survey responses improves performance and reduces random responses on tasks that require a factually correct answer (Aker, El-Haj, Albakour, \& Kruschwitz, 2012). On tasks that rely on subjective responses, as do most psychological studies, there is no relationship between payment and quality of responses (Buhrmester et al., 2011). In tasks that require subjective responses, language comprehension and the respondent's understanding of the task are more significant variables in collecting quality data because payment does not influence the effort associated with a true or false response (Goodman et al., 2013). Some evidence is presented by Litman, Robinson, and Rosenzweig (2014), however, indicating that the amount of payment also influences data quality.

\section{Common Method Bias}

Common method bias (CMB) occurs when the relationship between variables is inflated as a consequence of the use of self-report measures (Podsakoff et al., 2003; Richardson, Simmering, \& Sturman, 2009; Spector, 2006). Common method bias is a 
potential source of measurement error that poses a threat to the relationships found between measures of different constructs (Reio, 2010). To minimize CMB, the current study implemented the following procedures: (a) ensure confidentiality of the participants, (b) counterbalance the order of the questions, (c) inform participants that there is no preferred or correct answer (d) ensure that all responses require equal effort (Reio, 2010). The study also included a social desirability scale to further minimize the effect of CMB.

\section{Pilot Study}

Following the Tailored Design Method (Dillman et al., 2009), a pilot study with 15 participants similar to the MTurk population was conducted to assess the time it took to complete the survey and to help with the identification of any design or administration issues. A link to the online survey was sent to the participants. The participants suggested a minor adjustment regarding the font size of the questions and identified two punctuation errors. Participants completed the survey in 5 to 9 minutes.

\section{Sampling Procedures}

Permission to conduct the study was approved by the Florida International Institutional Review Board (IRB). The data collection for the study was managed through TurkPrime (Litman, Robinson, \& Abberbock, 2017), a service that expedites the data collection process and optimizes MTurk functionality for the needs of researchers. The TurkPrime organization did not have access to the data as the survey link was managed by the researcher. Participant responses remained available only to the researcher.

The MTurk participants received access to a HIT titled Workplace Behavior Study. Participants who accepted the HIT were presented with a description of the 
research, that included participant eligibility criteria (18 years or older and employed at least 20 hours per week), and a consent form before proceeding to the survey link. Once the survey was completed, a verification code and instructions for MTurk HIT credit were provided. Participants received a payment of $\$ 1.00$ for completing the full survey to improve performance and reduce random responses (Aker et al., 2012). The MTurk HIT for the study remained active until a total of 1,500 complete responses was reached.

\section{Data Analysis}

Structural equation modeling (SEM) was conducted to test the hypothesized model of instigated incivility. The SEM models offer the possibility of including both measured and latent variables to produce a measurement model (Schumacker \& Lomax, 2010). Two major reasons for employing a SEM model are greater recognition given to the validity and reliability of observed scores from measurement instruments, and the ability to analyze complex theoretical systems with a large number of model-fit indices (Schumacker \& Lomax, 2010). For example, traditional statistical analyses of scores in multiple regression and path analysis do not adjust for measurement error (Schumacker \& Lomax, 2010). Additionally, SEM allows for more accurate estimates of the effects of the mediation and moderation compared to other more traditional methods (Russel, Kahn, Spoth, \& Altmaier, 1998). First, the procedures for data screening are discussed, followed by multicollinearity, multivariate normality, construct reliability, convergent and discriminant validity, measurement model, mediation model, moderation models, and a summary of the chapter. 


\section{Data Screening}

The dataset was assessed for duplicate responses using the incidence of repeated IP addresses. Only first attempts were retained unless the first attempt did not provide full data and a second attempt did. The sample was assessed for ineligible participants, as indicated by responses to the question asking participants whether they work less than 20 hours per week. Those who worked less than 20 hours are inapplicable to the study and were removed. Participants with negative responses to any of the three catch trial questions were removed if they failed to respond to the attention check. Participants who took less than four minutes to complete the survey were removed, as it was unlikely they allocated sufficient time to consider their responses. Finally, participants with missing responses to one or more variables intended for use as observed variables in the model were removed, as full and complete data were required for the analysis.

\section{Multicollinearity}

Multicollinearity was examined through correlations tests between the study variables to determine if any of the variables were correlated. Correlated variables may produce biased statistical tests (Kline, 2005). A correlation of .85 or greater (which approximately corresponds to a variance inflation factor greater than 10.00) indicates that the variables are correlated to the point that multicollinearity is a concern (Stevens, 2009). Variables with correlation lower than $r=.85$ were retained using Kline's criterion (2005).

\section{Multivariate Normality}

The distributional assumption of multivariate normality was examined during the SEM analysis. Multivariate normality in SEM may be examined using Mardia's 
coefficient, a multivariate measure of kurtosis (Kline, 2005). A significant Mardia's coefficient, as indicated by a corresponding critical ratio greater than 1.96 in absolute value, indicates that the data are not normally distributed (Kline, 2005). However, significance tests for normality are sensitive to sample size, with larger samples being more likely to produce significant results compared to smaller samples (2009). Kurtosis values of 3.00 or less in magnitude were considered acceptable (Westfall \& Henning, 2013).

\section{Construct Reliability}

The internal consistencies of each scale were examined during SEM analysis to determine whether the scales are reliable measures. Scale reliability was evaluated using the recommendation of a reliability cutoff of .80 (Lance, Butts, \& Michels, 2006; Nunnally, 1978).

\section{Convergent and Discriminant Validity}

Convergent validity was examined through the measure of average variance extracted (AVE), to determine the latent variables were well explained by their observed indicators in relation to the amount of variance associated with measurement error (Hair, Black, Babin, \& Anderson, 2010). The AVE values for the latent variables in this study were calculated in Excel with Fornell and Larker's (1981) formula; AVE = sum of the squared factor loadings) / (sum of the squared factor loadings + sum of error variances. Convergent validity was determined using the threshold value of AVE > 0.5 (Hair et al., 2010).

Discriminant validity was tested with by comparing the AVE and the squared correlation $\left(r^{2}\right)$ between the variables; an $r^{2}<$ AVE for either one of the individual 
variable AVE values, suggests that the variables each have more error free variance than the common variance with other variables. (Fornell \& Larker, 1981). Thus, discriminant validity is verified when variables have a lower correlation within their own parent factor than with the variables outside their parent factor (Hair et al., 2010).

\section{Measurement Model}

Model testing was initiated with a pooled confirmatory factor analysis (CFA) for an overall assessment of the proposed model's fit using SPSS AMOS version 23.0. The measurement model included the latent variables SDO-D (eight indicators), SDO-E (eight indicators), cognitive reappraisal (CR; six indicators), expressive suppression (ES; four indicators), hostility (HOST; four indicators), exclusionary behavior (EB; seven indicators), and gossiping (GOS; four indicators). Instigated Incivility was a second-order latent variable comprised of HOST, EB, and GOS, which were covaried with the other latent variables in the model. A maximum likelihood estimation (MLE) procedure with 2000 bootstrapped samples was used for the SEM calculation.

The measurement model was evaluated by three absolute fit measures including chi-square, $\chi^{2} / d f$, and root mean square error of approximation (RMSEA); two relative fit measures, including the normed fit index (NFI) and the comparative fit index (CFI); and the parsimony normed fit index (PNFI; Hooper, Coughlan, \& Mullen., 2008; Jöreskog, 1993). The target value for $\chi^{2} / d f$ is 5 or less (Meyers, Gamst, \& Guarino, 2016). The RMSEA is a measure of the average value of the residuals between the observed correlation from the sample and expected model estimated for the population; when interpreting this $\chi^{2}$, values below .08 are acceptable and values below .06 are ideal (Schreiber, Stage, Nora, Barlow, \& King, 2006). The NFI assesses the model by 
comparing the $\chi^{2}$ value of the model to the $\chi^{2}$ of the null model, while CFI is a revision of this statistic that takes into account sample size (Hooper et al., 2008). Values for CFI and NFI range between 0 and 1, with 1 indicating perfect fit (Hooper et al., 2008). For the CFI and NFI, a value of .90 or greater indicates reasonable fit, though values of .95 or greater are the preferred cutoff in determining that the model is very well specified (Hooper et. al, 2008). The target value for PNFI is .50 or greater (Meyers et al., 2016).

Upon assessment of these fit statistics, the measurement model was not wellspecified and required improvement. Modification indices were calculated to determine potential covariances to the model's fit. Modifications with the highest index were considered first and improvements were guided by theory (Schumacker \& Lomax, 2010), thus each modification's influence on the model fit was weighed against its relevance to the conceptual meaning of the model. After implementing influential modifications that were theoretically justifiable, the model was reassessed for fit. The process required several iterations before the model became an accurate portrayal of the paths among study variables in the dataset. Once the model had been well-specified and fit statistics were satisfactory, mediation and moderation analyses took place. To understand the paths in the model before attempting to assess them for potential moderation effects, mediation was conducted first.

\section{Mediation Model}

To address the hypotheses associated with the first research question, a structural model was constructed from the measurement model with paths drawn from SDO-D and SDO-E to cognitive reappraisal, expressive suppression, and instigated incivility, and paths drawn from cognitive reappraisal and expressive suppression to instigated 
incivility. To control for social desirability bias, the total social desirability score was added to the model as an observed variable. Paths were drawn from social desirability to cognitive reappraisal, expressive suppression, and instigated incivility. Social desirability was covaried with SDO-D and SDO-E. A maximum likelihood estimation (MLE) procedure with 2000 bootstrapped samples was used for the SEM calculation.

The structural model was evaluated by three absolute fit measures including chisquare, $\chi^{2} / d f$, and root mean square error of approximation (RMSEA); two relative fit measures, including the normed fit index (NFI) and the comparative fit index (CFI); and the parsimony normed fit index (PNFI; Hooper et al., 2008; Jöreskog, 1993). The effect sizes of all the statistically significant paths were evaluated using Cohen's (1988) criteria for evaluating effect sizes. Path coefficients $< \pm .28$ are small effects; medium effects range from $\pm .28-.49$; and, large effects are greater than \pm .49 (Cohen, 1988).

To test mediation, Baron and Kenny's (1986) method and the Sobel test were considered. However, as Hayes (2009) posited, the Baron and Kenny method of mediation analysis is outdated, and both the Baron and Kenny method and the Sobel test have been replaced with more sophisticated methods of analysis though SEM. Chen and Hung (2016) recommended the use of AMOS to calculate overall indirect effects through bootstrapping, which was the method of mediation analysis used in this study. To implement this method of mediation testing, the researcher followed guidelines set in place by Hayes (2009). Calculation of the model on 2000 bootstrapped samples provided output for standardized estimates and significance levels for overall indirect effects. Upon completion of the mediation analyses, the researcher gained evidence to support or reject 
the presence of the overall indirect effects of expressive suppression and cognitive reappraisal, as well as the associated effect sizes (i.e., standardized estimates).

According to Macho and Ledermann (2009), as well as Rose, Chassin, and Presson (2000), indirect effects with more than one mediator consist of the sum of a subset of specific indirect effects. In the context of the current study, both of the indirect effects between the independent variables (SDO-D or SDO-E) and the dependent variable (instigated incivility) consist of a specific effect from each independent variable through the mediator variable (expressive suppression), or through the mediator variable (cognitive reappraisal), to act on the outcome of instigated incivility. Because the effect of either independent variable through both mediators simultaneously constitutes the indirect effect, testing specific indirect effects was done under the assumption that there was an overall significant indirect effect to assess (Macho \& Ledermann, 2009). Testing specific indirect effects against an insignificant overall indirect effect increases the possibility of a Type I error, meaning that any method of calculating specific indirect effects has the potential for false positives (Macho \& Ledermann, 2009). However, to understand the specific indirect effects, it is possible to model a direct effect as the product of the path to the mediator from the independent variable and the path from the mediator to the dependent variable (Arbuckle, 2014). Calculation of these specific indirect effects through a product term helps describe the inner workings of the overall indirect effects, whether significant or not. Specific indirect effects were calculated using the user defined estimand function in AMOS. A mediation estimand was created to produce confidence intervals and $p$ values for the four possible mediator paths. The user 
defined estimand was used to evaluate the specific indirect effects in the mediation SEM and to later evaluate the specific indirect effects in the moderator group structural models.

\section{Moderation Models}

To address the hypothesis associated with the second research question, two structural models were constructed representing the two groups of the moderator variable of workgroup civility climate. A median split of the total workgroup civility climate score was used to create a low workgroup civility climate SEM and a high workgroup civility climate SEM. Both moderator group SEM's were evaluated by three absolute fit measures including chi-square, $\chi^{2} / d f$, and root mean square error of approximation (RMSEA); two relative fit measures, including the normed fit index (NFI) and the comparative fit index (CFI); and the parsimony normed fit index (PNFI; Hooper et al., 2008; Jöreskog, 1993). Model fit comparisons for the two moderator group were assessed to determine the applicability of each moderator group on the specified SEM. A maximum likelihood estimation (MLE) procedure with 2000 bootstrapped samples was used for the SEM calculations. The effect sizes of all the significant paths were evaluated on the basis of Cohen's (1988) criteria for evaluating effect sizes. Specific indirect effects were calculated using the user defined estimand function in AMOS.

Moderation testing through the lens of SEM relies on differences in path strength

or direction between two or more subsamples (Rigdon, Schumacker, \& Wothke, 1998). Some methods of moderation analysis through SEM compare full model fit between moderator groups, and preliminary assessment of fit comparison is important in testing the study's hypotheses, the proposed method of moderation extends beyond the 
comparison of overall model fit statistics to focus on differences in the strength of each path within the model. Differences in paths strengths were calculated in Excel.

The Fisher's r-to-z transformation was the first step in testing path differences (Fisher, 1921). This transformation resulted in a $z$ score for each path coefficient, which is a normalized expression of the correlation coefficient, and follows the formula $z^{\prime}=$ $.5[\ln (1+r)-\ln (1-r)]$, where $r$ is the path's estimated strength and $z$ ' is the $z$ score. The $z$ scores resulting from each pair of corresponding paths (i.e., identical paths from different subsamples) were then compared by subtracting the $z$ value for any path in moderator SEM group 2 from the $z$ value for that same path in moderator SEM group 1. Finally, the difference in score was divided by the standard deviation of the difference between the two sample sizes.

The results of these computations were a series of one $z$ test statistic per comparison. By assessing the $\mathrm{z}$ values for each path comparison in reference to a critical $z$, a series of $p$ values were available for interpretation. Following these procedures, the final $p$ values indicated whether any set of two paths were significantly different between the two moderator groups of workgroup civility climate. For significantly different paths, standardized estimates were compared to express the difference in effect size for each path's difference depending on the different subsamples.

By comparing paths between the models with different data, the researcher can detect differences between the different groups defined by a moderator variable. Paths that were not significant in the mediation model may not be meaningful to the moderation analysis. However, paths that were nonsignificant for one group of a moderator and 
significant for the other were compared as those differences retain meaning for the effects of different workgroup civility climates on instigated incivility.

\section{Summary of the Chapter}

Chapter III presented the research process for the proposed study to include the purpose of the study followed by the research question and hypotheses, research design, population, sample size, variables and instrumentation, procedures, and the data analysis. The next chapter presents the results of the data analysis. 


\section{CHAPTER IV}

\section{RESULTS}

The study examined a hypothesized parallel mediator model of instigated incivility using structural equation modelling (SEM). The model hypothesized that the relationship between the dimensions of social dominance (SDO-D and SDO-E) and instigated incivility would be mediated by the emotion regulation strategies of expressive suppression and cognitive reappraisal. Model paths were tested for the moderating effects of workgroup civility climate. Data cleaning results are presented first, followed by the background of the sample and multicollinearity tests. Second, the measurement model with multivariate normality, construct reliability, and convergent and discriminant validity results are presented. Third, the mediation model with testing of $H_{1 a}$ to $H_{3 d}$ and additional findings are presented. Finally, the moderation models with the testing of $H_{4}$ and summary of the results are presented.

\section{Data Cleaning}

There were a total of $(N=1639)$ responses collected for the study. The data were assessed for duplicate responses on repeated IP addresses. Only first attempts to complete the survey were retained unless the first attempt did not provide complete data and a second attempt did. Two hundred and thirty-three duplicate responses were removed. Next, the data were screened for ineligible respondents (i.e., respondents who reported working less than 20 hours per week). Thirty-five ineligible respondents were removed. The data were screened for unengaged responses using three attention check questions. One hundred responses were removed for failing one or more attention checks. An additional 232 responses were removed for completing the survey in less than four 
minutes. The remaining responses were screened for missing data. Twelve responses were removed for having incomplete data. The final sample was $N=1027$.

\section{Background of the Sample}

The demographic characteristics of the final sample are displayed in Table 1. Participants ranged in age from 18 to 86 years old $(M=36.30, S D=11.12)$. A slight majority of participants identified as White $73.2 \%(n=752)$. A large majority of participants were born in the United States $(n=969,94.4 \%)$. The largest proportion of participants had earned a bachelor's degree $(n=412,40.1 \%)$, and the most commonly reported political affiliation was Democrat $(n=458,44.6 \%)$. The most commonly reported industry of employment was "other" $(n=265,25.8 \%)$, followed by retail $(n=$ $154,15.0 \%)$ and education $(n=121,11.8 \%)$. The largest proportion of participants reported having 250 or more employees in their workplace $(n=312,30.4 \%)$. Finally, a slight majority of participants indicated that they did not have a supervisory role $(n=530$,

\section{$51.6 \%)$}

Table 1

Frequency Table of Demographic Variables $(N=1027)$

\begin{tabular}{lcc}
\hline Variable & Frequency & Percent \\
\hline Gender & & \\
Male & 548 & 53.4 \\
Female & 474 & 46.2 \\
Non-binary/third gender & 5 & 0.5 \\
& & \\
Race/Ethnicity & & \\
American Indian & 3 & 0.3 \\
Asian & 81 & 7.9 \\
Black & 61 & 5.9 \\
African American & 40 & 3.9 \\
Native American & 22 & 2.1 \\
\hline
\end{tabular}




\begin{tabular}{|c|c|c|}
\hline Pacific Islander & 1 & 0.1 \\
\hline Caucasian/White & 752 & 73.2 \\
\hline Hispanic/Latino & 67 & 6.5 \\
\hline \multicolumn{3}{|l|}{ Country of Birth } \\
\hline Argentina & 1 & 0.1 \\
\hline Armenia & 1 & 0.1 \\
\hline Bangladesh & 1 & 0.1 \\
\hline Bulgaria & 2 & 0.2 \\
\hline Cambodia & 1 & 0.1 \\
\hline Canada & 2 & 0.2 \\
\hline China & 1 & 0.1 \\
\hline Fiji & 1 & 0.1 \\
\hline Germany & 2 & 0.2 \\
\hline Guyana & 2 & 0.2 \\
\hline India & 4 & 0.4 \\
\hline Israel & 1 & 0.1 \\
\hline Jamaica & 2 & 0.2 \\
\hline Liberia & 1 & 0.1 \\
\hline Mexico & 2 & 0.2 \\
\hline Nepal & 1 & 0.1 \\
\hline New Zealand & 1 & 0.1 \\
\hline Other (Europe) & 1 & 0.1 \\
\hline Pakistan & 2 & 0.2 \\
\hline Peru & 2 & 0.2 \\
\hline Philippines & 6 & 0.6 \\
\hline Puerto Rico & 1 & 0.1 \\
\hline Singapore & 1 & 0.1 \\
\hline South Korea & 2 & 0.2 \\
\hline United Kingdom & 1 & 0.1 \\
\hline United States & 969 & 94.4 \\
\hline Vietnam & 1 & 0.1 \\
\hline Invalid response & 15 & 1.5 \\
\hline \multicolumn{3}{|l|}{ Highest Level of Education Completed } \\
\hline Less than a high school diploma & 6 & 0.6 \\
\hline High school degree or equivalent (e.g. GED) & 118 & 11.5 \\
\hline Some college, no degree & 243 & 23.7 \\
\hline Associate degree (e.g. AA, AS) & 118 & 11.5 \\
\hline Bachelor's degree (e.g. BA, BS) & 412 & 40.1 \\
\hline Master's degree (e.g. MA, MS) & 110 & 10.7 \\
\hline Professional degree (e.g. MD, DDS, DVM) & 11 & 1.1 \\
\hline Doctorate (e.g. PhD, EdD) & 9 & 0.9 \\
\hline
\end{tabular}




\begin{tabular}{lcc} 
Political Affiliation & & \\
Democrat & 458 & 44.6 \\
Republican & 219 & 21.3 \\
Independent & 333 & 32.4 \\
Rather not say & 17 & 1.7 \\
& & \\
Industry & & \\
Healthcare & 99 & 9.6 \\
Banking & 47 & 4.6 \\
Education & 121 & 11.8 \\
Manufacturing & 98 & 9.5 \\
Construction & 24 & 2.3 \\
Retail & 154 & 15.0 \\
Transportation & 28 & 2.7 \\
Arts and Entertainment & 69 & 6.7 \\
Real Estate & 21 & 2.0 \\
Accommodation and Food Service & 62 & 6.0 \\
Legal Services & 21 & 2.0 \\
Armed Forces & 2 & 0.2 \\
Federal Government & 16 & 1.6 \\
Other & 265 & 25.8 \\
Number of Employees & & \\
1 - 9 & & \\
$10-24$ & 130 & 12.7 \\
$25-99$ & 176 & 17.1 \\
100-249 & 238 & 23.2 \\
250+ & 171 & 16.7 \\
Supervisory Role & 312 & 30.4 \\
Yes & & \\
No & 497 & 48.4 \\
\hline
\end{tabular}

\section{Multicollinearity Tests}

Data were examined through zero-order correlations in SPSS to assess for multicollinearity among the latent constructs. No correlations exceeded .85, indicating that multicollinearity did not pose a concern for the structural analysis (Kline, 2005). Table 2 presents the zero-order correlations among the research variables. 
Table 2

Zero-Order Correlations among the Research Variables

\begin{tabular}{lccccccc}
\hline Variables & SDO-D & SDO-E & CR & ES & II & SD & WCC \\
\hline SDO-D & - & & & & & & \\
SDO-E & $.77 * *$ & - & & & & & \\
CR & $-.11 * *$ & $-.14^{* *}$ & - & & & & \\
ES & $.18^{* *}$ & $.16^{* *}$ & $-.07 *$ & - & & & \\
II & $.37 * *$ & $.30^{* *}$ & $-.13 * *$ & $.18^{* *}$ & - & & \\
SD & $-.08 * *$ & $-.07 *$ & $.17 * *$ & .01 & $-.24 * *$ & - & - \\
WCC & $-.19 * *$ & $-.16^{* *}$ & $.32 * *$ & -.02 & $-.34 * *$ & $.26 * *$ & - \\
\hline
\end{tabular}

Note. $N=1027$. SDO-D = Intergroup Dominance; SDO-E = Intergroup

Antiegalitarianism; CR = Cognitive Reappraisal; ES = Expressive Suppression; II = Instigated Incivility; $\mathrm{SD}=$ Social Desirability; $\mathrm{WCC}=$ Workgroup Civility Climate . $* p<.05 ; * * p<.01$.

\section{Measurement Model}

The chi-square of the initial measurement model was significant, $\chi^{2}(766)=$ $3996.38, p<.001$, however the chi-square tests are affected by large sample sizes and non-normality in the distribution of the data (Kline, 1998). The initial measurement model demonstrated good fit for RMSEA (.06) and PNFI (.82), but not for $\chi 2 / d f(5.22)$, NFI (.87), and CFI (.89). Standardized item loadings and modification indices were examined to improve model fit. CR_1 had a low negative loading (-.30); this was the result of a survey programming error which resulted in incorrect response options appearing for the question. The item was removed from the model. Additionally, the following pairs of intra-scale item error terms had modification indices exceeding 100: CTD_1 with CTD_2, CTD_2 with CTD_4, PTA_1 with PTA_3, PTA_3 with CTA_1, and CTA_1 with CTA_3. These pairs of error terms were covaried in the revised model. The revised model demonstrated good fit for all fit indices, $\chi^{2}(722)=3126.25, p<.001$, $\chi 2 / d f=4.33, \mathrm{RMSEA}=.06, \mathrm{NFI}=.90, \mathrm{CFI}=.92, \mathrm{PNFI}=.83$. The chi-square test is 
affected by large sample size and non-normality in the distribution of the data (Kline, 1998). The final measurement model with standardized coefficients is displayed in Figure 2.

\section{Multivariate Normality Tests}

Multivariate normality was assessed in the measurement model. Mardia's coefficient was significant, with a critical ratio $=207.71$, indicating that the data were not multivariate normal. As Mardia's coefficient is sensitive to sample size, thus individual kurtosis values were examined (Table 3). Six observed variables had kurtosis values exceeding 3.00 in magnitude. To account for the violation of normality, bootstrapped estimates were interpreted in the structural analysis (Westfall \& Henning, 2013).

Table 3

Test of Multivariate Normality

\begin{tabular}{lcc}
\hline Observed Variable & Kurtosis & Critical Ratio \\
\hline EB_1 & 2.28 & 14.94 \\
EB_2 & 4.37 & 28.61 \\
EB_3 & 8.23 & 53.82 \\
EB_4 & 2.72 & 17.81 \\
EB_5 & 1.22 & 8.01 \\
EB_6 & 8.14 & 53.24 \\
EB_7 & 5.50 & 35.96 \\
HOST_1 & 15.02 \\
HOST_2 & 2.30 & 19.96 \\
HOST_3 & 3.05 & 17.20 \\
HOST_4 & 2.63 & -1.89 \\
GOS_1 & -0.29 & 43.58 \\
GOS_2 & 6.66 & 5.70 \\
GOS_3 & 0.87 & 0.39 \\
GOS_4 & 0.06 & 1.04 \\
ES_1 & 0.16 & -5.95 \\
ES_2 & -0.91 & -3.80 \\
ES_3 & -0.58 & -6.88 \\
ES_4 & -1.05 & -5.13 \\
\hline
\end{tabular}




\begin{tabular}{lcc}
\hline CR_2 & 1.35 & 8.83 \\
CR_3 & 1.40 & 9.15 \\
CR_4 & 1.35 & 8.85 \\
CR_5 & 1.18 & 7.75 \\
CR_6 & 1.40 & 9.15 \\
CTA_1 & 2.76 & 18.03 \\
CTA_2 & 0.89 & 5.81 \\
CTA_3 & 1.41 & 9.20 \\
CTA_4 & 0.48 & 3.13 \\
PTA_1 & -0.25 & -1.61 \\
PTA_2 & -0.69 & -4.50 \\
PTA_3 & -0.42 & -2.74 \\
PTA_4 & -1.02 & -6.67 \\
PTD_1 & -0.57 & -3.74 \\
PTD_2 & -0.80 & -5.26 \\
PTD_3 & -0.86 & -5.65 \\
PTD_4 & -0.26 & -1.67 \\
CTD_1 & 1.08 & 7.07 \\
CTD_2 & 1.30 & 8.48 \\
CTD_3 & 2.07 & 13.56 \\
CTD_4 & 0.45 & 2.95 \\
Multivariate (Mardia's coefficient) & 751.41 & 207.71 \\
\hline
\end{tabular}

\section{Construct Reliability Tests}

Standardized loadings and reliability coefficients (i.e., Cronbach's alpha) are displayed in Table 4. All reliability coefficients exceeded .80, indicating good reliability for all measures (Nunnally, 1978).

Table 4

Standardized Loadings and Reliability Coefficients for Measurement Model

\begin{tabular}{llcc}
\hline Variable & Item & Cronbach's Alpha & Standardized Factor Loadings \\
\hline SDO-D & & .93 & \\
& & & .62 \\
& CTD_1 & & .71 \\
& CTD_2 & .53 \\
& CTD_3 & .67 \\
& CTD_4 & .87 \\
\hline
\end{tabular}




\begin{tabular}{|c|c|c|c|}
\hline & PTD_2 & & .95 \\
\hline & PTD_3 & & .90 \\
\hline & PTD_4 & & .88 \\
\hline \multirow[t]{9}{*}{ SDO-E } & & .93 & \\
\hline & PTA_1 & & .81 \\
\hline & PTA_2 & & .72 \\
\hline & PTA_3 & & .87 \\
\hline & PTA_4 & & .81 \\
\hline & CTA_1 & & .75 \\
\hline & CTA_2 & & .87 \\
\hline & CTA_3 & & .76 \\
\hline & CTA_4 & & .87 \\
\hline \multirow[t]{6}{*}{$\mathrm{CR}$} & & .91 & \\
\hline & CR_2 & & .73 \\
\hline & CR_3 & & .79 \\
\hline & CR_4 & & .84 \\
\hline & CR_5 & & .87 \\
\hline & CR_6 & & .87 \\
\hline \multirow[t]{5}{*}{$\mathrm{ES}$} & & .86 & \\
\hline & ES_1 & & .82 \\
\hline & ES_2 & & .74 \\
\hline & ES_3 & & .87 \\
\hline & ES_4 & & .70 \\
\hline Incivility & & .93 & \\
\hline \multirow[t]{5}{*}{ GOS } & & .87 & .69 \\
\hline & GOS_1 & & .64 \\
\hline & GOS_2 & & .80 \\
\hline & GOS_3 & & .86 \\
\hline & GOS_4 & & .87 \\
\hline \multirow[t]{5}{*}{ HOST } & & .84 & .81 \\
\hline & $\mathrm{HOST}_{-1}$ & & .85 \\
\hline & HOST_2 & & .79 \\
\hline & HOST_3 & & .85 \\
\hline & $\mathrm{HOST}_{4} 4$ & & .58 \\
\hline \multirow[t]{8}{*}{$\mathrm{EB}$} & & .90 & .90 \\
\hline & EB_1 & & .70 \\
\hline & EB_2 & & .71 \\
\hline & EB_3 & & .80 \\
\hline & EB_4 & & .75 \\
\hline & EB_5 & & .69 \\
\hline & EB_6 & & .84 \\
\hline & EB_7 & & .83 \\
\hline
\end{tabular}




\section{Convergent and Discriminant Validity Tests}

Tests of convergent validity indicated the average variance extracted (AVE) values for each of the latent variables in the measurement model met the AVE $>0.5$ threshold (Hair et al., 2010). The AVE values for the latent variables are presented in Table 5. Discriminant validity was determined by the results of the comparison between individual variable AVE values and the squared correlations of the research variables (Fornell \& Larker, 1981). Using Hair et al.'s criteria (2010), all variables had a lower correlation within their own parent factor than with the variables outside their parent factor. The AVE value (0.6) for SDO-D was at the cutoff point for establishing discriminant validity between SDO-D and SDO-E, however the AVE value (0.7) for SDO-E was higher than the SDO-D and SDO-E squared correlation $\left(r^{2}=.60\right)$.

Table 5

Average Variance Extracted and Interconstruct Squared Correlations

\begin{tabular}{|l|c|c|c|c|c|}
\hline Variables & AVE & SDO-D & SDO-E & CR & ES \\
\hline SDO-D & 0.6 & & & & \\
\hline SDO-E & 0.7 & .60 & & & \\
\hline CR & 0.7 & .01 & .02 & & \\
\hline ES & 0.6 & .05 & .03 & .01 & \\
\hline II & 0.6 & .22 & .10 & .10 & .05 \\
\hline
\end{tabular}

Note. AVE = Average Variance Extracted, SDO-D = Intergroup Dominance; SDO-E = Intergroup Antiegalitarianism; CR = Cognitive Reappraisal; ES = Expressive Suppression; II = Instigated Incivility. 


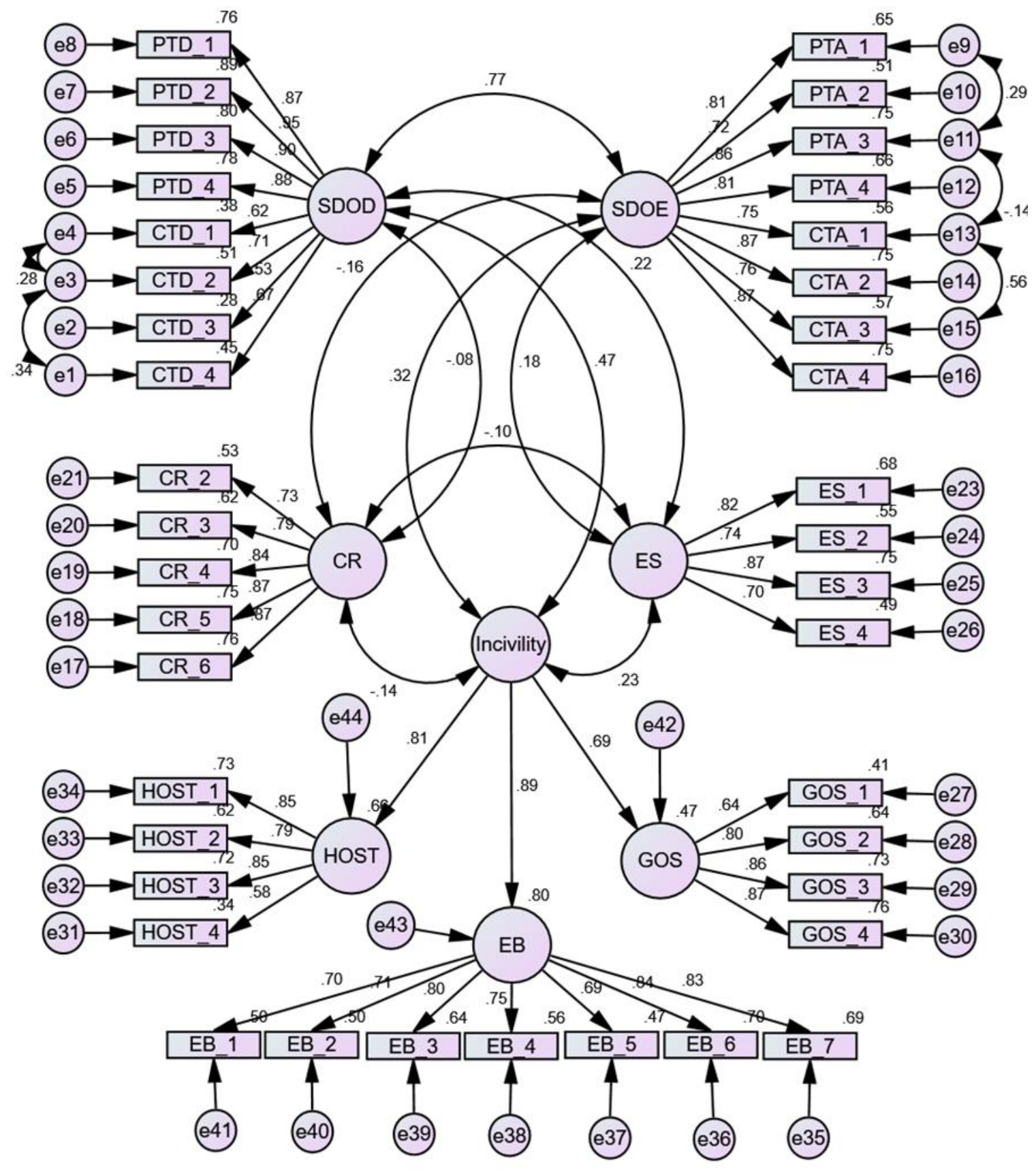

Figure 2. Measurement model with standardized coefficients. SDOD = Intergroup Dominance; $\mathrm{SDOE}=$ Intergroup Antiegalitarianism; SD = Social Desirability; $\mathrm{CR}=$ Cognitive Reappraisal; ES = Expressive Suppression; Incivility = Instigated Incivility; HOST = Hostility; EB = Exclusionary Behavior; GOS = Gossip. 


\section{The Mediation Model}

To address the hypotheses associated with the first research question, a SEM was constructed from the measurement model with paths drawn from SDO-D and SDO-E to cognitive reappraisal, expressive suppression, and instigated incivility, and paths drawn from cognitive reappraisal and expressive suppression to instigated incivility. To control for social desirability, the total social desirability score $(M=6.21, S D=3.46)$, was added to the model as an observed variable. Paths were drawn from social desirability to cognitive reappraisal, expressive suppression, and instigated incivility. Social desirability was covaried with SDO-D and SDO-E. The SEM mediation model of instigated incivility with standardized coefficients is displayed in Figure 3. The model demonstrated good fit for all fit indices, $\chi^{2}(758)=3286.15, p<.001, \chi 2 / d f=4.34, \mathrm{RMSEA}=.06, \mathrm{NFI}=.90$, $\mathrm{CFI}=.92, \mathrm{PNFI}=.83$. The chi-square test is affected by large sample size and nonnormality in the distribution of the data (Kline, 1998), but the other fit indices indicated an adequate fit (Kenney \& McCoach, 2003). The bootstrapped standardized coefficients are presented in Table 6. 
Table 6

Bootstrapped Standardized Path Coefficients for Structural Model

\begin{tabular}{|c|c|c|c|c|}
\hline Path & Bootstrapped $\beta$ & 95\% CI Lower & 95\% CI Upper & $\mathrm{p}$ \\
\hline $\mathrm{CR} \leftarrow \mathrm{SDO}-\mathrm{D}$ & .11 & -.01 & .23 & .080 \\
\hline $\mathrm{ES} \leftarrow \mathrm{SDO}-\mathrm{E}$ & .02 & -.10 & .13 & .752 \\
\hline $\mathrm{CR} \leftarrow \mathrm{SDO}-\mathrm{E}$ & -.23 & -.35 & -.11 & $* * .001$ \\
\hline $\mathrm{ES} \leftarrow \mathrm{SDO}-\mathrm{D}$ & .21 & .10 & .33 & $* * .002$ \\
\hline $\mathrm{CR} \leftarrow \mathrm{SD}$ & .18 & .11 & .24 & $* * .001$ \\
\hline $\mathrm{ES} \leftarrow \mathrm{SD}$ & .02 & -.05 & .09 & .563 \\
\hline Incivility $\leftarrow E S$ & .12 & .06 & .18 & $* * .001$ \\
\hline Incivility $\leftarrow \mathrm{CR}$ & -.07 & -.14 & .00 & $* .042$ \\
\hline Incivility $\leftarrow$ SDO-E & -.15 & -.27 & -.03 & $* .012$ \\
\hline Incivility $\leftarrow$ SDO-D & .54 & .43 & .66 & $* * .001$ \\
\hline Incivility $\leftarrow$ SD & -.18 & -.25 & -.13 & $* * .001$ \\
\hline \multicolumn{5}{|l|}{ Indirect Effects } \\
\hline Incivility $\leftarrow$ SDO-E & .02 & -.01 & .05 & .109 \\
\hline Incivility $\leftarrow$ SDO-D & .02 & -.01 & .05 & .130 \\
\hline
\end{tabular}

Note. $N=1027$. SDO-D = Intergroup Dominance; SDO-E = Intergroup

Antiegalitarianism; CR = Cognitive Reappraisal; ES = Expressive Suppression; Incivility $=$ Instigated Incivility; SD = Social Desirability. $\leftarrow=$ Path.

$* p<.05 ; * * p<.01$.

\section{Testing the Research Hypotheses $H_{1 a}$ to $H_{3 d}$}

The results from the testing of each hypothesis from $H_{1 a}$ to $H_{3 d}$ are presented next. The effect sizes of the significant paths were evaluated based on Cohen's (1988) criteria for evaluating effect sizes, thus path coefficients $< \pm .28$ are small effects; medium effects range from $\pm .28-.49$; and, large effects are greater than \pm .49 (Cohen, 1988).

Hypothesis $1_{a}$ posited a significant relationship between SDO-D and instigated incivility. Intergroup dominance (SDO-D) was significant and positively related to instigated incivility $(\beta=.54, p<.01)$ with a large effect size, supporting $\mathrm{H}_{1 \mathrm{a}}$. 
Hypothesis $1_{b}$ posited a significant relationship between SDO-E and instigated incivility. Intergroup Antiegalitarianism (SDO-E) was significant, but negatively related to instigated incivility $(\beta=-.15, p<.05)$ with a small effect size, the path coefficient was negative, thus not supporting $\mathrm{H}_{1 b}$.

Hypothesis $2_{a}$ posited a significant relationship between SDO-D and the mediators expressive suppression and cognitive reappraisal. Intergroup Dominance (SDO-D) was significant and positively related to expressive suppression $(\beta=.21, p<$ .05) with a small effect size, however, SDO-D was not significantly related to cognitive reappraisal $(\beta=.11, p>.05)$. These results partially support $\mathrm{H}_{2 \mathrm{a}}$.

Hypothesis $2_{b}$ posited a significant relationship between SDO-E and the mediators expressive suppression and cognitive reappraisal. Intergroup antiegalitarianism (SDO-E) was significant and negatively related to cognitive reappraisal $(\beta=-.23, p<.01)$ with a small effect size, however, SDO-E was not significantly related to expressive suppression $(\beta=.02, p>.05)$. These results partially support $\mathrm{H}_{2 \mathrm{~b}}$.

Hypotheses $3_{a}$ to $3_{d}$ posited specified four possible indirect paths from social dominance orientation (SDO-D and SDO-E) to instigated incivility. The results of the testing of $\mathrm{H}_{3 \mathrm{a}}$ to $\mathrm{H}_{3 \mathrm{~d}}$ are presented together for ease of understanding. There was no evidence for an indirect effect, meaning that the sum of the specific indirect effects through the mediators cognitive reappraisal and expressive suppression was not significant for the SDO-D and instigated incivility indirect path $(\beta=.02, p>.05)$, and not significant for the SDO-E to instigated incivility indirect path $(\beta=.02, p>.05)$. However, for completeness, the specific indirect effects of SDO-D and SDO-E on instigated incivility through each mediator $\left(\mathrm{H}_{3 a}\right.$ to $\left.\mathrm{H}_{3 d}\right)$ were examined further, though 
these results should be interpreted with caution, as the effect of repeated testing and evidence against an overall indirect effect lends to the possibility of Type I error (Lederman \& Macho, 2009).

For SDO-D, only the specific indirect effect on instigated incivility through expressive suppression was significant $(\beta=.01, p<.01)$, supporting $\mathrm{H}_{3 a}$; there was no evidence of an indirect path through cognitive reappraisal $(p>.05)$, not supporting $\mathrm{H}_{3 b}$.

For SDO-E, there was no evidence for a specific indirect effect on instigated incivility through expressive suppression $(p>.05)$, not supporting $\mathrm{H}_{3 c}$; the specific indirect effect on instigated incivility through cognitive reappraisal was significant $(\beta=.01, p<.05)$, supporting $\mathrm{H}_{3 d}$.

\section{Additional Findings}

The emotion regulation strategy of expressive suppression was significant and positively related to instigated incivility $(\beta=.12, p<.01)$, with a small effect size, in contrast the emotion regulation strategy of cognitive reappraisal was significant, but negatively related to instigated incivility $(\beta=-.07, p<.05)$, with a very small effect size. These results suggest that the use of expressive suppression increases instigated incivility, while the use of cognitive reappraisal reduces instigated incivility.

Social desirability had a significant and positive relationship with the emotion regulation strategy of cognitive reappraisal $(\beta=.18, p<.01)$, with a small effect size, while there was no evidence of a relationship between social desirability and the emotion regulation strategy of expressive suppression. 


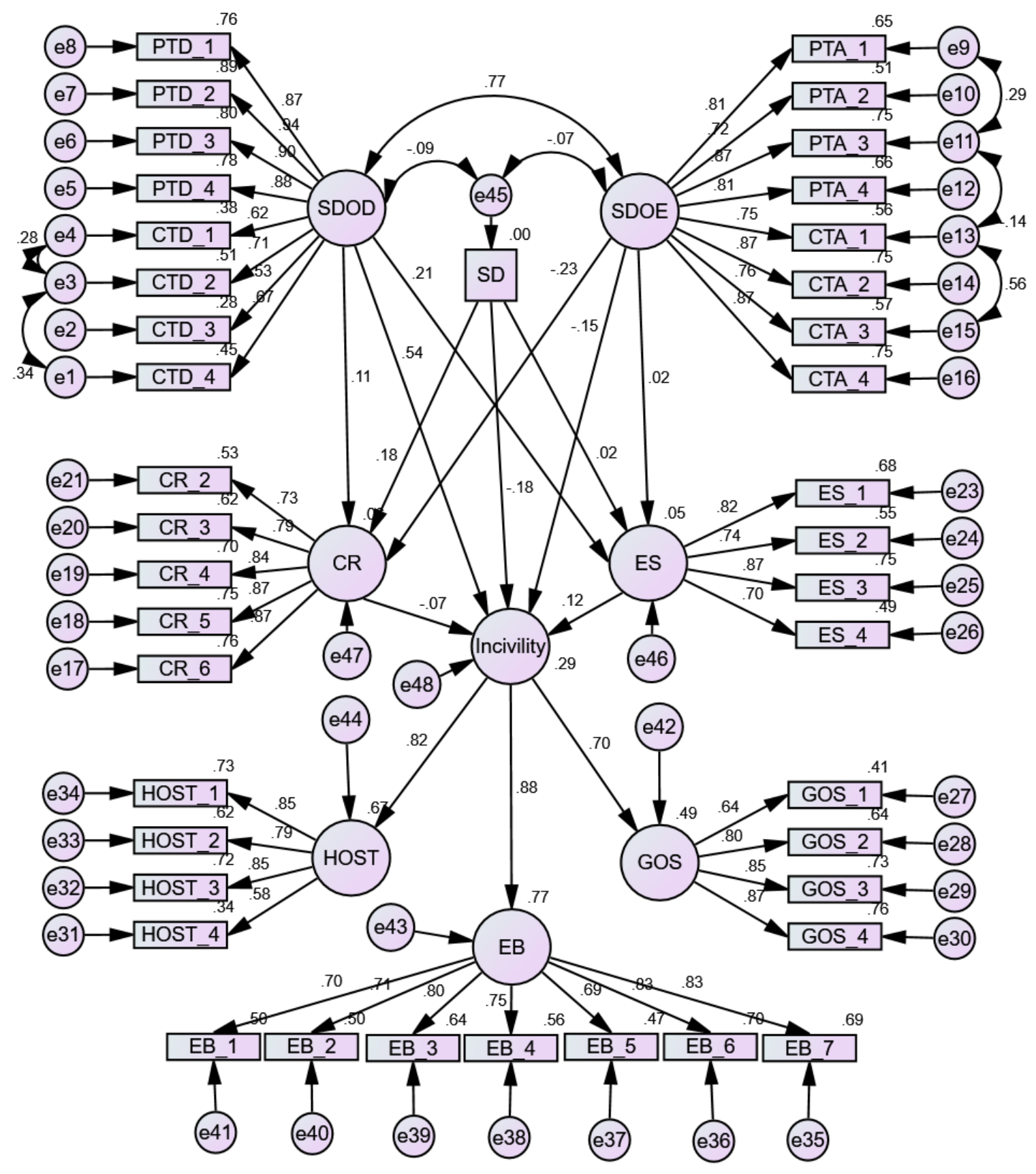

Figure 3. Mediation model with standardized coefficients. SDOD = Intergroup Dominance; $\mathrm{SDOE}=$ Intergroup Antiegalitarianism; $\mathrm{SD}=$ Social Desirability; $\mathrm{CR}=$ Cognitive Reappraisal; ES = Expressive Suppression; Incivility = Instigated Incivility; HOST $=$ Hostility; EB = Exclusionary Behavior; GOS = Gossip . 


\section{The Moderated Mediation Models}

The moderation hypothesis $H_{4}$, stated that the paths of the mediation model would be moderated by workgroup civility climate such that the strength or direction of one or more paths would be significantly different for subsamples from different civility climates. To address $H_{4}$, associated with the second research question, the paths in the previous SEM (Figure 3) were analyzed for group differences based on workgroup civility climate. Differences in path coefficients between groups (i.e., low vs. high civility climate) were tested for statistical significance for each group SEM. Figure 4 presents the SEM for the low workgroup civility climate group, and Figure 5 presents the SEM for the high workgroup civility climate group.

The model fit indices for the two subsamples for workgroup civility climate indicated that the low civility SEM had a slightly better overall model fit indices compared to the high civility SEM. However, in both models the NFI value was below the recommended value of .90 or higher (Hooper et al., 2008). Fit indices may degrade when models include a large number of variables as is the case in this study (Kenney \& McCoach, 2003). Because all other fit indices met the values for an acceptable fit, the models represent an acceptable fit to the data (Kenney \& McCoach, 2003). Table 7 provides a summary of the model fit indices for the two moderator groups of civility climate. 
Table 7

Model Fit Statistics for Low and High Workgroup Civility Climate Models

\begin{tabular}{lccccccc}
\hline Civility climate & $\chi^{2}(758)$ & $p$ & $\chi^{2} / d f$ & RMSEA & NFI & CFI & PNFI \\
\hline & & & & & & & \\
Low civility $(n=503)$ & 1904.57 & $<.001$ & 2.51 & .06 & .87 & .91 & .80 \\
High civility $(n=524)$ & 2491.22 & $<.001$ & 3.29 & .07 & .86 & .90 & .79 \\
\hline
\end{tabular}

Bootstrapped standardized path coefficients for both moderator groups were evaluated to identify significant paths (Table 8). These results are presented with the findings of the moderated mediation in the next section.

Table 8

Bootstrapped Standardized Path Coefficients for Moderator Groups

\begin{tabular}{|c|c|c|c|c|c|c|c|c|}
\hline \multirow[b]{3}{*}{ Path } & \multicolumn{4}{|c|}{ Low Civility Climate } & \multicolumn{4}{|c|}{ High Civility Climate } \\
\hline & \multirow[b]{2}{*}{$\beta$} & \multicolumn{2}{|c|}{$95 \% \mathrm{CI}$} & \multirow[b]{2}{*}{$p$} & \multirow[b]{2}{*}{$\beta$} & \multicolumn{2}{|c|}{$95 \% \mathrm{CI}$} & \multirow[b]{2}{*}{$p$} \\
\hline & & Lower & Upper & & & Lower & Upper & \\
\hline $\mathrm{CR} \leftarrow \mathrm{SDO}-\mathrm{D}$ & -.02 & -0.18 & 0.14 & .821 & .27 & 0.14 & 0.42 & **. .001 \\
\hline $\mathrm{ES} \leftarrow \mathrm{SDO}-\mathrm{E}$ & -.09 & -0.27 & 0.10 & .341 & .09 & -0.06 & 0.24 & .231 \\
\hline $\mathrm{CR} \leftarrow \mathrm{SDO}-\mathrm{E}$ & -.10 & -0.27 & 0.05 & .191 & -.32 & -0.49 & -0.17 & ${ }^{* *} .001$ \\
\hline $\mathrm{ES} \leftarrow \mathrm{SDO}-\mathrm{D}$ & .24 & 0.05 & 0.43 & $* .021$ & .18 & 0.03 & 0.33 & $* .021$ \\
\hline $\mathrm{CR} \leftarrow \mathrm{SD}$ & .12 & 0.02 & 0.21 & $* 011$ & .15 & 0.06 & 0.23 & $* * .001$ \\
\hline $\mathrm{ES} \leftarrow \mathrm{SD}$ & .04 & -0.06 & 0.15 & .461 & .02 & -0.09 & 0.11 & .761 \\
\hline Incivility $\leftarrow \mathrm{ES}$ & .12 & 0.04 & 0.21 & $* .011$ & .17 & 0.07 & 0.25 & ${ }^{* *} .001$ \\
\hline Incivility $<\mathrm{CR}$ & -.05 & -0.15 & 0.06 & .381 & -.01 & -0.09 & 0.06 & .771 \\
\hline Incivility $\leftarrow$ SDO-E & -.07 & -0.27 & 0.13 & .461 & -.25 & -0.43 & -0.10 & **.001 \\
\hline Incivility $<$ SDO-D & .50 & 0.32 & 0.69 & ${ }^{* *} .001$ & .53 & 0.38 & 0.69 & ${ }^{* *} .001$ \\
\hline Incivility $\leftarrow$ SD & -.11 & -0.21 & -0.02 & ${ }^{*} .011$ & -.16 & -0.23 & -0.09 & ${ }^{* *} .001$ \\
\hline \multicolumn{9}{|l|}{ Indirect Effects } \\
\hline Incivility $\leftarrow$ SDO-E & -0.01 & -0.04 & 0.03 & .588 & 0.02 & -0.02 & 0.06 & .281 \\
\hline Incivility $\leftarrow$ SDO-D & 0.03 & 0.01 & 0.07 & ${ }^{*} .027$ & 0.03 & -0.01 & 0.07 & .162 \\
\hline
\end{tabular}

Note. Low Civility $(N=503)$. High Civility $(N=524)$. SDO-D = Intergroup Dominance; SDO-E = Intergroup Antiegalitarianism; $\mathrm{CR}=$ Cognitive Reappraisal; ES = Expressive Suppression; Incivility = Instigated Incivility; SD = Social Desirability. $\leftarrow=$ Path. $* p<.05 ; * * p<.01$. 


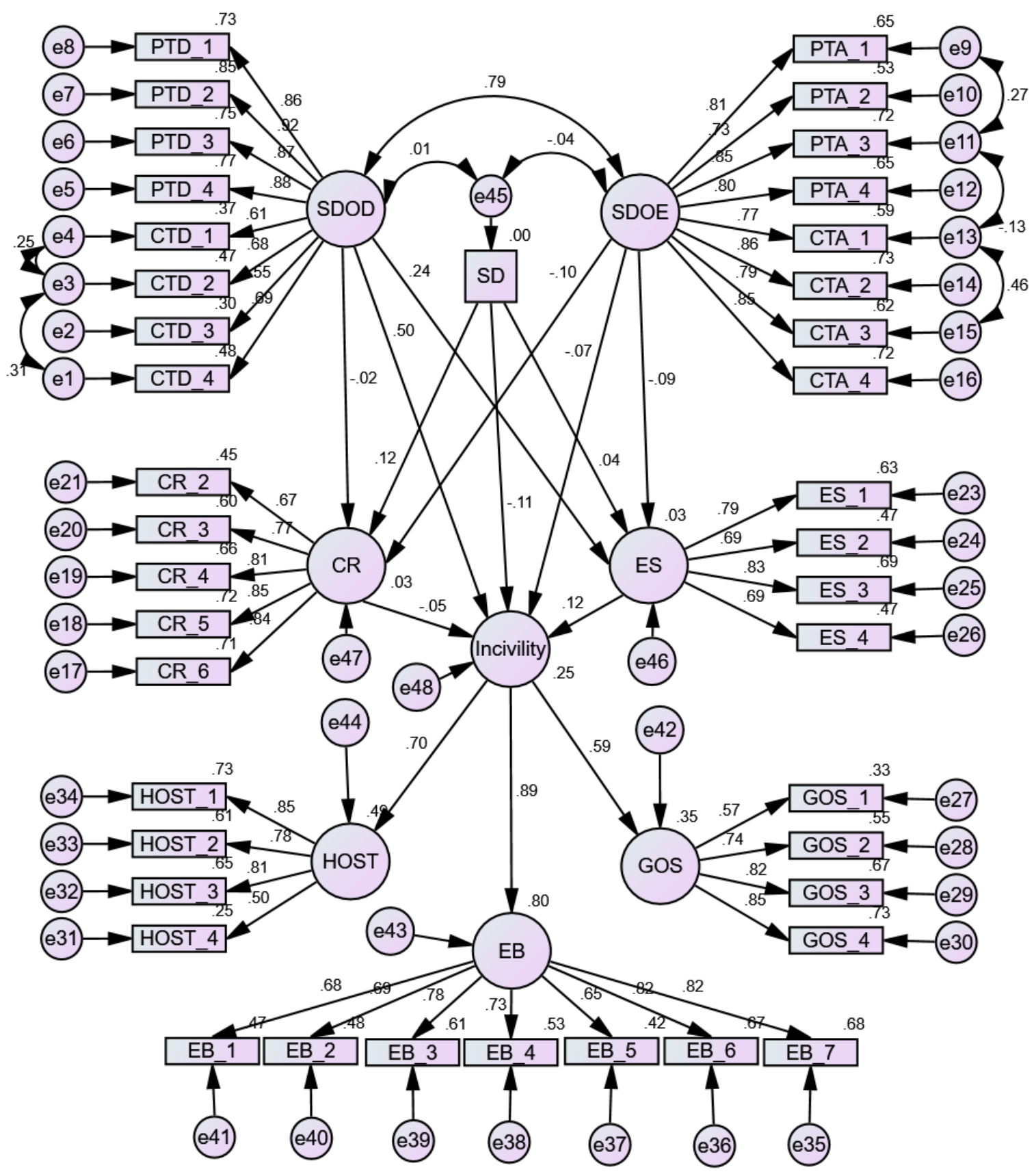

Figure 4. Low workgroup civility climate model with standardized coefficients. SDOD = Intergroup Dominance; SDOE = Intergroup Antiegalitarianism; SD = Social Desirability; $\mathrm{CR}=$ Cognitive Reappraisal; ES = Expressive Suppression; Incivility = Instigated Incivility; HOST = Hostility; EB = Exclusionary Behavior; GOS = Gossip. 


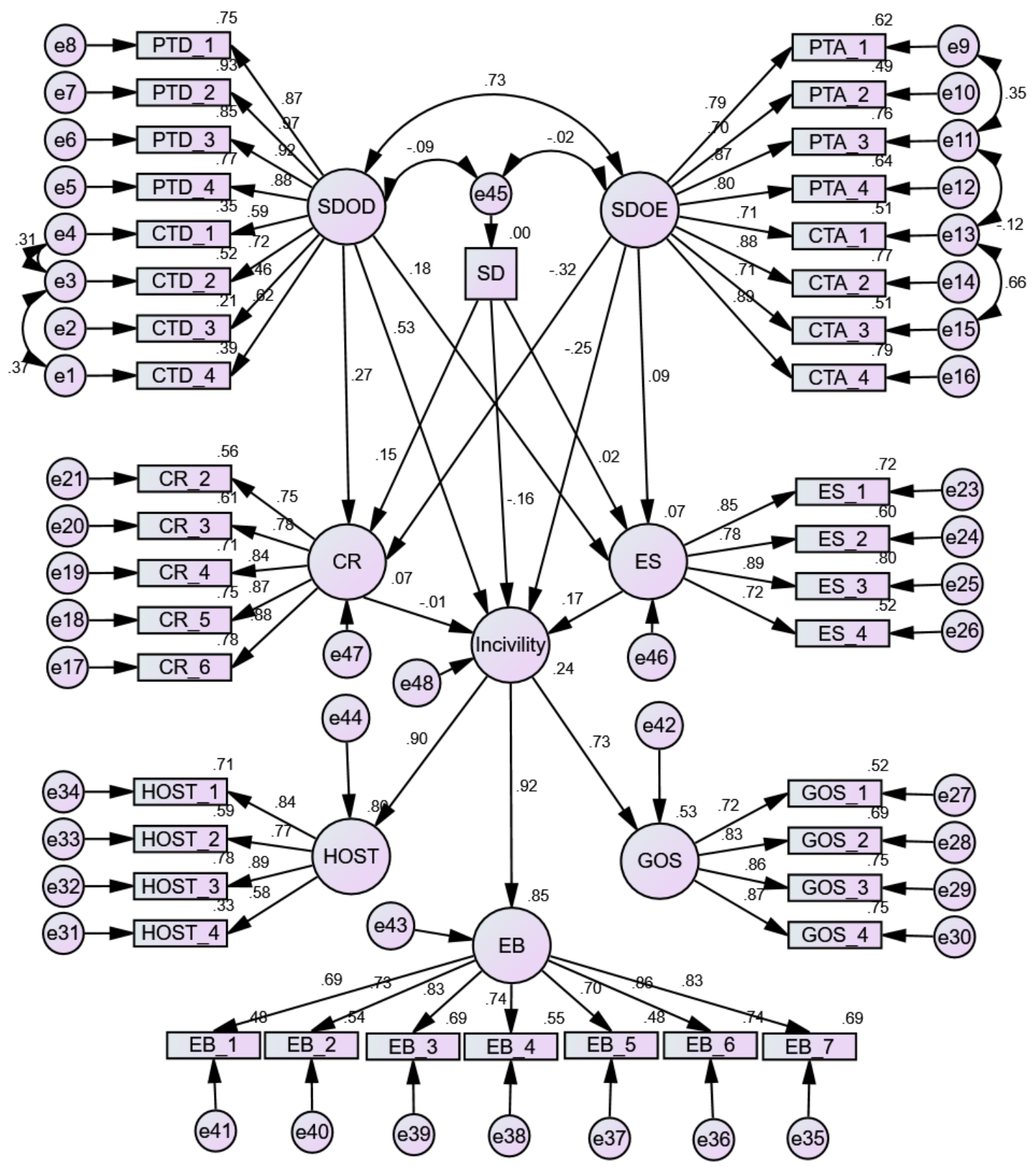

Figure 5. High workgroup civility climate model with standardized coefficients. SDOD = Intergroup Dominance; SDOE = Intergroup Antiegalitarianism; SD = Social Desirability; $\mathrm{CR}=$ Cognitive Reappraisal; ES = Expressive Suppression; Incivility = Instigated Incivility; HOST = Hostility; EB = Exclusionary Behavior; GOS = Gossip. 


\section{Testing Research Hypothesis $\mathrm{H}_{4}$}

The results from the testing of $H_{4}$ are presented next. The effect sizes were evaluated follwing Cohen's (1988) criteria for evaluating effect sizes, thus path coefficients $< \pm .28$ are small effects; medium effects range from $\pm .28-.49$; and, large effects are greater than \pm .49 (Cohen, 1988).

The direct path from SDO-D to instigated incivility was not moderated by workgroup civility climate. There was no significant difference between the low civility climate $(\beta=.50, p<.01)$ and the high civility climate $(\beta=.53, p<.01)$ groups $(z=-0.61$, $\mathrm{p}>.05$ ). Intergroup dominance (SDO-D) is a strong predictor of instigated incivility for both low and high workgroup civility climate groups with a large effect size in each group.

The direct path from SDO-E to instigated incivility was moderated by civility climate. There was a significant difference between the low civility climate $(\beta=-.07, p>.05)$ and the high civility climate $(\beta=-.25, p<.01)$ groups $(z=3.06, \mathrm{p}<.05)$. There was no evidence of a relationship between SDO-E traits and instigated incivility in the low civility climate workgroup. Within the high civility climate workgroup, strong SDO-E traits significantly reduced instigated incivility with a small effect size.

The direct path from SDO-D to expressive suppression was not moderated by civility climate. There was no significant difference between the low civility climate $(\beta=$ $.24, p<.05)$ and the high civility climate $(\beta=.18, p<.05)$ workgroups $(z=0.94, p>$ .05). In both low and high civility climate workgroups, however, strong SDO-D traits predicted the use of the emotion regulation strategy of expressive suppression with a small effect size. 
The direct path from SDO-E to cognitive reappraisal was moderated by civility climate. There was a significant difference between the low civility climate $(\beta=-.10, p$ > $.05)$ and the high civility climate $(\beta=-.31 p<.01)$ workgroups $(z=3.62, \mathrm{p}<.001)$. There was no evidence of a relationship between SDO-E traits and the use of the emotion regulation strategy of cognitive reappraisal in the low civility climate workgroup. Within the high civility climate workgroup, strong SDO-E traits significantly reduced the use of the emotion regulation strategy of cognitive reappraisal with a medium effect size.

The direct path from SDO-E to expressive suppression was moderated by civility climate $(z=-2.90, \mathrm{p}<.01)$. However, in both workgroup civility climate models these paths were not significant. This finding is consistent with the non-significant path between SDO-E and expressive suppression that was found in the mediation model (Figure 3). There is no evidence of a relationship between SDO-E and expressive suppression in both low and high civility climate workgroups. The moderation test was significant due to the opposite direction of the regression weights in the two moderator models. In the low civility climate group, SDO-E had a positive path coefficient, while in the high civility climate group the path coefficient was negative.

The overall indirect paths of SDO-D and SDO-E to instigated incivility through expressive suppression and cognitive reappraisal were not moderated by civility climate. However, in the low civility climate SEM, the overall indirect effect of SDO-D on instigated incivility through the mediators expressive suppression and cognitive reappraisal was significant $(\beta=.03, p<.05)$. The results of the specific indirect effects showed evidence for a significant indirect effect for SDO-D on instigated incivility most likely through the emotion regulation strategy of expressive suppression $(\beta=.01, p<$ 
$.05)$. There is evidence that in workgroups with a low workgroup civility climate, expressive suppression may indirectly reduce the large direct effect of strong SDO-D traits on instigated incivility. This result is consistent with significant specific indirect effect of SDO-D on incivility through expressive suppression that was tested in the mediation SEM. Path differences in effect are expressed visually in Figure 6 for ease of interpretation. Table 9 presents the results of the moderation tests of workgroup civility climate. Table 10 provides the results of the specific indirect effects for the low workgroup civility climate SEM.

As there were differences in paths between the low civility climate and high civility climate workgroups, $\mathrm{H}_{4}$ was supported.

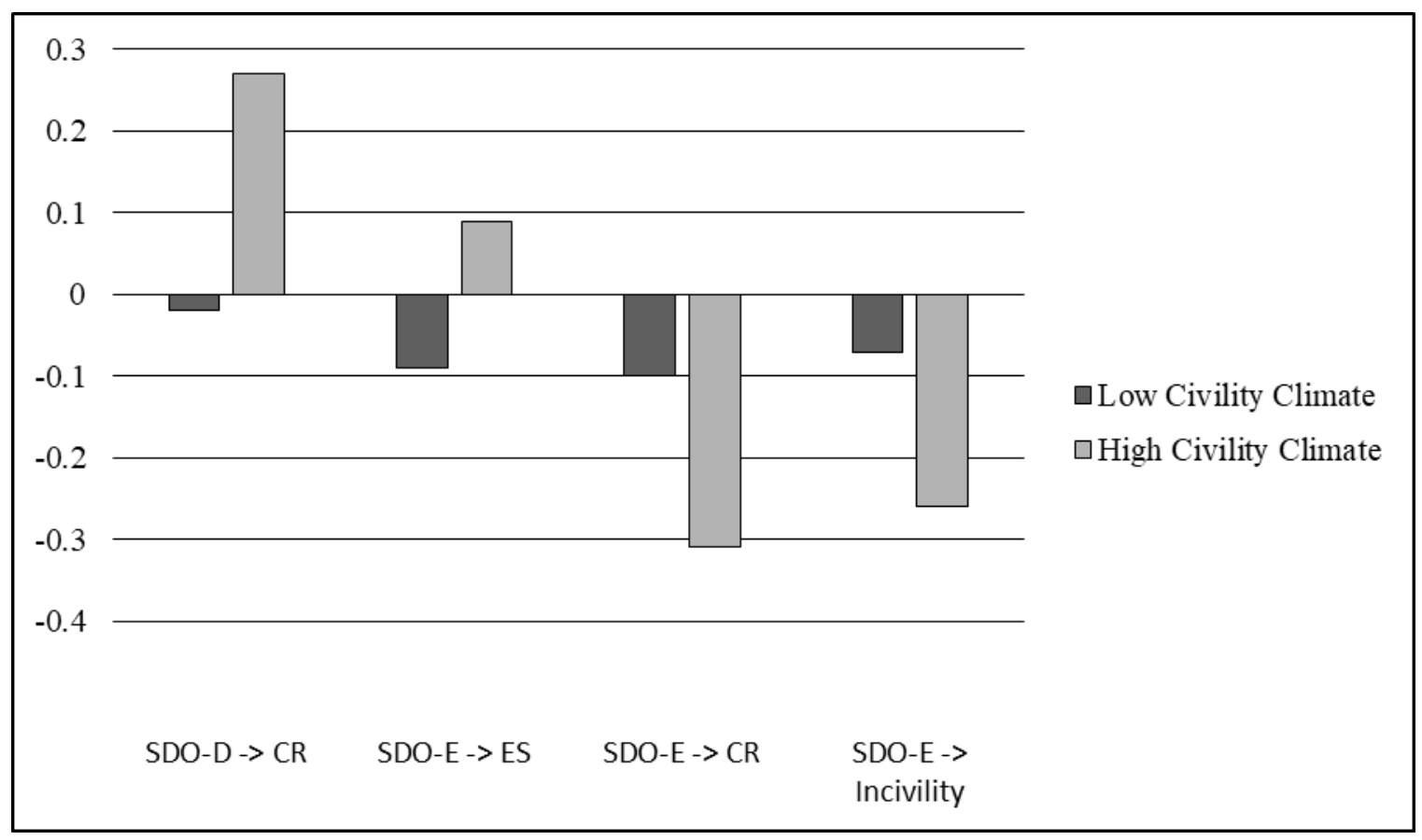

Figure 6. Significant path differences between moderator groups. SDO-D = Intergroup Dominance; SDO-E = Intergroup Antiegalitarianism; CR = Cognitive Reappraisal; ES = Expressive Suppression; Incivility = Instigated Incivility. 
Table 9

Moderation of Workgroup Civility Climate on Path Strengths

\begin{tabular}{|c|c|c|c|c|}
\hline Path & $\Delta z$ score & $p$ & Low group $\beta$ & $\begin{array}{c}\text { High group } \\
\beta\end{array}$ \\
\hline $\mathrm{CR} \leftarrow \mathrm{SDO}-\mathrm{D}$ & -4.66 & $* * * .000$ & -.02 & .27 \\
\hline $\mathrm{ES} \leftarrow \mathrm{SDO}-\mathrm{E}$ & -2.90 & $* * .006$ & -.09 & .09 \\
\hline $\mathrm{CR} \leftarrow \mathrm{SDO}-\mathrm{E}$ & 3.62 & $* * .001$ & -.10 & -.32 \\
\hline $\mathrm{ES} \leftarrow \mathrm{SDO}-\mathrm{D}$ & 0.94 & .257 & .24 & .18 \\
\hline $\mathrm{CR} \leftarrow \mathrm{SD}$ & -0.62 & .330 & .12 & .15 \\
\hline $\mathrm{ES} \leftarrow \mathrm{SD}$ & 0.35 & .375 & .04 & .02 \\
\hline Incivility $<\mathrm{ES}$ & -0.70 & .312 & .12 & .17 \\
\hline Incivility $\leftarrow \mathrm{CR}$ & -0.70 & .311 & -.05 & -.01 \\
\hline Incivility $\leftarrow$ SDO-E & 3.06 & $* * .004$ & -.07 & -.25 \\
\hline Incivility $\leftarrow$ SDO-D & -0.61 & .331 & .50 & .53 \\
\hline Incivility $\leftarrow$ SD & 0.68 & .316 & -.11 & -.16 \\
\hline \multicolumn{5}{|l|}{ Indirect Effects } \\
\hline Incivility $\leftarrow$ SDO-E & -0.40 & .368 & -.01 & .02 \\
\hline Incivility $\leftarrow$ SDO-D & 0.05 & .398 & .03 & .03 \\
\hline
\end{tabular}

Note. Low Civility $(N=503)$. High Civility $(N=524)$. SDO-D = Intergroup Dominance; SDO-E = Intergroup Antiegalitarianism; CR = Cognitive Reappraisal; ES = Expressive Suppression; Incivility = Instigated Incivility; SD = Social Desirability. $\leftarrow=$ Path. $* p<.05 . * * p<.01 . * * * p<.001$.

Table 10

Specific Indirect Effects for Low Workgroup Civility Climate Model

\begin{tabular}{lcccc}
\hline & & \multicolumn{3}{c}{$95 \%$ Confidence interval } \\
\cline { 3 - 4 } Path & Estimate & Lower & Upper & $p$ \\
\hline & & & & \\
SDOE on Incivility through CR & .00 & .00 & .01 & .255 \\
SDOE on Incivility through ES & .00 & -.02 & .00 & .264 \\
SDOD on Incivility through CR & .00 & .00 & .01 & .548 \\
SDOD on Incivility through ES & .01 & .00 & .04 & $* .012$ \\
\hline
\end{tabular}

Note. SDO-D = Intergroup Dominance; SDO-E = Intergroup Antiegalitarianism; CR = Cognitive Reappraisal; ES = Expressive Suppression; Incivility = Instigated Incivility. $* p<.05$. 


\section{Summary of the Results}

The findings through SEM analyses showed support for $H_{l a}$, with a positive effect between intergroup dominance (SDO-D) and instigated incivility, which was not moderated by workgroup civility climate. Hypothesis $l_{b}$ was not supported, the path from intergroup antiegalitarianism (SDO-E) to instigated incivility was significant, but negative; $H_{1 b}$ was moderated by workgroup civility climate. Hypothesis $2_{a}$ was partially supported, there was a positive effect between SDO-D and the emotion regulation strategy of expressive suppression, which was not moderated by workplace civility climate. There was no evidence of a relationship between SDO-D and emotion regulation strategy cognitive reappraisal in the mediation SEM, but the path was moderated by workgroup civility climate. Hypothesis $2 b$ was partially supported, there was a negative effect between SDO-E and the emotion regulation strategy of cognitive reappraisal, which was moderated by workgroup civility climate. There was no evidence of a relationship between SDO-E and the emotion regulation strategy of expressive suppression in the mediation SEM, but the path was moderated by workgroup civility climate. There was no evidence of an overall indirect effect between both predictor variables (SDO-D and SDO-E) on the outcome of instigated incivility, thus $H_{3 a}$ to $H_{3 d}$ were not supported, these paths were not moderated by workgroup civility climate. Further testing for specific indirect effects indicated a positive indirect effect for SDO-D on instigated incivility through the emotion regulation strategy of expressive suppression. In contrast, the specific indirect effect for SDO-E on instigated incivility was positive and most likely attributed to the emotion regulation strategy of cognitive reappraisal. The specific indirect effects will be interpreted with caution due to the potential of a Type I 
error. In the low workgroup civility climate SEM, there was a significant overall indirect effect for SDO-D and instigated incivility through both emotion regulation strategies (cognitive reappraisal and expressive suppression). The results of further testing for specific indirect effects indicated that in the low civility climate group, there was evidence of a significant indirect effect for SDO-D on instigated incivility though the emotion regulation strategy of expressive suppression.

Figure 5 presents a visual of the mediation model with the paths moderated by workgroup civility climate. Moderated paths are represented by the dashed lines. A discussion of the results of this analysis is presented in the following chapter.

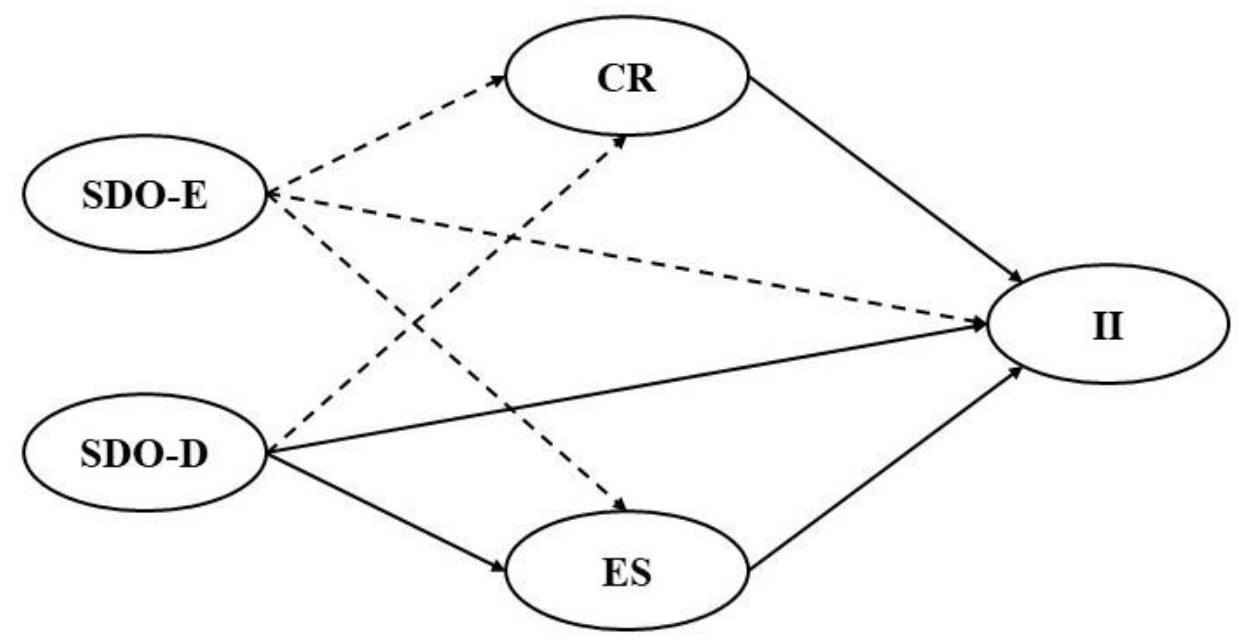

Figure 7. Mediation model with moderated paths. Note. Dashed lines indicate the path is moderated by workgroup civility climate. SDO-D = Intergroup Dominance; SDO-E = Intergroup Antiegalitarianism; CR = Cognitive Reappraisal; ES = Expressive Suppression; II = Instigated Incivility. 


\section{CHAPTER V \\ DISCUSSION}

Chapter V provides a brief summary of the study, followed by a discussion of the results. Implications for theory, practice, and limitations and recommendations for future research are offered followed by a conclusion of the chapter.

\section{Summary of the Study}

The negative individual, workgroup, and organizational outcomes of uncivil workplace behavior are well documented by incivility scholars (Cortina et al., 2017; Reio \& Ghosh, 2009; Schilpzand et al., 2016). Workplace incivility is defined as "low intensity deviant behavior with ambiguous intent to harm the target, [that is] in violation of workplace norms for mutual respect" (Andersson \& Pearson, 1999, p. 457). When social norms proscribe the expression of overt discrimination, a prejudiced individual may turn to incivility as a more socially acceptable way to exert their power over others (Githens, 2011). Prejudice-driven negative workplace behavior is gaining the attention of organizational scholars who have linked individual levels of social dominance orientation (Aiello et al., 2018; Daniels et al., 2015; Hu \& Liu, 2017; Shao et al., 2011) with workplace behaviors that often bypass formal sexual harassment and discrimination laws (Binggeli et al., 2014; Cortina, 2008).

In response to the need to create productive, healthy, respectful, and socially responsible workplace environments, there is an urgent call for HRD researchers and practitioners to develop and support strategies that reduce the incidence and impact of uncivil workplace behavior (Brunetta \& Reio, 2016; Byrd, 2018; Callahan, 2011; Estes \& Wang, 2008; Githens, 2011; Mizzi \& Rocco, 2013; Reio \& Sanders-Reio, 2011; Trudel 
$\&$ Reio, 2011). However, there is a significant shortage of research on instigators of incivility and on variables that may address how, why, or when workplace incivility occurs (Cortina et al., 2018; Schilpzand et al., 2016). The proposed model of instigated incivility tested in this research suggested that employees with strong social dominance traits are more likely to instigate uncivil workplace behavior (Daniels et al., 2015; Hu \& Liu, 2017; Shao et al., 2011). The model also explored how or why instigated incivility unfolds within organizations and suggested that uncivil behavior may be explained by the emotion regulation strategies of cognitive reappraisal and expressive suppression (Grandey, 2000; Grandey \& Gabriel, 2015; Van Hiel et al., 2006), and that norms of workgroup civility climate may specify when instigated incivility occurs (Cortina et al., 2018; Walsh et al., 2012; 2018).

The purpose of the study was to develop and test a parallel mediator model of instigated incivility moderated by workgroup civility climate norms. The research examined the influence of the mediation of the emotion regulation strategies of cognitive reappraisal and expressive suppression, on two types of social dominance orientation support for intergroup dominance (SDO-D) and intergroup antiegalitarianism (SDO-E) on the outcome of instigated incivility. Two research questions guided the study: Do the emotion regulation strategies of cognitive reappraisal and expressive suppression mediate the relationship between the two types of social dominance orientation (SDO-D and SDO-E), and what are the moderating effects of workgroup civility climate on the proposed model of instigated incivility? The model tested four major research hypotheses ( $H_{l a}$ to $H_{3 d}$, and $H_{4}$ ) to examine these questions: 
Hypothesis 1a: There is a statistically significant positive relationship between SDO-D and instigated incivility.

Hypothesis ${ }_{1 b}$ : There is a statistically significant positive relationship between SDO-E and instigated incivility.

Hypothesis $2 a$ : There is a statistically significant relationship between SDO-D and the mediators expressive suppression and cognitive reappraisal.

Hypothesis ${ }_{2 b}$ : There is a statistically significant relationship between SDO-E and the mediators expressive suppression and cognitive reappraisal.

Hypothesis 3a: Expressive suppression mediates the relationship between SDO-D and instigated incivility, while controlling for SDO-E.

Hypothesis ${ }_{3 b}$ : Cognitive reappraisal mediates the relationship between SDO-D and instigated incivility, while controlling for SDO-E.

Hypothesis ${ }_{3 c}$ : Expressive suppression mediates the relationship between SDO-E and instigated incivility, while controlling for SDO-E.

Hypothesis 3d: Cognitive reappraisal mediates the relationship between SDO-E and instigated incivility, while controlling for SDO-D.

Hypothesis 4: The paths of the model are moderated by workgroup civility climate such that the strength or direction of one or more paths are significantly different for subsamples from different civility climates.

An Internet-based survey was used to investigate the relation among the variables of interest. A population of MTurk workers provided the sample. Structural equation modeling (SEM) with mediation and moderation analyses were used to test the hypothesized model of instigated incivility and examine the research hypotheses. 


\section{Discussion of the Results}

The following discussion of the results is guided by theory and research and will follow the research hypotheses $H_{l a}$ to $H_{3 d}$. The results of the moderation analyses $\left(H_{4}\right)$ are discussed in conjunction with $H_{1 a}$ to $H_{3 d}$ as each of these hypotheses were tested for the moderating effects of workgroup civility climate. As recommended by Podsakoff et al. (2013) and Reio (2010), the possible effect of social desirability was statistically controlled when testing the theoretical models. What this means is that the effects (path coefficients) demonstrated in the models were free of the possible confounding influence of social desirability. This represents a contribution to the research literature in that social desirability has not been controlled in most incivility studies.

\section{Hypothesis 1a}

Hypothesis $l_{a}$ posited a relationship between the social dominance orientation dimension of SDO-D and instigated incivility. Results from the SEM mediation analysis indicated a significant and positive relationship from SDO-D to instigated incivility, supporting $H_{1 a}$. Employees who had strong SDO-D traits were more likely to instigate incivility toward a coworker. This finding supports Cortina's (2002) suggestion of a possible link between social dominance, as proposed by social dominance theory (Sidanius \& Pratto, 1993), and workplace incivility. Social dominators have the predisposition to engage in many forms of uncivil behavior to enhance their relative position by devaluing others (Kupper et al., 2010). Employees who foster negative attitudes toward coworkers may experience negative emotions such as hostility or emotional conflict (Jehn, 1999; Kim et al., 2012). Intergroup dominance (SDO-D) involves a preference for overt and aggressive behaviors (Ho et al., 2012), observed in 
the positive and large effect size of the path from SDO-D to instigated incivility in this study. This finding is consistent with previous research on the positive relationship between intergroup dominance and instigated incivility (Daniels et al., 2015).

Organizational scholars have linked SDO-D also to other forms of negative interpersonal behaviors, such as interpersonal deviance (Shao et al., 2011), abusive supervision (Hu \& Liu, 2017; Shao et al., 2011), and the use of harsh power tactics (Aiello et al., 2018).

The moderation analysis results of workgroup civility climate on the direct path from SDO-D to instigated incivility revealed an important finding. Workgroup norms of civility climate did not moderate the relationship between SDO-D and instigated incivility. There was no significant difference between civil and uncivil workgroup civility climate groups on the strength of the relationship between SDO-D and instigated incivility. In both workgroup civility climate groups, employees who had strong SDO-D traits had a significant and positive relationship with instigated incivility, with a large effect size in both moderator groups. This finding contrasts with previous research which indicates that a generalized workplace climate of respect reduces workplace incivility (Andersson \& Pearson, 2005; Foulk et al., 2016; Glomb \& Liao, 2013; Holm et al., 2015; Walsh et al., 2012, 2018). This line of inquiry suggests that uncivil workplace behavior should occur with less frequency within organizations that support norms for civility that encourage employees to behave in a respectful manner (Gill \& Sypher, 2009; Leiter et al., 2012; Walsh et al., 2012). Yet, there are other streams of incivility research which are in conceptual congruence with the findings of this study. The finding of this study supports the research by Miner and colleagues (2018) which concludes that individual assessments of uncivil behavior may be linked to larger social forces external to the work 
environment which guide behavioral expectations. Similarly, scholars have linked SDOD traits with extreme behaviors such as physical violence (Ho et al., 2015), overt racism (Ho et al., 2012), blatant dehumanization (Kiteily et al., 2015), and hostile sexism (Bergh et al., 2015). This suggests that the link between SDO-D and extreme behaviors may impact how socially dominant employees perceive the less extreme, more mundane behaviors referenced in the workgroup civility climate questionnaire. Daniels and colleagues (2015) found a significant correlation between social dominance and psychological entitlement, which may further explain the ineffectiveness of workgroup norms of civility climate on employees with strong SDO-D traits.

\section{Hypothesis 1b}

Hypothesis $l_{b}$ posited a relationship between the social dominance orientation dimension of SDO-E (intergroup antiegalitarianism) and instigated incivility. Results from the SEM mediation analysis indicated there was a significant and negative path from SDO-E to instigated incivility. Employees who had strong SDO-E traits were less likely to instigate incivility. This finding was surprising because SDO-E is a dimension of social dominance orientation treated by researchers as a milder, yet consistent manifestation of social dominance (Ho et al., 2012; 2015). This researcher expected a positive, but smaller effect for the relationship between SDO-E and instigated incivility. While SDO-D is designed to measure the original signifiers of SDO, as developed by Sidanius and Pratto (1993) - a disposition to support group-based dominance with a preference for overt and aggressive behaviors - SDO-E addresses the disposition to oppose group-based equality (Ho et al., 2012), and is characterized by subtle and covert forms of prejudice (Bergh et al., 2015; Hindriks et al., 2014; Ho et al., 2012, 2015; 
Kiteily et al., 2015). This result supports previous research which indicates the two related dimensions of SDO can indeed predict different outcomes (Ho et al., 2012, 2015; Jost \& Thompson, 2000; Kugler et al., 2010).

Scholars have linked SDO-E with political conservatism (Bergh et al., 2015; Ho et al., 2012, 2015). Ho and colleagues (2015) suggested that individuals high in SDO-E may be more politically sophisticated compared to individuals high in SDO-D and conclude that SDO-E represents a less severe approach to achieve and maintain inequality. For example, Kiteily at al. (2015) found that SDO-D predicted blatant dehumanization, while SDO-E showed no evidence of a relationship with blatant dehumanization. Bergh et al. (2015) found a strong negative relationship between the personality trait of honesty-humility and SDO-D, while it was unrelated to SDO-E. Ho and colleagues (2015) reported a strong relationship between SDO-D and Machiavellianism, narcissism, and psychopathy, while SDO-E showed weaker relationships with these constructs.

Results from the moderation analysis also indicated that workgroup civility climate norms moderated the relationship of SDO-E and instigated incivility. Intergroup antiegalitarianism (SDO-E) had a significant and negative relationship with instigated incivility in the high workgroup climate group. There was no evidence of a relationship between SDO-E and instigated incivility in the low workgroup civility climate group. This finding highlights the important influence of workplace climate on individuals with "milder" dominance traits and is consistent with previous research which suggests that a generalized workplace climate of respect can reduce workplace incivility (Andersson \& 
Pearson, 2005; Foulk et al., 2016; Glomb \& Liao, 2013; Holm et al., 2015; Walsh et al., 2012, 2018).

\section{Hypothesis 2a}

Hypothesis 2 a posited a relationship between SDO-D and the emotion regulation strategies of cognitive reappraisal and expressive suppression. As discussed in a previous chapter, emotion regulation (Gross, 1998b) refers to the actual strategies employees use to perform the emotion work required to accommodate the feeling rules established by the organization. The expressive suppression of negative emotions is a commonly used emotion regulation strategy in social life (Gross et al., 2006), and scholars have linked expressive suppression to negative social consequences, in and outside the work environment (e.g., Butler et al., 2003, 2014).

Results from the mediation analysis showed no evidence of a relationship between SDO-D and cognitive reappraisal. However, there was evidence of a significant and positive relationship between SDO-D and expressive suppression. These findings partially support $H_{2 a}$. Increasing SDO-D scores predicted a higher use of the emotion regulation strategy of expressive suppression. This finding is consistent with previous research which indicates that social dominators tend to withhold emotional expressions (Van Hiel et al., 2006). Kim et al. (2013) theorized that in-group favoritism and outgroup discrimination increases the use of response-focused emotion regulation strategies, such as expressive suppression. Response-focused strategies occur when an emotion is already underway. That is, after the generation of response tendencies (Gross, 1998b; Hochschild, 1983). Because expressive suppression of emotion influences the modulation of emotion response tendencies once they are triggered, suppression is expected to have 
generally more negative implications for affective and social functioning (Gross, 1998b;

Gross \& Levenson, 1993; Hochschild, 1983). Individuals engaging in emotion suppression are putting on a mask by suppressing or faking emotions (Hochschild, 1983; Gabriel et al., 2015).

In the model tested, workgroup civility climate did not moderate the relationship between SDO-D and the emotion regulation strategy of expressive suppression. In both workgroup civility climate groups, increasing SDO-D scores predicted higher use of the emotion regulation strategy of expressive suppression with no significant difference between the two groups.

The result of the moderation analysis indicated a significant difference in the paths from SDO-D to cognitive reappraisal between the low and high workgroup civility climate groups. In workplace environments with high levels of workgroup civility climate, employees who had strong SDO-D traits had a significant and positive relationship with the emotion regulation strategy of cognitive reappraisal. In contrast, there was no evidence of a relationship between SDO-D and cognitive reappraisal in the low workgroup civility climate group. This result signals the influence of workplace civility norms for the use of more adaptive emotion regulation strategies. Cognitive reappraisal is an antecedent-focused emotion regulation strategy which occurs before emotion response tendencies have activated and have changed behavior and physiological responses (Gross, 1998b; Hochschild, 1983). Greater use of cognitive reappraisal is associated with positive outcomes such as greater psychological adjustment and improved interpersonal functioning (Gross, 2002; John \& Gross, 2004, 2007). The results of the moderation analysis indicated that high workgroup civility climates activated the 
use of the emotion regulation strategy of cognitive reappraisal among employees with strong SDO-D traits. This finding is consistent with Ekman's (1973) theory that organizational display rules are behavioral standards established by the organization to indicate which emotional expressions are considered acceptable or not acceptable. Individuals use cognitive reappraisal in the attempt to modify feelings to match the required display rules of the organization (Gabriel et al., 2015).

\section{Hypothesis 2b}

Hypothesis $2 b$ posited a relationship between SDO-E and the emotion regulation strategies of cognitive reappraisal and expressive suppression. Results from the mediation analysis indicated a significant, but negative, relationship between SDO-E and cognitive reappraisal. Increasing SDO-E scores predicted lower use of the emotion regulation strategy of cognitive reappraisal. Workgroup civility climate moderated the direct path of SDO-E and the emotion regulation strategy of cognitive reappraisal. In the low workgroup civility climate there was no evidence of a relationship between SDO-E and cognitive reappraisal. In contrast, employees with strong SDO-E scores in the high workgroup civility climate group showed a significant decrease in the use of the emotion regulation strategy of cognitive reappraisal. There was no evidence of a relationship between SDO-E and expressive suppression; $H_{2 b}$ was partially supported.

The direct path from SDO-E to the emotion regulation strategy of expressive suppression was not significant in both low and high workgroup civility climate structural models. However, the moderation analysis result indicated a significant difference on the direction of the path coefficients between the two workgroup civility climate groups. In low workgroup civility climates, the regression coefficient between SDO-E and 
expressive suppression was negative. In contrast, the regression coefficient in the high workgroup civility climate group was positive.

The results of the mediation and moderation analyses for $H_{2 b}$ did not provide evidence about which emotion regulation strategy employees with strong SDO-E traits use, but indicated only which emotion regulation they did not use. Intergroup antiegalitarianism (SDO-E) traits significantly decreased the use of cognitive reappraisal and were not related to expressive suppression in the mediation SEM. The moderation analysis results indicated significant differences between workgroup civility climate groups on the SDO-E to cognitive reappraisal paths. There was a significant decrease in the use of cognitive reappraisal in the high workgroup civility climate group. Cognitive reappraisal and expressive suppression are the most frequently used emotion regulation strategies in everyday life (Gross, 2014; Gross \& John, 2003; Hochschild, 1983). There is a possibility that individuals with SDO-E traits regulate emotions with strategies that were not measured in this study.

\section{Hypotheses 3a to 3d}

Hypotheses $3_{a}$ to $3_{d}$ posited specific indirect effects from SDO-D and SDO-E to instigated incivility through the emotion regulation strategies of expressive suppression and cognitive reappraisal. The sum of the specific indirect effects for SDO-D and SDO-E through both the emotion regulation strategies (cognitive reappraisal and expressive suppression) on instigated incivility showed no evidence of an overall indirect effect for both dimensions of social dominance. This finding indicated that the combined effects of cognitive reappraisal and expressive suppression did not produce evidence to explain how or why the direct relationship between both social dominance predictors and instigated 
incivility existed. There was no evidence to support the moderation of workgroup civility climate on the overall indirect effects for SDO-D and SDO-E on instigated incivility through the emotion regulation strategies of expressive suppression and cognitive reappraisal.

This researcher further tested for specific indirect effects to better understand the inner workings of the model despite the potential for a Type I error (Macho \& Lederman, 2009). The results of the measurements of the specific indirect effects in the mediation SEM indicated a likely positive indirect effect for SDO-D on instigated incivility through the emotion regulation strategy of expressive suppression $\left(\mathrm{H}_{3 a}\right)$. This finding is consistent with emotion regulation theory (Gross \& John, 2003) and previous research supporting the relationship between social dominance and expressive suppression (Kim et al., 2013; Van Hiel et al., 2006). The evidence from this study indicated that among employees who had strong SDO-D traits, the direct relationship with instigated incivility was significantly reduced by the indirect effect of the emotion regulation strategy of expressive suppression.

An additional finding from the analysis of the low workgroup civility climate SEM indicated some evidence of an indirect effect from SDO-D to instigated incivility through the emotion regulation strategy of expressive suppression. This result is consistent with significant specific indirect effect of SDO-D on instigated incivility through expressive suppression that was tested in the mediation SEM. Individuals who employ the emotion regulation strategy of expressive suppression are disinclined to share their emotions and may actively avoid interactions that give rise to strong emotions (Gross \& John, 2003). The results of this study indicated that in the low workgroup 
civility climate group, strong SDO-D traits reduced instigated incivility through the indirect effect of expressive suppression. These findings suggest expressive suppression may be useful for reducing instigated workplace incivility in general, and specifically in workplaces with uncivil workgroup climate norms.

The results of this study are consistent with the link between prejudice and increased response-focused emotion regulation strategies such as expressive suppression (Kim et al., 2013; Van Hiel et al., 2006), and in contrast with previous research suggesting that expressive suppression is expected to have generally more negative implications for social functioning (Gross, 1998b; Gross \& Levenson, 1993; Gross \& Thompson, 2007; Hochschild, 1983).

Finally, the results from the mediation SEM analysis revealed additional important findings for a study on instigated incivility. First, increasing expressive suppression scores predicted increasing instigated incivility scores. In contrast, increasing cognitive reappraisal scores predicted decreasing instigated incivility scores. This finding suggests that the emotion regulation strategies of cognitive reappraisal and expressive suppression have an opposite effect on instigated incivility. Expressive suppression increased instigated incivility, while cognitive reappraisal reduced instigated incivility. Greater use of the emotion regulation strategy of cognitive reappraisal is empirically linked to adaptive emotional and social outcomes (Denny \& Ochsner, 2014; Gross \& John, 2003; Troy et al., 2010).

\section{Implications for Theory}

Andersson and Pearson (1999) emphasize that workplace incivility is characterized by an ambiguous intent to harm the recipient of the uncivil behavior. 
Miner and colleagues (2017) identified a series of assumptions that have limited the advancement of workplace incivility theory. One of these assumptions is that workplace incivility is theorized as having an ambiguous intent to harm the target of uncivil behavior. Cortina (2008) opened a door to challenge this assumption with selective incivility theory, suggesting that instigated incivility may be an outlet for racist and sexist individuals to intentionally harm their targets. The results of this study further challenge the assumption that incivility may be ambiguous in its intent to harm the target. This research extends social dominance theory to instigated incivility, thus extending the theoretical lens offered by Cortina (2008) that workplace incivility may include the intent to harm selective targets. The instigated incivility model proposed in this study links intergroup dominance (SDO-D) with instigated incivility, which numerous scholars have empirically linked to prejudice and discrimination (Bergh et al., 2015; Ho et al., 2012; Ho et al., 2015; Hindriks et al., 2014; Kiteily et al., 2015; Kteily et al., 2011; Kupper et al., 2010; Pratto et al., 1994; Sibley \& Duckitt, 2008).

The present model of instigated incivility contextualizes the role of emotion regulation in the instigated incivility literature. Findings from this study have significant theoretical implications which may guide our understanding of how instigated uncivil behavior unfolds in the workplace, rather than how emotions are regulated in the context of experienced incivility. There is a gap in the workplace incivility literature addressing the role of emotion regulation on instances of instigated incivility. The results of this study are consistent with the emotion regulation framework, which suggests that the way we regulate our emotions results in different cognitive and behavioral outcomes (Angie et 
al., 2011; Hulsheger \& Schewe, 2011; Kammeyer-Mueller et al., 2013; Lench et al., 2011; Mesmer-Magnus et al., 2012).

In this study, the emotion regulation strategy of cognitive reappraisal was negatively related to incivility, while the emotion regulation strategy of expressive suppression was positively related to instigated incivility. An understanding of the role that specific emotion regulation strategies such as cognitive reappraisal and expressive suppression play on shaping behavioral outcomes opens a theoretical lens for further investigation of instigated incivility. The most recent and comprehensive review of the incivility literature called upon the need to expand research on mediators and moderators of uncivil workplace behavior to advance workplace incivility theory (Schilpzand et al., 2016). This study offers cognitive reappraisal and expressive suppression as variables for theorizing how individual perceptions of others influence individual and organizational outcomes based on emotion regulation preferences.

Recent developments in social dominance theory indicate social dominance orientation consists of two related, but distinct constructs that predict different behavioral outcomes - SDO-D and SDO-E (Ho et al., 2012, 2015). The current study confirmed the distinct predictive ability of the two social dominance orientation dimensions on the outcome of instigated incivility. This finding supports current social dominance theory that two forms of hierarchy-enhancing ideology may indeed result in different behavioral outcomes. Intergroup dominance (SDO-D) was positively related to instigated incivility, while intergroup antiegalitarianism (SDO-E) was negatively related to instigated incivility. Although the results from this study are consistent with previous research conducted by scholars who found that the two SDO dimensions predicted different 
outcomes (Bergh et al., 2015; Hindricks et al., 2015; Ho et al., 2012, 2015; Kiteily et al., 2015), there is a need to further test the two dimensions of SDO to advance social dominance theory.

\section{Implications for HRD Practice}

A radical perspective of HRD includes the goal of securing the respectful treatment of all employees within their workplaces, and to take action against any system that seeks to impose or maintain contrary views (Byrd, 2018). The results of this study are correlational and need to be further assessed by experimental research. However, based on the nature and the results of this study, this researcher suggests that a radical perspective may be necessary to best apply the findings in the field of HRD.

The results indicated that high levels of SDO-D predict instigated workplace incivility. Human resource practitioners can use predictors of workplace incivility to identify employees who are more likely to instigate uncivil behaviors (Reio \& Ghosh, 2009). Human resource departments and HRD professionals have the opportunity to include an assessment of SDO-D within the hiring procedures of their organizations to help identify likely instigators. Intergroup dominance is not only linked to instigated incivility, as found in this study, but is also a predictor of other forms of deviant, counterproductive, or unethical workplace behaviors (Aiello et al., 2018; Hu \& Liu, 2017; Shao et al., 2011). Organizations that wish to respect anti-discrimination and harassment laws may need to consider the implications of hiring employees with strong SDO-D traits.

The results of this study also emphasize the importance of creating a workplace culture with high normative expectations of civil behavior. Organizations need to 
establish very clear behavioral expectations that are not vague in language and open to subjective interpretation. Policies that define workplace incivility need to be explicit in their proscription of behaviors that are considered uncivil. A starting place for HRD practitioners is to use existing incivility measures (see Blau \& Andersson, 2005; Cortina et al., 2001; Gray et al., 2016) to specify the type of behaviors employees should not deploy in the workplace. Once organizations produce explicit policies of workplace incivility, these specific expectations need to be included as part of the performance appraisal process. Performance appraisals can play an important role in the establishment and reinforcement of organizational civility expectations (Kunkel \& Davidson, 2014).

The results of this study indicated that the both types of social dominance orientations and instigated incivility had a negative relationship with the emotion regulation strategy of cognitive reappraisal. Denny and Ochsner (2014) found that longitudinal trainability of cognitive reappraisal is linked to improved ability to downregulate negative emotions that may otherwise lead to unwanted behavioral manifestations. HRD practitioners are positioned to develop the educational agendas and diversity initiatives of organizations to promote a climate of respect in employee relations (Mizzi \& Rocco, 2013). Human resource professionals can implement emotion regulation trainings to encourage the use of cognitive reappraisal to regulate negative emotions. Emotion regulation is known to be a critical skill for managing interpersonal conflict (Angie, Connelly, Waples, \& Kligyte, 2011; Hülsheger \& Schewe, 2011; KammeyerMueller et al., 2013; Lench et al., 2011; Mesmer-Magnus et al., 2012).

Unfortunately, there is a lack of empirically sound practical interventions for the problem of workplace incivility (Cortina et al., 2017). An existing civility initiative 
documented in the literature as an effective method for lowering incivility and improving civility perceptions is the Civility, Respect, and Engagement in the Workplace (CREW) intervention (Leiter et al., 2011; Osatuke et al., 2009; Spence Laschinger et al., 2012). The intervention was designed to promote positive working relationships and is focused on developing respectful forms of communication (Osatuke et al., 2009). Another documented method for preventing and reducing uncivil workplace behavior is the Toxic Organization Change System (TOCS); the intervention model operates on three levels of the workplace environment (a) organizational, (b) team, and (c) individual (Kusy \& Halloway, 2009). HRD practitioners may want to consider applying these two intervention models to their respective workplaces for the purpose of reducing the likelihood of instigated incivility, especially for those who score high in the SDO-D trait.

\section{Limitations and Recommendations for Future HRD Research}

First, the cross-sectional nature of the present study limits its ability to establish causality between the two dimensions of social dominance orientation, emotion regulation strategies, and instigated incivility. The scope of this study was to identify "causal" paths to be explored by future experimental or longitudinal research methodology. While social scientific research utilizing the MTurk platform is expanding and gaining acceptance, there are certain limitations to its effectiveness. The non-random nature of the sample limits its generalizability as well as perhaps rendering it unrepresentative of specific types of populations (Callegaro et al., 2014). Random sampling would also address the issue of non-response bias. The response rate of the survey was not possible to calculate in this study as the data were collected via Amazon Mechanical Turk (MTurk). All eligible MTurk workers received access to the survey, but 
the number of MTurk workers who received the invitation to participate in the study and chose not to respond was unavailable.

As mentioned in the delimitations, the researcher chose to study "workers" as an operational concept. This research is not studying a specific worksite. It is well known that a variety of factors influence the dynamics of the workplace (e.g., physical environment, work culture, type of workforce). This study did not take into consideration the variety of worksite factors that might have an impact on an individual's behavior. The focus of this research was on how socially dominant individuals regulate emotions in relation to socially established workgroup norms of civility. While it may be possible to generalize the findings to the social category of "workers," the researcher is not able to speak about workers in specific industries (e.g., service sector, industrial sector) with confidence.

The instigated incivility model proposed by this study should be tested then on random samples drawn from populations of full-time workers in specific industries. It is important to extend this study to specific industries to provide a deeper understanding of how and when social dominance orientation operates as a predictor of instigated incivility. Researchers should also consider testing the model in international settings with different ethnic and cultural compositions. The sample in this study consisted of predominantly White American employees and is thus limited for our understanding of how the model operates among different groups. Theoretically, social dominance theory maintains that social dominance operates in all societies (Sidanius \& Pratto, 1999).

This study included workgroup civility climate norms as a contextual moderator of the social dominance and incivility relationship. Workgroup civility climate is 
considered a meso-level factor that measures the environment at the group level of analysis (Miner et al., 2018). Future studies should consider macro-level factors that measure the environment at the industry level of analysis (Miner et al., 2018). Contextual factors may help researchers identify forms of incivility that may be found only in specific contexts, for example, male versus female dominated industries (Cortina et al., 2017; Miner et a., 2017; Schilpzand et al., 2016) or hierarchy-attenuating versus hierarchy-enhancing professions (Aiello et al., 2018; Haley \& Sidanius, 2015; Pratto et al., 1994).

Another limitation of this study may have been common method bias (CMB), which is a potential source of measurement error that poses a threat to the relationships found between measures of different constructs (Reio, 2010). CMB occurs when the relationship between variables is inflated or deflated due to the use of self-report measures (Podsakoff et al., 2003; Richardson et. al, 2009; Spector, 2006). To minimize the likelihood of CMB, this study implemented the recommendations suggested by Reio (2010) by ensuring participant confidentiality, counterbalancing the order of the questions, and informing participants that there was no preferred or correct answer, and included a social desirability scale. Due to the potential of common method bias (CMB), data should be collected at different points in time and include Harman's diagnostic test to assess for the possible presence of CMB (Podsakoff et al., 2003; Reio, 2010).

The decision to use the Likert scales established in the literature was made to contribute to the literature based on these measures, but also to expand it by exploring new relationships between the measured concepts. At the same time, the Likert instrument might simplify the complexities working in the real world that give rise to 
uncivil behavior. As noted in the results chapter of this study, the measurement model constructed for the SEM analysis required the researcher to covary five pairs of intrascale error terms. Future studies should assess the possibility of a wording effect within the SDO7 (Ho et al., 2015) scale. Wording effect is another potential source of CMB, which may occur in scales that include positively and negatively worded items (Podsakoff et al., 2003). Scholars should consider testing the factor structure of the SDO7 scale with two potential approaches. One approach is the correlated trait-correlated method (CTCM), and the other approach is the correlated trait-correlated uniqueness (CTCU) method suggested by Xin \& Chi (2010) who tested the wording effect of an older social dominance scale. Future studies should test the direct relationship between social dominance orientation and instigated incivility by comparing alternative models based on the different measures of social dominance. Model 1 should include both dimensions of SDO as was done in the current study, Model 2 should include only SDOD, Model 3 should include only SDO-E, and Model 4 should treat SDO-D and SDO-E as a unidimensional measure. The current model included the measurement of instigated incivility as a second-order factor formed by three first-order latent variables that included (a) hostility, (b) exclusionary behavior, and (c) gossiping. Future research should consider testing the models on the specific sub-dimensions of incivility to explore various response trajectories pertaining to the different forms of uncivil behavior.

Special attention should be paid also to effects of witnessed and experienced incivility, which were not addressed in the current research. There is a relationship between witnessed incivility and instigated incivility (Cortina et al., 2017; Holm et al., 2015; Schilpzand et al., 2016). Witnessed incivility may be used as a control variable to 
improve the current model as it would help isolate the effect of SDO on instigated incivility. Similarly, experienced incivility is linked to instigated incivility (Anderson \& Pearson, 1999; Cortina et al., 2017; Francis et al., 2016; Schilpzand et al., 2016). Likewise, a measure of experienced incivility should be considered to further isolate the effect to SDO on instigated incivility.

Research on emotion regulation focuses on employee-customer interactions, leaving emotion regulation in employee-employee interactions relatively unexplored (Grandey \& Gabriel, 2015; Kim et al., 2013). Experimental longitudinal studies can assess the effect of the emotion regulation strategy of cognitive reappraisal on instigated incivility over time. This approach could include the assessment of a cognitive reappraisal training in reducing uncivil workplace behavior. There is a significant lack of empirically tested interventions for workplace incivility (Cortina et al., 2017), and cognitive reappraisal is an antecedent focused strategy of emotion regulation that occurs before emotion response tendencies have activated and have changed behavior and physiological responding (Gross, 1998b; Hochschild, 1983). Longitudinal studies can also address the issue of causality in research on uncivil workplace behavior.

The most current and comprehensive review of workplace incivility literature conducted by Schilpzand et al. (2016) presents antecedent variables related to instigated incivility that future studies should consider testing as potential mediators or moderators on the social dominance and incivility relationship. Two important antecedent variables are emotional exhaustion and depression. The results from this study indicate that emotional exhaustion and depression may be important distal outcomes for the model proposed in this study. In the current study, expressive suppression had an indirect effect 
that reduced instigated incivility among employees with strong SDO-D traits. Scholars have identified emotional exhaustion (Van Dijk \& Brown, 2006; Glomb \& Tews, 2004; Zapf \& Holz, 2006), and increased depressive symptoms (Gross, 2002; Gross \& John, 2003; John \& Gross, 2004) among individuals who habitually employ the emotion regulation strategy of expressive suppression. The proposed model should further assess how emotional exhaustion and depression impact organizational outcomes such as job performance and voluntary turnover.

A final recommendation for future research is to explore the nature of the relationships proposed in this study with a qualitative research design. This research has maintained its distance from the individual perpetrators and victims by utilizing survey methodology. An ethnographic approach which approaches the worksite experience of workers from the point of view of the participants and interrogates the behaviors of workers in specific industries in their relationship with incivility may yield different insights into the linkages between social dominant world views and specific triggers for incivility behavior. Rather than asking the participants what they did, an ethnographer studying incivility would fill notebooks recording what the participants actually were doing. An extended case study in a specific industry (Burawoy, 1998) would be particularly useful in digging deeper into the workplace patterns associated with uncivil workplace behavior. Similarly, ethnographic studies could follow the insights of Montgomery and colleagues (2004) who found that group level norms of civility and differences in observer perceptions of civility norm violations are based on the degree to which the observer identifies with the instigator and victim of incivility. Answering questions about identity and identification are beyond the scope of this work, but an 
extension of its research questions utilizing qualitative methodology would contribute to the field of workplace incivility research. For example, theorizing on Butler's work on identity as performance (Butler, 1990), an ethnographer can ask to what degree an instigator is motivated by the perceived bond of identity with other instigators and to what degree an act of incivility can be interpreted as a "performance" of solidarity based on identification with a generalized other. Because social dominance orientation is a cultural dimension of personality (Sidanius \& Pratto, 1999) which expands beyond the worksite, methodologies utilizing broad ranging interviews as well as participant observation could establish the link between broader cultural influences (class, gender, ethnicity/race), and workplace incivility behavior of social dominators. A final recommendation for future research is the implementation of a mixed methods research design. Combining both qualitative and quantitative approaches may likely offer more nuanced research findings and outcomes (Johnson, Onwuegbuzie, \& Turner, 2007). Human resource development research is conducted in complex organizational settings and in international contexts, which lend themselves to a mixed methods approach (Reio \& Werner, 2007).

\section{Conclusion}

The purpose of this study was to explore a parallel mediator model of instigated incivility moderated by workgroup civility climate norms. This research examined the influence of the mediation of the emotion regulation strategies of cognitive reappraisal and expressive suppression, on two types of social dominance orientation - support for intergroup dominance (SDO-D) and intergroup antiegalitarianism (SDO-E) - on the outcome of instigated incivility. The findings suggest that employees with strong SDO-D 
are likely to instigate incivility toward a coworker in both civil and uncivil workgroup civility climates. Further, this study found that the emotion regulation strategy of expressive suppression significantly reduced uncivil behaviors. These findings are consistent with previous research on social dominance orientation and expressive suppression of emotions, but contrast previous research on the negative social outcomes of expressive suppression. Future research should continue to test the role of emotion regulation strategies on instigated incivility as the additional findings from this study are consistent with previous research showing the positive outcomes of cognitive reappraisal on interpersonal behavior.

One of the primary goals within the field of HRD is to create and support healthy and productive work environments for all employees (Callahan, 2011; Estes \& Wang, 2008; Githens, 2011; Reio \& Sanders-Reio, 2011; Trudel \& Reio, 2011). This study moved beyond most workplace incivility studies, which focus on victims of workplace incivility, by exploring uncivil behavior from the perspective of the perpetrator. Perhaps the most significant finding in this study suggests that employees in this research with strong levels of intergroup dominance (SDO-D) were very likely to instigate workplace incivility - in both civil and uncivil workgroup climates. The HRD community has an ethical and moral obligation to address issues of social justice within organizations (Byrd, 2018). Expanding the research literature on instigated incivility is necessary for helping HRD professionals and managers act to reduce uncivil behavior within organizations (Cortina et al., 2017; Jex et al., 2010; Meier \& Semmer, 2012; Schilpzand et al., 2016). 


\section{REFERENCES}

Abas, N. A. H. B., \& Otto, K. (2016). Interpersonal mistreatment, organizational attitudes and well-being: The impact of instigator's hierarchical position and demographic characteristics. Organizational Management Journal, 13, 5-20. https://doi.org/10.1080/15416518.2015.1129307

Abler, B., \& Kessler, H. (2009). Emotion regulation questionnaire: A German version of the ERQ by Gross \& John. Diagnostica, 55, 144-152. https://doi.org/10.1026/0012-1924.55.3.144

Adams, G. A., \& Webster, J. R. (2013). Emotional regulation as a mediator between interpersonal mistreatment and distress. European Journal of Work and Organizational Psychology, 22(6), 697-710. https://doi.org/10.1080/1359432X.2012.698057

Aiello, A., Pratto, F., \& Pierro, A. (2013). Framing social dominance orientation and power in organizational context. Basic and Applied Social Psychology, 35, 487495. https://doi.org/10.1080/01973533.2013.823614

Aiello, A., Tesi, A., Pratto, F., \& Pierro, A. (2018). Social dominance and interpersonal power: Asymmetrical relationships within hierarchy-enhancing and hierarchyattenuating work environments. Journal of Applied Social Psychology, 48, 35-45. Retrieved from https://doi-org.ezproxy.fiu.edu/10.1111/jasp.12488 https://doi.org/10.1111/jasp.12488

Aker, A., El-Haj, M., Albakour, M.-D., \& Kruschwitz, U. (2012, May). Assessing crowdsourcing quality through objective tasks. Paper presented at the 8th International Conference on Language Resources and Evaluation, Istanbul, Turkey

Aldao, A., Nolen-Hoeksema, S., \& Schweizer, S. (2010). Emotion-regulation strategies across psychopathology: A meta-analytic review. Clinical Psychology Review, 30, 217-237. https://doi.org/10.1016/j.cpr.2009.11.004

Allen, J. A., Pugh, S. D., Grandey, A. A., \& Groth, M. (2010). Following display rules in good or bad faith?: Customer orientation as a moderator of the display ruleemotional labor relationship. Human Performance, 23, 101-115. https://doi.org/10.1080/08959281003621695

Altemeyer, B. (1988). Enemies of freedom. Understanding right-wing authoritarianism. San Francisco, CA: Jossey-Bass. 
Amir, O., Rand, D. G., \& Gal, Y. K. (2012). Economic games on the internet: The effect of \$1 stakes. PLoS One, 7(2), e31461.

https://doi.org/10.1371/journal.pone.0031461

Andersson, L. M., \& Pearson, C. M. (1999). Tit for tat? The spiraling effect of incivility in the workplace. Academy of Management Review, 24, 452-471.

https://doi.org/10.5465/amr.1999.2202131

Angie, A. D., Connelly, S., Waples, E. P., \& Kligyte, V. (2011). The influence of discrete emotions on judgement and decision-making: A meta-analytic review. Cognition and Emotion, 25(8), 1393-1422. Retrieved from https://doi-

org.ezproxy.fiu.edu/10.1080/02699931.2010.550751

https://doi.org/10.1080/02699931.2010.550751

Appleton, A. A., \& Kubzansky, L. D. (2014). Emotion regulation and cardiovascular disease risk. In J. J. Gross (Ed.), Handbook of emotion regulation (2nd ed., pp. 596-612). New York, NY: Guilford.

Arbuckle, J. L. (2014). Amos 23.0 User's Guide. Chicago: IBM SPSS.

Arneson, R. J. (2013). Equality of opportunity: Derivative not fundamental. Journal of Social Philosophy, 44, 316-330. https://doi.org/10.1111/josp.12036

Averill, J. R. (1980). On the paucity of positive emotions. In K. R. Blankstein, P. Pliner, \& J. Polivy (Eds.), Advances in the study of communication and affect (Vol. 6, pp. 7-45). New York: Plenum.

Bachorowski, J. A., \& Braaten, E. B. (1994). Emotional intensity: Measurement and theoretical implications. Personality and Individual Differences, 17, 191-199. https://doi.org/10.1016/0191-8869(94)90025-6

Baker, R., Blumberg, S. J., Brick, J. M., Couper, M. P., Courtright, M., Dennis, J. M., . . . Zahs, D. (2010). Research synthesis: AAPOR report on online panels. Public Opinion Quarterly, 74, 711-781. https://doi.org/10.1093/poq/nfq048

Balzarotti, S., John, O. P., \& Gross, J. J. (2010). An Italian adaptation of the Emotion Regulation Questionnaire. European Journal of Psychological Assessment, 26, 61-67. https://doi.org/10.1027/1015-5759/a000009

Baron, R. M., \& Kenny, D. A. (1986). The moderator-mediator variable distinction in social psychological research: Conceptual, strategic, and statistical considerations. Journal of Personality and Social Psychology, 51(6), 1173-1182.

https://doi.org/10.1037/0022-3514.51.6.1173 
Barrett, L. F., Mesquita, B., Ochsner, K. N., \& Gross, J. J. (2007). The experience of emotion. Annual Review of Psychology, 58, 373-403.

https://doi.org/10.1146/annurev.psych.58.110405.085709

Barrick, M. R., Stewart, G. L., \& Piotrowski, M. (2002). Personality and job performance: Test of the mediating effects of motivation among sales representatives. The Journal of Applied Psychology, 87, 43-51. Retrieved from http://dx.doi.org.ezproxy.fiu.edu/10.1037/0021-9010.87.1.43 https://doi.org/10.1037/0021-9010.87.1.43

Barsade, S. G., \& Gibson, D. E. (2012). Group affect: Its influence on individual and group outcomes. Current Directions in Psychological Science, 21, 119-123. https://doi.org/10.1177/0963721412438352

Bartneck, C., Duenser, A., Moltchanova, E., \& Zawieska, K. (2015). Comparing the similarity of responses received from studies in Amazon's Mechanical Turk to studies conducted online and with direct recruitment. PLoS One, 10(4), e0121595. Retrieved from http://link.galegroup.com.ezproxy.fiu.edu/apps/doc/A425892518/HRCA?u=miam 11506\&sid=HRCA\&xid=b2fd85d6 https://doi.org/10.1371/journal.pone.0121595

Batalha, L., Reynolds, K. J., \& Newbigin, C. A. (2011). All else being equal: Are men always higher in social dominance orientation than women? European Journal of Social Psychology, 41, 796-806. https://doi.org/10.1002/ejsp.829

Bates, C., \& Heaven, P. C. (2001). Attitudes to women in society: The role of social dominance orientation and social values. Journal of Community \& Applied Social Psychology, 11, 43-49. https://doi.org/10.1002/casp.589

Bennett, R. J., \& Robinson, S. L. (2000). Development of a measure of workplace deviance. Journal of Applied Psychology, 85, 349 - 360. doi:10.I037//00219010.85.3.349

Bergh, R., Sidanius, J., \& Sibley, C. G. (2015). Dimensions of social dominance: Their personality and socio-political correlates within a New Zealand probability sample. New Zealand Journal of Psychology, 44, 25-34. Retrieved from http://www.psychology.org.nz/wp-content/uploads/72176-NZJP-Vol-44-No2_Social-Dominance.pdf

Berinsky, A. J., Huber, G. A., \& Lenz, G. S. (2012). Evaluating online labor markets for experimental research: Amazon.com's Mechanical Turk. Political Analysis, 20, 351-368. https://doi.org/10.1093/pan/mpr057

Bieri, J. (1955). Cognitive complexity-simplicity and predictive behavior. Journal of Abnormal and Social Psychology, 51, 263-268. Retrieved from 
http://dx.doi.org.ezproxy.fiu.edu/10.1037/h0043308

https://doi.org/10.1037/h0043308

Binggeli, S., Krings, F., \& Sczesny, S. (2014). Stereotype content associated with immigrant groups in Switzerland. Swiss Journal of Psychology, 73, 123-133. https://doi.org/10.1024/1421-0185/a000133

Birkeland, I. K., \& Nerstad, C. (2016). Incivility is (not) the very essence of love: Passion for work and incivility instigation. Journal of Occupational Health Psychology, 21, 77-90. Retrieved from http://dx.doi.org.ezproxy.fiu.edu/10.1037/a0039389 https://doi.org/10.1037/a0039389

Blau, G. (2007). Partially testing a process model for understanding victim responses to an anticipated worksite closure. Journal of Vocational Behavior, 71, 401- 428. doi:1010.1016/j.jvb.2007.08.005

Blau, G., \& Andersson, L. (2005). Testing a measure if instigated workplace incivility. Journal of Occupational and Organizational Psychology, 78, 595-614. https://doi.org/10.1348/096317905X26822

Brownridge, D. A., \& Halli, S. S. (2002). Double jeopardy?: Violence against immigrant women in Canada. Violence and Victims, 17, 455-471. Retrieved from http://ezproxy.fiu.edu/login?url=https://search-proquestcom.ezproxy.fiu.edu/docview/208555710?accountid=10901 https://doi.org/10.1891/vivi.17.4.455.33680

Brunetta, F., \& Reio, T. (2016). How welcome are we? Immigrants as targets of uncivil behavior. In R. Mizzi, T. Rocco, \& S. Shore (Eds.), Disrupting adult and community education: Teaching, learning, and working in the periphery (pp. 227-242). Albany, NY: SUNY Press.

Buhrmester, M., Kwang, T., \& Gosling, S. D. (2011). Amazon's Mechanical Turk: A new source of cheap, yet high-quality, data? Perspectives on Psychological Science, 6, 3-5. https://doi.org/10.1177/1745691610393980

Bunk, J. A., \& Magley, V. J. (2013). The role of appraisals and emotions in understanding experiences of workplace incivility. Journal of Occupational Health Psychology, 18, 87-105. https://doi.org/10.1037/a0030987

Burawoy, M. (1998). The extended case method. Sociological Theory, 16, 4-33. https://doi.org/10.1111/0735-2751.00040

Burkitt, I. (2013). Self and others in the field of perception: The role of micro-dialogue, feeling, and emotion in perception. Journal of Theoretical and Philosophical Psychology, 33, 267-269. Retrieved from 
http://dx.doi.org.ezproxy.fiu.edu/10.1037/a0030255

https://doi.org/10.1037/a0030255

Bushman, B. J., Bonacci, A. M., Pedersen, W. C., Vasquez, E. A., \& Miller, N. (2005). Chewing on it can chew you up: Effects of rumination on triggered displaced aggression. Journal of Personality and Social Psychology, 88, 969-983. https://doi.org/10.1037/0022-3514.88.6.969

Butler, E. A., Egloff, B., Wilhelm, F. H., Smith, N. C., Erickson, E. A., \& Gross, J. J. (2003). The social consequences of expressive suppression. Emotion (Washington, D.C.), 3, 48-67. https://doi.org/10.1037/1528-3542.3.1.48

Butler, E. A., Gross, J. J., \& Barnard, K. (2014). Testing the effects of suppression and reappraisal on emotional concordance using a multivariate multilevel model. Biological Psychology, 98, 6-18. https://doi.org/10.1016/j.biopsycho.2013.09.003

Butler, J. (1990). Gender trouble and the subversion of identity. New York, London: Routledge.

Byrd, M. Y. (2018). Does HRD have a moral duty to respond to matters of social injustice? Human Resource Development International, 21, 3-11. https://doi.org/10.1080/13678868.2017.1344419

Callahan, J. L. (2011). Incivility as an instrument of oppression: Exploring the role of power in constructions of civility. Advances in Developing Human Resources, 13, 10-21. https://doi.org/10.1177/1523422311410644

Callegaro, M., Baker, R. P., Bethlehem, J., Göritz, A. S., Krosnick, J. A., \& Lavrakas, P. J. (2014). A critical review of studies investigating the quality of data obtained with online panels based on probability and nonprobability samples. In Callegaro, ... (Eds.), Online panel research: A data quality perspective (pp. 23-53). United Kingdom: John Wiley \& Sons. https://doi.org/10.1002/9781118763520.ch2

Caricati, L. (2007). The relationship between social dominance orientation and gender: The mediating role of social values. Sex Roles, 57(3-4), 159-171. https://doi.org/10.1007/s11199-007-9231-3

Caza, B. B., \& Cortina, L. M. (2007). From insult to injury: Explaining the impact of incivility. Basic and Applied Social Psychology, 29, 335-350. https://doi.org/10.1080/01973530701665108

Chan, C., \& Holosko, M. J. (2016a). An overview of the use of Mechanical Turk in behavioral sciences: Implications for social work. Research on Social Work Practice, 26, 441-448. https://doi.org/10.1177/1049731515594024 
Chan, C., \& Holosko, M. J. (2016b). A review of information and communication technology enhanced social work interventions. Research on Social Work Practice, 26, 88-100. https://doi.org/10.1177/1049731515578884

Chandler, J., Mueller, P., \& Paolacci, G. (2014). Nonnaïveté among Amazon Mechanical Turk workers: Consequences and solutions for behavioral researchers. Behavior Research Methods, 46, 112-130. https://doi.org/10.3758/s13428-013-0365-7

Chen, L. J., \& Hung, H. C. (2016). The indirect effect in multiple mediators model by structural equation modeling. European Journal of Business. Economics and Accountancy, 4, 36-43.

Chen, Y., Ferris, D. L., Kwan, H. K., Yan, M., Zhou, M., \& Hong, Y. (2013). Self-love's lost labor: A self-enhancement model of workplace incivility. Academy of Management Journal, 56, 1199-1219. https://doi.org/10.5465/amj.2010.0906

Chiaburu, D. S., Oh, I. S., Berry, C. M., Li, N., \& Gardner, R. G. (2011). The five-factor model of personality traits and organizational citizenship behaviors: A metaanalysis. The Journal of Applied Psychology, 96, 1140-1166. https://doi.org/10.1037/a0024004

Chui, C. W. S., \& Dietz, J. (2014). Observing workplace incivility towards women: The roles of target reactions, actor motives, and actor-target relationships. Sex Roles, 71, 95-108. https://doi.org/10.1007/s11199-014-0367-7

Chunara, R., Chhaya, V., Bane, S., Mekaru, S. R., Chan, E. H., Freifeld, C. C., \& Brownstein, J. S. (2012). Online reporting for malaria surveillance using micromonetary incentives, in urban India 2010-2011. Malaria Journal, 11(43), 43. https://doi.org/10.1186/1475-2875-11-43

Clore, G. L., \& Tamir, M. (2002). Affect as embodied information. Psychological Inquiry, 13, 37-45. Retrieved from http://www.jstor.org/stable/1449537

Cortina, L. M. (2008). Unseen injustice: Incivility as modern discrimination in organizations. Academy of Management Review, 33, 55-75. https://doi.org/10.5465/amr.2008.27745097

Cortina, L. M., Kabat-Farr, D., Leskinen, E. A., Huerta, M., \& Magley, V. J. (2013). Selective incivility as modern discrimination in organizations: Evidence and impact. Journal of Management, 39, 1579-1605. https://doi.org/10.1177/0149206311418835

Cortina, L. M., Kabat-Farr, D., Magley, V. J., \& Nelson, K. (2017). Researching rudeness: The past, present, and future of the science of incivility. Journal of Occupational Health Psychology, 22, 299-313. Retrieved from 
http://dx.doi.org.ezproxy.fiu.edu/10.1037/ocp0000089

https://doi.org/10.1037/ocp0000089

Cortina, L. M., Lonsway, K. L., Magley, V. J., Freeman, L. V., Collinsworth, L. L., Hunter, M., \& Fitzgerald, L. F. (2002). What's gender got to do with it? Incivility in the federal courts. Law \& Social Inquiry, 27, 235-270.

https://doi.org/10.1111/j.1747-4469.2002.tb00804.x

Cortina, L. M., \& Magley, V. J. (2009). Patterns and profiles of response to incivility in the workplace. Journal of Occupational Health Psychology, 14, 272-288. https://doi.org/10.1037/a0014934

Cortina, L. M., Magley, V. J., Williams, J. H., \& Langhout, R. D. (2001). Incivility in the workplace: Incidence and impact. Journal of Occupational Health Psychology, 6, 64-80. Retrieved from http://dx.doi.org.ezproxy.fiu.edu/10.1037/1076-

8998.6.1.64 https://doi.org/10.1037/1076-8998.6.1.64

Cortina, L. M., Rabelo, V. C., \& Holland, K. J. (2018). Beyond blaming the victim: Toward a more progressive understanding of workplace mistreatment. Industrial and Organizational Psychology: Perspectives on Science and Practice, 11, 81100. https://doi.org/10.1017/iop.2017.54

Costa, P. T., Jr., \& McCrae, R. R. (1992). Four ways five factors are basic. Personality and Individual Differences, 13, 653-665. https://doi.org/10.1016/01918869(92)90236-I

Crampton, S. M., \& Hodge, J. W. (2008). Rudeness and incivility in the workplace. Journal of Leadership, Accountability and Ethics, Vol. Fall, 41- 48. Retrieved from https://search.proquest.com/openview/1a942cc6ab1dafda4549231ec53c5ed0/1?pq -origsite $=$ gscholar\&cbl $=39006$

Crawford, J. T., \& Pilanski, J. M. (2014). Political intolerance, right and left. Political Psychology, 35, 841-851. https://doi.org/10.1111/j.1467-9221.2012.00926.x

Creswell, J. W. (2014). Research design: Qualitative, quantitative, and mixed methods approaches. Thousand Oaks, CA: Sage publications.

Dambrun, M., Duarte, S., \& Guimond, S. (2004). Why are men more likely to support group-based dominance than women? The mediating role of gender identification. British Journal of Social Psychology, 43(Pt 2), 287-297. https://doi.org/10.1348/0144666041501714

Daniels, S. R., Simmons, A., \& Hall, A. V. (2015). Incivility perpetration: understanding the role of social dominance orientation, psychological entitlement, and moral 
disengagement. In G. Thrasher (Chair), Toward a better understanding of workplace mistreatment. Symposium conducted at the meeting of Academy of Management Meeting, Vancouver, BC, Canada.

Denny, B. T., \& Ochsner, K. N. (2014). Behavioral effects of longitudinal training in cognitive reappraisal. Emotion (Washington, D.C.), 14, 425-433. https://doi.org/10.1037/a0035276

Dasgupta, N., Desteno, D., Williams, L. A., \& Hunsinger, M. (2009). Fanning the flames of prejudice: The influence of specific incidental emotions on implicit prejudice. Emotion (Washington, D.C.), 9(4), 585-591. Retrieved from http://dx.doi.org.ezproxy.fiu.edu/10.1037/a0015961 https://doi.org/10.1037/a0015961

Davidov, G. (2005). Who is a worker? Industrial Law Journal, 34, 57-71. https://doi.org/10.1093/ilj/34.1.57

Denton, L. T., Campbell, C., \& Johnson, C. D. (2009). Dementors in our midst: Managing the highly productive but morale-killing employee. The Journal of Applied Management and Entrepreneurship, 14, 3-25. Retrieved from http://ezproxy.fiu.edu/login?url=https://search-proquestcom.ezproxy.fiu.edu/docview/203914081?accountid=10901

Diefendorff, J. M., \& Croyle, M. H. (2008). Antecedents of emotional display rule commitment. Human Performance, 21, 310-332. https://doi.org/10.1080/08959280802137911

Diefendorff, J. M., Croyle, M. H., \& Gosserand, R. H. (2005). The dimensionality and antecedents of emotional labor strategies. Journal of Vocational Behavior, 66, 339-357. https://doi.org/10.1016/j.jvb.2004.02.001

Dillman, D. A. (2000). Mail and internet surveys: The tailored design method (2nd ed.). New York: John Wiley \& Sons.

Dillman, D. A., Smyth, J. D., \& Christian, L. M. (2009). Internet, mail, and mixed-mode survey: The tailored design method. Hoboken, NJ: John Wiley \& Sons.

Dollard, J., Doob, L. W., Miller, N. E., Mowrer, O. H., \& Sears, R. R. (1939). Frustration and aggression. New Haven, CT: Yale University Press. https://doi.org/10.1037/10022-000

Douglas, S. C., \& Martinko, M. J. (2001). Exploring the role of individual differences in the prediction of workplace aggression. The Journal of Applied Psychology, 86, 547-559. https://doi.org/10.1037/0021-9010.86.4.547 
Dovidio, J. F., \& Gaertner, S. L. (1998). On the nature of contemporary prejudice: The causes, consequences, and challenges of aversive racism. In J. L. Eberhardt \& S. T. Fiske (Eds.), Confronting racism: The problem and the response (pp. 3-32). Thousand Oaks, CA: Sage.

Duckitt, J., \& Sibley, C. G. (2007). Right wing authoritarianism, social dominance orientation and the dimensions of generalized prejudice. European Journal of Personality, 21, 113-130. https://doi.org/10.1002/per.614

Duriez, B., \& Van Hiel, A. (2002). The march of modern fascism. A comparison of social dominance orientation and authoritarianism. Personality and Individual Differences, 32, 1199-1213. https://doi.org/10.1016/S0191-8869(01)00086-1

Dworkin, R. (1981). What is equality? Part 2: Equality of resources. Philosophy \& Public Affairs, 10, 283-245.

Einarsen, S. (1999). The nature and causes of bullying at work. International Journal of Manpower, 20, 16 -27. http://dx.doi.org/10.1108/0143 7729910268588

Einarsen, S., Raknes, B. I., \& Matthiesen, S. B. (1994). Bullying and harassment at work and their relationship to work environment quality: An exploratory study. European Work and Organizational Psychologist, 4, 381-401. https://doi.org/10.1080/13594329408410497

Ekman, P. (1973). Cross-cultural studies of facial expression. In P. Ekman (Ed.), Darwin and facial expression: A century of research in review (pp. 169-222). New York: Academic Press.

Elias, N. (1978). The history of manners. Oxford: Blackwell.

English, T., \& John, O. P. (2013). Understanding the social effects of emotion regulation: The mediating role of authenticity for individual differences in suppression. Emotion (Washington, D.C.), 13, 314-329. https://doi.org/10.1037/a0029847

Estes, B., \& Wang, J. (2008). Workplace incivility: Impacts on individual and organizational performance. Human Resource Development Review, 7, 218-240. https://doi.org/10.1177/1534484308315565

Feather, N. T. (2004). Value correlates of ambivalent attitudes toward gender relations. Personality and Social Psychology Bulletin, 30, 3-12. https://doi.org/10.1177/0146167203258825

Feather, N. T., \& McKee, I. R. (2012). Values, right-wing authoritarianism, social dominance orientation, and ambivalent attitudes toward women. Journal of 
Applied Social Psychology, 42, 2479-2504. https://doi.org/10.1111/j.15591816.2012.00950.x

Ferris, G. R., Zinko, R., Brouer, R. L., Buckley, M. R., \& Harvey, M. G. (2007). Strategic bullying as a supplementary, balanced perspective on destructive leadership. The Leadership Quarterly, 18, 195-206. https://doi.org/10.1016/j.leaqua.2007.03.004

Field, A., \& Hole, G. (2003). How to design and report experiments. London: Sage.

Fiske, S. T. (1998). Stereotyping, prejudice, and discrimination. In D. T. Gilbert, S. T. Fiske, \& G. Lindzey (Eds.), Handbook of social psychology (4th ed., Vol. 2, pp. 357-411). New York: McGrawHill.

Fisher, R. A. (1921). On the "probable error" of a coefficient of correlation deduced from a small sample. Metron, 1, 3-32.

Foels, R., \& Pappas, C. J. (2004). Learning and unlearning the myths we are taught: Gender and social dominance orientation. Sex Roles, 50, 743-757. https://doi.org/10.1023/B:SERS.0000029094.25107.d6

Foels, R., \& Reid, L. D. (2010). Gender differences in social dominance orientation: The role of cognitive complexity. Sex Roles, 62, 684-692. https://doi.org/10.1007/s11199-010-9775-5

Fornell, C., \& Larcker, D. F. (1981). Evaluating Structural Equation Models with Unobservable Variables and Measurement Error. JMR, Journal of Marketing Research, 18, 39-50. https://doi.org/10.2307/3151312

Fox, S., \& Stallworth, L. E. (2005). Racial/ethnic bullying: Exploring links between bullying and racism in the US workplace. Journal of Vocational Behavior, 66, 438-456. doi:10.1016/j.jvb.2004. 01.002

Francis, L., Holmvall, C. M., \& O'Brien, L. E. (2015). The influence of workload and civility of treatment on the perpetration of email incivility. Computers in Human Behavior, 46, 191-201. https://doi.org/10.1016/j.chb.2014.12.044

French, J. R., Jr. (1956). A formal theory of social power. Psychological Review, 63, 181-194. https://doi.org/10.1037/h0046123

Gabriel, A. S., Daniels, M. A., Diefendorff, J. M., \& Greguras, G. J. (2015). Emotional labor actors: A latent profile analysis of emotional labor strategies. The Journal of Applied Psychology, 100, 863-879. https://doi.org/10.1037/a0037408 
Gall, M. D., Gall, J. P., \& Borg, W. R. (2007). Educational research: An introduction (8th ed.). Boston: Allyn and Bacon.

Gallus, J. A., Bunk, J. A., Matthews, R. A., Barnes-Farrell, J. L., \& Magley, V. J. (2014). An eye for an eye? Exploring the relationship between workplace incivility experiences and perpetration. Journal of Occupational Health Psychology, 19, 143-154. Retrieved from http://dx.doi.org/10.1037/a0035931 https://doi.org/10.1037/a0035931

Geuens, M., \& De Pelsmacker, P. (2002). Developing a short affect intensity scale. Psychological Reports, 91, 657-670. https://doi.org/10.2466/pr0.2002.91.2.657

Ghosh, R., Dierkes, S., \& Falletta, S. (2011). Incivility spiral in mentoring relationships: Reconceptualizing negative mentoring as deviant workplace behavior. Advances in Developing Human Resources, 13, 22-39. https://doi.org/10.1177/1523422311410639

Gianaros, P. J., Marsland, A. L., Kuan, D. C. H., Schirda, B. L., Jennings, J. R., Sheu, L. K., . . Manuck, S. B. (2014). An inflammatory pathway links atherosclerotic cardiovascular disease risk to neural activity evoked by the cognitive regulation of emotion. Biological Psychiatry, 75, 738-745.

https://doi.org/10.1016/j.biopsych.2013.10.012

Gill, M. J., \& Sypher, B. D. (2009). Workplace incivility and organizational trust. In P. Lutgen-Sandvik \& B. D. Sypher (Eds.), Destructive organizational communication: Processes, consequences, and constructive ways of organizing (pp. 53-73). New York, NY: Routledge.

Githens, R. P. (2011). Diversity and incivility: Toward an action-oriented approach. Advances in Developing Human Resources, 13, 40-53. https://doi.org/10.1177/1523422311410646

Glick, P., \& Fiske, S. T. (1996). The Ambivalent Sexism Inventory: Differentiating hostile and benevolent sexism. Journal of Personality and Social Psychology, 70, 491-512. https://doi.org/10.1037/0022-3514.70.3.491

Glomb, T. M., \& Liao, H. (2003). Interpersonal aggression in work groups: Social influence, reciprocal, and individual effects. Academy of Management Journal, $46,486-496$.

Glomb, T. M., \& Tews, M. J. (2004). Emotional labor: A conceptualization and scale development. Journal of Vocational Behavior, 64, 1-23. https://doi.org/10.1016/S0001-8791(03)00038-1 
Goleman, D. (2006). Social intelligence: The new science of human relationships. New York: Bantam Books.

Goodman, J. K., Cryder, C. E., \& Cheema, A. (2013). Data collection in a flat world: The strengths and weaknesses of Mechanical Turk samples. Journal of Behavioral Decision Making, 26, 213-224. https://doi.org/10.1002/bdm.1753

Gosling, S. D., \& Mason, W. (2015). Internet research in psychology. Annual Review of Psychology, 66, 877-902. https://doi.org/10.1146/annurev-psych-010814-015321

Grandey, A. A. (2000). Emotion regulation in the workplace: A new way to conceptualize emotional labor. Journal of Occupational Health Psychology, 5, 95-110. https://doi.org/10.1037/1076-8998.5.1.95

Grandey, A. A. (2003). When "the show must go on": Surface acting and deep acting as determinants of emotional exhaustion and peer-rated service delivery. Academy of Management Journal, 46, 86-96. https://doi.org/10.2307/30040678

Grandey, A. A., Dickter, D. N., \& Sin, H. P. (2004). The customer is not always right: Customer aggression and emotion regulation of service employees. Journal of Organizational Behavior, 25, 397-418. https://doi.org/10.1002/job.252

Grandey, A. A., Diefendorff, J., \& Rupp, D. E. (Eds.). (2013). Emotional labor in the 21 st century: Diverse perspectives on emotion regulation at work. New York: Routledge. https://doi.org/10.4324/9780203100851

Grandey, A. A., \& Gabriel, A. S. (2015). Emotional labor at a crossroads: Where do we go from here? Annual Review of Organizational Psychology and Organizational Behavior, 2, 323-349. https://doi.org/10.1146/annurev-orgpsych-032414-111400

Grandey, A. A., \& Melloy, R. C. (2017). The state of the heart: Emotional labor as emotion regulation reviewed and revised. Journal of Occupational Health Psychology, 22, 407-422. Retrieved from http://dx.doi.org.ezproxy.fiu.edu/10.1037/ocp0000067 https://doi.org/10.1037/ocp0000067

Gray, C. J., Carter, N. T., \& Sears, K. L. (2017). The UWBQ-I: An adaption and validation of a measure of instigated incivility. Journal of Business and Psychology, 32, 21-39. https://doi.org/10.1007/s10869-015-9433-6

Green, S. B. (1991). How many subjects does it take to do a regression analysis. Multivariate Behavioral Research, 26, 499-510. https://doi.org/10.1207/s15327906mbr2603_7 
Grewal, R., Cote, J. A., \& Baumgartner, H. (2004). Multicollinearity and measurement error in structural equation models: Implications for theory testing. Marketing Science, 23, 519-529. https://doi.org/10.1287/mksc.1040.0070

Gross, J. J. (1998a). Antecedent- and response-focused emotion regulation: Divergent consequences for experience, expression, and physiology. Journal of Personality and Social Psychology, 74, 224-237. https://doi.org/10.1037/0022-3514.74.1.224

Gross, J. J. (1998b). The emerging field of emotion regulation: An integrative review. Review of General Psychology, 2, 271-299. https://doi.org/10.1037/10892680.2.3.271

Gross, J. J. (2001). Emotion regulation in adulthood: Timing is everything. Current Directions in Psychological Science, 10, 214-219. https://doi.org/10.1111/14678721.00152

Gross, J. J. (2002). Emotion regulation: Affective, cognitive, and social consequences. Psychophysiology, 39, 281-291. https://doi.org/10.1017/S0048577201393198

Gross, J. J. (2013). Emotion regulation: Taking stock and moving forward. Emotion (Washington, D.C.), 13, 359-365. Retrieved from http://dx.doi.org.ezproxy.fiu.edu/10.1037/a0032135 https://doi.org/10.1037/a0032135

Gross, J. J. (2014). Emotion regulation: Conceptual and empirical foundations. In J. J. Gross (Ed.), Handbook of emotion regulation (pp. 3-20). New York, NY, US: Guilford Press.

Gross, J. J., \& John, O. P. (2003). Individual differences in two emotion regulation processes: Implications for affect, relationships, and well-being. Journal of Personality and Social Psychology, 85, 348-362. https://doi.org/10.1037/00223514.85.2.348

Gross, J. J., \& Levenson, R. W. (1993). Emotional suppression: Physiology, self-report, and expressive behavior. Journal of Personality and Social Psychology, 64, 970986. https://doi.org/10.1037/0022-3514.64.6.970

Gross, J. J., \& Levenson, R. W. (1997). Hiding feelings: The acute effects of inhibiting negative and positive emotion. Journal of Abnormal Psychology, 106, 95-103. https://doi.org/10.1037/0021-843X.106.1.95

Gross, J. J., \& Muñoz, R. F. (1995). Emotion regulation and mental health. Clinical Psychology: Science and Practice, 2, 151-164. https://doi.org/10.1111/j.14682850.1995.tb00036.x 
Gross, J. J., Richards, J. M., \& John, O. P. (2006). Emotion regulation in everyday life. In D. K. Snyder, J. Simpson, \& J. N. Hughes (Eds.), Emotion regulation in couples and families: Pathways to dysfunction and health (pp. 13-35). Washington, DC, US: American Psychological Association. https://doi.org/10.1037/11468-001

Gross, J. J., \& Thompson, R. A. (2007). Emotion regulation: Conceptual foundations. In J. J. Gross (Ed.), Handbook of emotion regulation (pp. 3-24). New York, NY, US: Guilford Press.

Gutek, B. A., Groth, M., \& Cherry, B. (2002). Achieving service success through relationships and enhanced encounters. The Academy of Management Executive, 16, 132-144. https://doi.org/10.5465/AME.2002.8951340

Häfner, M., \& Ijzerman, H. (2011). The face of love: Spontaneous accommodation as social emotion regulation. Personality and Social Psychology Bulletin, 37, 15511563. https://doi.org/10.1177/0146167211415629

Hair, J., Black, W., Babin, B., \& Anderson, R. (2010). Multivariate data analysis (7th ed.). Upper Saddle River, NJ, USA: Prentice-Hall, Inc.

Haley, H., \& Sidanius, J. (2005). Person-organization congruence and the maintenance of group-based social hierarchy: A social dominance perspective. Group Processes \& Intergroup Relations, 8, 187-203. https://doi.org/10.1177/1368430205051067

Harcourt, B. E. (2012). The politics of incivility. Arizona Law Review, 54, 345-373. Retrieved from http://heinonline.org/HOL/LandingPage?handle=hein.journals/arz54\&div=16\&id $=\&$ page $=$

Hauser, D. J., \& Schwarz, N. (2016). Attentive Turkers: MTurk participants perform better on online attention checks than do subject pool participants. Behavior Research Methods, 48, 400-407. https://doi.org/10.3758/s13428-015-0578-z

Hayes, A. F. (2009). Beyond Baron and Kenny: Statistical mediation analysis in the new millennium. Communication Monographs, 76, 408-420. https://doi.org/10.1080/03637750903310360

Hershcovis, S. (2011). 'Incivility, social undermining, bullying. . .Oh my!': A call to reconcile constructs within workplace aggression research. Journal of Organizational Behavior, 32, 499-519. https://doi.org/10.1002/job.689

Hershcovis, M. S., Turner, N., Barling, J., Arnold, K. A., Dupré, K. E., Inness, M., . . Sivanathan, N. (2007). Predicting workplace aggression: A meta-analysis. The Journal of Applied Psychology, 92, 228-238. https://doi.org/10.1037/00219010.92.1.228 
Hiel, A. V., \& Mervielde, I. (2002). Explaining conservative beliefs and political preferences: A comparison of social dominance orientation and authoritarianism. Journal of Applied Social Psychology, 32, 965-976. https://doi.org/10.1111/j.1559-1816.2002.tb00250.x

Hindricks, P., Verkuyten, M., \& Coenders, M. (2014). Dimensions of social dominance orientation: The roles of legitimizing myths and national identification. European Journal of Personality, 28, 538-549. https://doi.org/10.1002/per.1955

Hinkle, D. E., Wiersma, W., \& Jurs, S. G. (2003). Applied statistics for the behavioral sciences. Boston, MA: Houghton Mifflin Company.

Ho, A. K., Sidanius, J., Kteily, N., Sheehy-Skeffington, J., Pratto, F., Henkel, K. E., . . . Stewart, A. L. (2015). The nature of social dominance orientation: Theorizing and measuring preferences for intergroup inequality using the new $\mathrm{SDO}_{7}$ scale.

Journal of Personality and Social Psychology, 109, 1003-1028. https://doi.org/10.1037/pspi0000033

Ho, A. K., Sidanius, J., Pratto, F., Levin, S., Thomsen, L., Kteily, N., \& SheehySkeffington, J. (2012). Social dominance orientation: Revisiting the structure and function of a variable predicting social and political attitudes. Personality and Social Psychology Bulletin, 38, 583-606. https://doi.org/10.1177/0146167211432765

Hochschild, A. R. (1983). The managed heart: Commercialization of human feeling. Berkeley, CA: University of California Press.

Hodson, G., \& Costello, K. (2007). Interpersonal disgust, ideological orientations, and dehumanization as predictors of intergroup attitudes. Psychological Science, 18, 691-698. https://doi.org/10.1111/j.1467-9280.2007.01962.x

Holm, K., Torkelson, E., \& Bäckström, M. (2015). Models of workplace incivility: The relationships to instigated incivility and negative outcomes. BioMed Research International, 2015, 920239. https://doi.org/10.1155/2015/920239

Hooper, D., Coughlan, J., \& Mullen, M. R. (2008). Structural equation modeling: Guidelines for determining model fit. Electronic Journal of Business Research Methods, 6, 53-60. Retrieved from http://eds.b.ebscohost.com.ezproxy.fiu.edu/ehost/pdfviewer/pdfviewer?vid=2\&sid $=4 \mathrm{fc} 1 \mathrm{c} 703-\mathrm{fcff}-4566-8749-\mathrm{d} 3986 \mathrm{~d} 4 \mathrm{~d} 78 \mathrm{c} 8 \% 40$ sessionmgr 103

Horton, J. J., Rand, D. G., \& Zeckhauser, R. J. (2011). The online laboratory: Conducting experiments in a real labor market. Experimental Economics, 14, 399-425. https://doi.org/10.1007/s10683-011-9273-9 
Howe, J. (2006). The rise of crowdsourcing. Wired Magazine, 14(6), 1 - 5. Retrieved from http://www.wired.com/wired/archive/14.06/crowds.html

Hu, L., \& Liu, Y. (2017). Abuse for status: A social dominance perspective of abusive supervision. Human Resource Management Review, 27, 328-337. https://doi.org/10.1016/j.hrmr.2016.06.002

Huff, C., Tingley, D. (2015). "Who are these people?" Evaluating the demographic and political preferences of MTurk survey respondents. Research and Politics, JulySeptember 2015, 1-12. doi:10.1177/2053168015604648.

Hülsheger, U. R., \& Schewe, A. F. (2011). On the costs and benefits of emotional labor: A meta-analysis of three decades of research. Journal of Occupational Health Psychology, 16, 361-389. Retrieved from http://dx.doi.org.ezproxy.fiu.edu/10.1037/a0022876 https://doi.org/10.1037/a0022876

Ipeirotis, P. G., Provost, F., \& Wang, J. (2010). Quality management on Amazon Mechanical Turk. In Proceedings of the ACM SIGKDD Workshop on Human Computation (pp. 64-67). New York: ACM; Retrieved from https://dl.acm.org/citation.cfm?id=1837906 https://doi.org/10.1145/1837885.1837906

Jehn, K. A., Northcraft, G. B., \& Neale, M. A. (1999). Why differences make a difference: A field study of diversity, conflict, and performance in workgroups. Administrative Science Quarterly, 44, 741-763. https://doi.org/10.2307/2667054

Jex, S. M., Geimer, J. L., Clark, O., Guidroz, A. M., \& Yugo, J. E. (2010). Challenges and Recommendations in the Measurement of Workplace Incivility. In J. Greenberg (Ed.), Insidious workplace behavior (pp. 239-271). New York: Routledge.

Jiang, J. Y., Zhang, X., \& Tjosvold, D. (2013). Emotion regulation as a boundary condition of the relationship between team conflict and performance: A multilevel examination. Journal of Organizational Behavior, 34, 714-734. https://doi.org/10.1002/job.1834

John, O. P., \& Gross, J. J. (2004). Healthy and unhealthy emotion regulation: Personality processes, individual differences, and life span development. Journal of Personality, 72, 1301-1333. https://doi.org/10.1111/j.1467-6494.2004.00298.x

John, O. P., \& Gross, J. J. (2007). Individual differences in emotion regulation. In J. J. Gross (Ed.), Handbook of emotion regulation (pp. 351-372). New York: Guilford Press. 
John, O. P., Naumann, L. P., \& Soto, C. J. (2008). Paradigm shift to the integrative BigFive trait taxonomy: History, measurement, and conceptual issues. In O. P. John, R. W. Robins, \& L. Pervin (Eds.), Handbook of personality: Theory and research (pp. 114-158). New York, NY: Guilford Press.

Johnson, B. (2001). Toward a new classification of nonexperimental quantitative research. Educational Researcher, 30(2), 3-14. https://doi.org/10.3102/0013189X030002003

Johnson, D. R., \& Borden, L. A. (2012). Participants at your fingertipis: Using Amazon's Mechanical Turk to increase student-faculty collaborative research. Teaching of Psychology, 39, 245-251. https://doi.org/10.1177/0098628312456615

Johnson, R. B., Onwuegbuzie, A. J., \& Turner, L. A. (2007). Toward a definition of mixed methods research. Journal of Mixed Methods Research, 1, 112 - 133. doi: $10.1177 / 1558689806298224$.

Jöreskog, K. G. (1993). Testing structural equation models. In K. A. Bollen \& J. S. Long (Eds.), Testing structural equation models (pp. 294-316). Newbury Park, CA: Sage.

Jost, J. T., \& Thompson, E. P. (2000). Group-based dominance and opposition to equality as independent predictors of self-esteem, ethnocentrism, and social policy attitudes among African Americans and European Americans. Journal of Experimental Social Psychology, 36, 209-232. https://doi.org/10.1006/jesp.1999.1403

Kabat-Farr, D., \& Cortina, L. M. (2012). Selective incivility: Gender, race, and the discriminatory workplace. In S. Fox \& T. Lituchy (Eds.), Gender and the dysfunctional workplace (pp. 107-119). Northhampton, MA: Edward Elgar; https://doi.org/10.4337/9780857932600.00014

Kammeyer-Mueller, J., Wanberg, C., Rubenstein, A., \& Song, Z. (2013). Support, undermining, and newcomer socialization: Fitting in during the first 90 days. Academy of Management Journal, 56, 1104-1124. https://doi.org/10.5465/amj.2010.0791

Kashdan, T. B., Barrios, V., Forsyth, J. P., \& Steger, M. F. (2006). Experiential avoidance as a generalized psychological vulnerability: Comparisons with coping and emotion regulation strategies. Behaviour Research and Therapy, 44, 13011320. https://doi.org/10.1016/j.brat.2005.10.003

Katz, I., \& Hass, R. G. (1988). Racial ambivalence and American value conflict: Correlational and priming studies of dual cognitive structures. Journal of 
Personality and Social Psychology, 55, 893-905. https://doi.org/10.1037/00223514.55.6.893

Keith, M., \& Harms, P. (2016). Is Mechanical Turk the answer to our sampling woes? Industrial and Organizational Psychology: Perspectives on Science and Practice, 9, 162-169. https://doi.org/10.1017/iop.2015.130

Kemmelmeier, M., Danielson, C., \& Basten, J. (2005). What's in a grade? Academic success and political orientation. Personality and Social Psychology Bulletin, 31, 1386-1399. https://doi.org/10.1177/0146167205276085

Kenny, D. A., \& McCoach, D. B. (2003). Effect of the number of variables on measures of fit in structural equation modeling. Structural Equation Modeling, 10, 333351. http://dx.doi.org/10.1207/S15328007SEM1003 1

Kern, J. H., \& Grandey, A. A. (2009). Customer incivility as a social stressor: The role of race and racial identity for service employees. Journal of Occupational Health Psychology, 14, 46-57. https://doi.org/10.1037/a0012684

Kim, E., Bhave, D. P., \& Glomb, T. M. (2013). Emotion regulation in workgroups: The roles of demographic diversity and relational work context. Personnel Psychology, 66, 613-644. https://doi.org/10.1111/peps.12028

King, L. A., \& Emmons, R. A. (1990). Conflict over emotional expression: Psychological and physical correlates. Journal of Personality and Social Psychology, 58, 864877. Retrieved from http://dx.doi.org.ezproxy.fiu.edu/10.1037/00223514.58.5.864 https://doi.org/10.1037/0022-3514.58.5.864

Kleinpenning, G., \& Hagendoorn, L. (1993). Forms of racism and the cumulative dimension of ethnic attitudes. Social Psychology Quarterly, 56, 21-36. Retrieved from http://www.jstor.org/stable/2786643 https://doi.org/10.2307/2786643

Kline, R. B. (2005). Principles and practice of structural equation modeling (2nd ed.). New York, NY: Guilford Press.

Kline, R. B. (1998). Principles and practice of structural equation modeling. New York, NY: Guilford.

Koole, S. L. (2009). The psychology of emotion regulation: An integrative review. Cognition and Emotion, 23, 4-41. https://doi.org/10.1080/02699930802619031

Kteily, N., Bruneau, E., Waytz, A., \& Cotterill, S. (2015). The ascent of man: Theoretical and empirical evidence for blatant dehumanization. Journal of Personality and Social Psychology, 109, 901-931. https://doi.org/10.1037/pspp0000048 
Kteily, N. S., Ho, A. K., \& Sidanius, J. (2012). Hierarchy in the mind: The predictive power of social dominance orientation across social contexts and domains. Journal of Experimental Social Psychology, 48, 543-549. https://doi.org/10.1016/j.jesp.2011.11.007

Kteily, N. S., Sidanius, J., \& Levin, S. (2011). Social dominance orientation: Cause or 'mere effect'? Evidence for SDO as a causal predictor of prejudice and discrimination against ethnic and racial outgroups. Journal of Experimental Social Psychology, 47, 208-214. https://doi.org/10.1016/j.jesp.2010.09.009

Kugler, M. B., Cooper, J., \& Nosek, B. A. (2010). Group-based dominance and opposition to equality correspond to different psychological motives. Social Justice Research, 23, 117-155. https://doi.org/10.1007/s11211-010-0112-5

Kunkel, D., \& Davidson, D. (2014). Taking the good with the bad: Measuring civility and incivility. Journal of Organizational Culture, Communications \& Conflict, 18, 215-232. Retrieved from http://www.alliedacademies.org/public/journals/JournalDetails.aspx?jid=11

Küpper, B., Wolf, C., \& Zick, A. (2010). Social status and anti-immigrant attitudes in Europe: An examination from the perspective of social dominance theory. International Journal of Conflict and Violence, 4, 205-219. Retrieved from http://ezproxy.fiu.edu/login?url=https://search-proquestcom.ezproxy.fiu.edu/docview/863244501?accountid=10901

Kusy, M. E., \& Holloway, E. L. (2009). Toxic workplace! Managing toxic personalities and their systems of power. San Francisco: Jossey-Bass.

Lance, C. E., Butts, M., \& Michels, L. C. (2006). The sources of four commonly reported cutoff criteria: What did they really say? Organizational Research Methods, 9, 202-220. https://doi.org/10.1177/1094428105284919

Landers, R. N., \& Behrend, T. S. (2015). An inconvenient truth: Arbitrary distinctions between organizational, Mechanical Turk, and other convenience samples. Industrial and Organizational Psychology: Perspectives on Science and Practice, 8, 142-164. https://doi.org/10.1017/iop.2015.13

Lazarus, R. S., \& Alfert, E. (1964). Short-circuiting of threat by experimentally altering cognitive appraisal. Journal of Abnormal and Social Psychology, 69, 195-205. https://doi.org/10.1037/h0044635

Lee, J. J., \& Ok, C. (2012). Reducing burnout and enhancing job satisfaction: Critical role of hotel employees' emotional intelligence and emotional labor. International Journal of Hospitality Management, 31, 1101-1112. https://doi.org/10.1016/j.ijhm.2012.01.007 
Lee, R. T., \& Brotheridge, C. M. (2006). When prey turns predatory: Workplace bullying as a predictor of counteraggression/bullying, coping, and well-being. European Journal of Work and Organizational Psychology, 15, 352-377. https://doi.org/10.1080/13594320600636531

Leiter, M. P., Laschinger, H. K. S., Day, A., \& Oore, D. G. (2011). The impact of civility interventions on employee social behavior, distress, and attitudes. The Journal of Applied Psychology, 96, 1258-1274. https://doi.org/10.1037/a0024442

Leiter, M. P., Price, S. L., \& Spence Laschinger, H. K. (2010). Generational differences in distress, attitudes and incivility among nurses. Journal of Nursing Management, 18, 970-980. https://doi.org/10.1111/j.1365-2834.2010.01168.x

Lench, H. C., Flores, S. A., \& Bench, S. W. (2011). Discrete emotions predict changes in cognition, judgment, experience, behavior, and physiology: A meta-analysis of experimental emotion elicitations. Psychological Bulletin, 137, 834-855. https://doi.org/10.1037/a0024244

Lerner, J. S., Li, Y., Valdesolo, P., \& Kassam, K. S. (2015). Emotion and decision making. Annual Review of Psychology, 66, 799-823. https://doi.org/10.1146/annurev-psych-010213-115043

Lim, S., \& Cortina, L. M. (2005). Interpersonal mistreatment in the workplace: The interface and impact of general incivility and sexual harassment. The Journal of Applied Psychology, 90, 483-496. https://doi.org/10.1037/0021-9010.90.3.483

Lim, S., Cortina, L. M., \& Magley, V. J. (2008). Personal and workgroup incivility: Impact on work and health outcomes. The Journal of Applied Psychology, 93, 95107. https://doi.org/10.1037/0021-9010.93.1.95

Lim, S., \& Lee, A. (2011). Work and nonwork outcomes of workplace incivility: Does family support help? Journal of Occupational Health Psychology, 16, 95-111. https://doi.org/10.1037/a0021726

Lim, V. K., \& Teo, T. S. (2009). Mind your e-manners: Impact of cyber incivility on employees' work attitude and behavior. Information \& Management, 46, 419425. https://doi.org/10.1016/j.im.2009.06.006

Lischetzke, T., \& Eid, M. (2006). Why extraverts are happier than introverts: The role of mood regulation. Journal of Personality, 74, 1127-1161. https://doi.org/10.1111/j.1467-6494.2006.00405.x

Litman, L., Robinson, J., \& Abberbock, T. (2017). TurkPrime.com: A versatile crowdsourcing data acquisition platform for the behavioral sciences. Behavior Research Methods, 49, 433-442. https://doi.org/10.3758/s13428-016-0727-z 
Litman, L., Robinson, J., \& Rosenzweig, C. (2015). The relationship between motivation, monetary compensation, and data quality among US- and India-based workers on Mechanical Turk. Behavior Research Methods, 47, 519-528. https://doi.org/10.3758/s13428-014-0483-X

Liu, J., Kwong Kwan, H., Wu, L. Z., \& Wu, W. (2010). Abusive supervision and subordinate supervisor-directed deviance: The moderating role of traditional values and the mediating role of revenge cognitions. Journal of Occupational and Organizational Psychology, 83, 835-856. https://doi.org/10.1348/096317909X485216

Liu, W., Steve Chi, S. C., Friedman, R., \& Tsai, M. H. (2009). Explaining incivility in the workplace: The effects of personality and culture. Negotiation and Conflict Management Research, 2, 164-184. https://doi.org/10.1111/j.17504716.2009.00035.x

Liu, Y., Prati, L. M., Perrewé, P. L., \& Brymer, R. A. (2010). Individual differences in emotion regulation, emotional experiences at work, and work-related outcomes: A two-study investigation. Journal of Applied Social Psychology, 40, 1515-1538. https://doi.org/10.1111/j.1559-1816.2010.00627.x

Lorenzi-Cioldi, F. (1998). Group status and perceptions of homogeneity. European Review of Social Psychology, 9, 31-75. https://doi.org/10.1080/14792779843000045

Macho, S., \& Ledermann, T. (2011). Estimating, testing, and comparing specific effects in structural equation models: The phantom model approach. Psychological Methods, 16, 34-43. https://doi.org/10.1037/a0021763

MacKinnon, D. (2012). Introduction to statistical mediation analysis. NY: Taylor \& Francis.

Magee, J. C., \& Galinsky, A. D. (2008). 8 Social Hierarchy: The self-reinforcing nature of power and status. The Academy of Management Annals, 2(1), 351-398. https://doi.org/10.5465/19416520802211628

Martin, R. J., \& Hine, D. W. (2005). Development and validation of the uncivil workplace behavior questionnaire. Journal of Occupational Health Psychology, 10, 477-490. https://doi.org/10.1037/1076-8998.10.4.477

Maslach, C. (1982). Burnout: The cost of caring. Englewood Cliffs, NJ: Prentice-Hall.

Maslach, C., Schaufeli, W. B., \& Leiter, M. P. (2001). Job burnout. Annual Review of Psychology, 52, 397-422. https://doi.org/10.1146/annurev.psych.52.1.397 
Mason, W., \& Suri, S. (2012). Conducting behavioral research on Amazon's Mechanical Turk. Behavior Research Methods, 44, 1-23. https://doi.org/10.3758/s13428-0110124-6

Mason, W., \& Watts, D. J. (2010). Financial incentives and the performance of crowds. SIGKDD Explorations, 11, 100-108. https://doi.org/10.1145/1809400.1809422

Matthews, M., \& Levin, S. (2012). Testing a dual process model of prejudice: Assessment of group threat perceptions and emotions. Motivation and Emotion, 36, 564-574. https://doi.org/10.1007/s11031-012-9280-y

Mauss, I. B., Levenson, R. W., McCarter, L., Wilhelm, F. H., \& Gross, J. J. (2005). The tie that binds? Coherence among emotion experience, behavior, and physiology. Emotion (Washington, D.C.), 5, 175-190. Retrieved from http://dx.doi.org.ezproxy.fiu.edu/10.1037/1528-3542.5.2.175 https://doi.org/10.1037/1528-3542.5.2.175

Mawritz, M. B., Dust, S. B., \& Resick, C. J. (2014). Hostile climate, abusive supervision, and employee coping: Does conscientiousness matter? The Journal of Applied Psychology, 99, 737-747. Retrieved from http://dx.doi.org.ezproxy.fiu.edu/10.1037/a0035863 https://doi.org/10.1037/a0035863

McConahay, J. B. (1986). Modern racism, ambivalence, and the modern racism scale. In J. F. Dovidio \& S. L. Gaertner (Eds.), Prejudice, discrimination, and racism (pp. 91-125). Orlando, FL: Academic Press.

McRae, K., Ochsner, K. N., Mauss, I. B., Gabrieli, J. J. D., \& Gross, J. J. (2008). Gender Differences in Emotion Regulation: An fMRI Study of Cognitive Reappraisal. Group Processes \& Intergroup Relations : GPIR, 11, 143-162. https://doi.org/10.1177/1368430207088035

Meier, L. L., \& Gross, S. (2015). Episodes of incivility between subordinates and supervisors: Examining the role of self-control and time with an interactionrecord diary study. Journal of Organizational Behavior, 36, 1096-1113. https://doi.org/10.1002/job.2013

Meier, L. L., \& Semmer, N. K. (2012). Lack of reciprocity, narcissism, anger, and instigated workplace incivility: A moderated mediation model. European Journal of Work and Organizational Psychology, 22, 461-475. https://doi.org/10.1080/1359432X.2012.654605

Mesmer-Magnus, J., Glew, D. J., \& Viswesvaran, C. (2012). A meta-analysis of positive humor in the workplace. Journal of Managerial Psychology, 27, 155-190. https://doi.org/10.1108/02683941211199554 
Meyers, L. S., Gamst, G., \& Guarino, A. J. (2016). Applied multivariate research: Design and interpretation. Sage Publications.

Miller, N., Pedersen, W. C., Earleywine, M., \& Pollock, V. E. (2003). Artificial a theoretical model of triggered displaced aggression. Personality and Social Psychology Review, 7, 57-97. https://doi.org/10.1207/S15327957PSPR0701_5

Miner-Rubino, K., \& Cortina, L. M. (2004). Working in a context of hostility toward women: Implications for employees' well-being. Journal of Occupational Health Psychology, 9, 107-122. Retrieved from http://dx.doi.org.ezproxy.fiu.edu/10.1037/1076-8998.9.2.107 https://doi.org/10.1037/1076-8998.9.2.107

Miner, K. N., Diaz, I., Wooderson, R. L., McDonald, J. N., Smittick, A. L., \& Lomeli, L. C. (2018). A workplace incivility roadmap: Identifying theoretical speedbumps and alternative routes for future research. Journal of Occupational Health Psychology, 23, 320-337. https://doi.org/10.1037/ocp0000093

Mizzi, R. C., \& Rocco, T. S. (2013). Deconstructing dominance: Toward a reconceptualization of the relationship between collective and individual identities, globalization, and learning at work. Human Resource Development Review, 12, 364-382. https://doi.org/10.1177/1534484313477410

Montgomery, K., Kane, K., \& Vance, C. M. (2004). Accounting for differences in norms of respect a study of assessments of incivility through the lenses of race and gender. Group \& Organization Management, 29, 248-268. https://doi.org/10.1177/1059601103252105

Moore, J. E. (2000). One road to turnover: An examination of work exhaustion in technology professionals. Management Information Systems Quarterly, 24, 141168. Retrieved from http://www.jstor.org/stable/3250982 https://doi.org/10.2307/3250982

Mount, M. K., Barrick, M. R., \& Stewart, G. L. (1998). Five-factor model of personality and performance in jobs involving interpersonal interactions. Human Performance, 11, 145-165. https://doi.org/10.1080/08959285.1998.9668029

Navarrete, C. D., McDonald, M. M., Molina, L. E., \& Sidanius, J. (2010). Prejudice at the nexus of race and gender: An outgroup male target hypothesis. Journal of Personality and Social Psychology, 98, 933-945. Retrieved from http://dx.doi.org.ezproxy.fiu.edu/10.1037/a0017931 https://doi.org/10.1037/a0017931 
Nezlek, J. B., \& Kuppens, P. (2008). Regulating positive and negative emotions in daily life. Journal of Personality, 76, 561-580. https://doi.org/10.1111/j.14676494.2008.00496.x

Nunnally, J. C. (1978). Psychometric theory (2nd ed.). New York: McGraw-Hill.

Gilin Oore, D., Leblanc, D., Day, A., Leiter, M. P., Spence Laschinger, H. K., Price, S. L., \& Latimer, M. (2010). When respect deteriorates: Incivility as a moderator of the stressor-strain relationship among hospital workers. Journal of Nursing Management, 18, 878-888. https://doi.org/10.1111/j.1365-2834.2010.01139.x

Oppenheimer, D. M., Meyvis, T., \& Davidenko, N. (2009). Instructional manipulation checks: Detecting satisficing to increase statistical power. Journal of Experimental Social Psychology, 45, 867-872.

https://doi.org/10.1016/j.jesp.2009.03.009

O’Rourke, N., Psych, R., \& Hatcher, L. (2013). A step-by-step approach to using SAS for factor analysis and structural equation modeling. SAS Institute.

Osatuke, K., Moore, S. C., Ward, C., Dyrenforth, S. R., \& Belton, L. (2009). Civility, respect, engagement in the workforce (CREW) nationwide organization development intervention at Veterans Health Administration. The Journal of Applied Behavioral Science, 45, 384-410. https://doi.org/10.1177/0021886309335067

Paolacci, G., \& Chandler, J. (2014). Inside the Turk: Understanding Mechanical Turk as a participant pool. Current Directions in Psychological Science, 23, 184-188. https://doi.org/10.1177/0963721414531598

Paolacci, G., Chandler, J., \& Ipeirotis, P. G. (2010). Running experiments on Amazon Mechanical Turk. Judgment and Decision Making, 5, 411-419. Retrieved from https://ssrn.com/abstract $=1626226$

Parkins, I. S., Fishbein, H. D., \& Ritchey, P. N. (2006). The influence of personality on workplace bullying and discrimination. Journal of Applied Social Psychology, 36, 2554-2577. https://doi.org/10.1111/j.0021-9029.2006.00117.x

Pearson, C. M., Andersson, L. M., \& Porath, C. L. (2000). Assessing and attacking workplace incivility. Organizational Dynamics, 29, 123-137. https://doi.org/10.1016/S0090-2616(00)00019-X

Pearson, C. M., Andersson, L. M., \& Porath, C. L. (2005). Workplace incivility. In S. Fox \& P. E. Spector (Eds.), Counterproductive work behaviour: Investigations of actors and targets (pp. 177-200). Washington, DC: American Psychological Association. https://doi.org/10.1037/10893-008 
Pearson, C. M., \& Porath, C. L. (2005). On the nature, consequences and remedies of workplace incivility: No time for "nice"? Think again. The Academy of Management Executive, 19(1), 7-18. https://doi.org/10.5465/AME.2005.15841946

Pearson, C., \& Porath, C. (2009). The costs of bad behavior. How incivility is damaging your business and what to do about it. New York, NY: Penguin Books Ltd.

Pedhazur, E. J., \& Schmelkin, L. P. (2013). Measurement, design, and analysis: An integrated approach. New York: Psychology Press.

Podsakoff, P. M., MacKenzie, S. B., Lee, J. Y., \& Podsakoff, N. P. (2003). Common method biases in behavioral research: A critical review of the literature and recommended remedies. The Journal of Applied Psychology, 88, 879-903. https://doi.org/10.1037/0021-9010.88.5.879

Porath, C. L., \& Pearson, C. M. (2010). The cost of bad behavior. Organizational Dynamics, 39, 64-71. https://doi.org/10.1016/j.orgdyn.2009.10.006

Porath, C. L., \& Pearson, C. M. (2012). Emotional and behavioral responses to workplace incivility and the impact of hierarchical status. Journal of Applied Social Psychology, 42(S1), E326-E357. https://doi.org/10.1111/j.15591816.2012.01020.x

Porath, C., \& Pearson, C. (2013). The price of incivility. Harvard Business Review, 91(12), 114-121, 146. Retrieved from http://eds.a.ebscohost.com.ezproxy.fiu.edu/ehost/pdfviewer/pdfviewer?vid=1\&sid =f84796af-0edc-46a5-8b94-bc91e5391901\%40sessionmgr4007

Porath, C. L., Overbeck, J. R., \& Pearson, C. M. (2008). Picking up the gauntlet: How individuals respond to status challenges. Journal of Applied Social Psychology, 38, 1945-1980. http://dx.doi.org/10.1111/j .1559-1816.2008.00375.x

Power, J. L., Brotheridge, C. M., Blenkinsopp, J., Bowes-Sperry, L., Bozionelos, N., Buzády, Z., \& Nnedumm, A. U. O. (2013). Acceptability of workplace bullying: A comparative study on six continents. Journal of Business Research, 66, 374380. https://doi.org/10.1016/j.jbusres.2011.08.018

Pratto, F., \& Stewart, A. L. (2012). Group dominance and the half-blindness of privilege. The Journal of Social Issues, 68, 28-45. https://doi.org/10.1111/j.15404560.2011.01734.x

Pratto, F., Sidanius, J., \& Levin, S. (2006). Social dominance theory and the dynamics of intergroup relations: Taking stock and looking forward. European Review of Social Psychology, 17, 271-320. https://doi.org/10.1080/10463280601055772 
Pratto, F., Sidanius, J., Stallworth, L. M., \& Malle, B. F. (1994). Social dominance orientation: A personality variable predicting social and political attitudes. Journal of Personality and Social Psychology, 67, 741-763. Retrieved from http://dx.doi.org.ezproxy.fiu.edu/10.1037/0022-3514.67.4.741 https://doi.org/10.1037/0022-3514.67.4.741

Pratto, F., Stewart, A. L., \& Zeineddine, F. B. (2013). When inequality fails: Power, group dominance, and societal change. Journal of Social and Political Psychology, 1, 132-160. https://doi.org/10.5964/jspp.v1i1.97

Reich, T. C., \& Hershcovis, M. S. (2015). Observing workplace incivility. The Journal of Applied Psychology, 100, 203-215. https://doi.org/10.1037/a0036464

Reio, T. G., Jr. (2010). The threat of common method variance bias to theory building. Human Resource Development Review, 9, 405-411. https://doi.org/10.1177/1534484310380331

Reio, T. G., Jr., \& Ghosh, R. (2009). Antecedents and outcomes of workplace incivility: Implications for human resource development research and practice. Human Resource Development Quarterly, 20, 237-264. https://doi.org/10.1002/hrdq.20020

Reio, T. G., Jr., \& Sanders-Reio, J. (2011). Thinking about workplace engagement: Does supervisor and coworker incivility really matter? Advances in Developing Human Resources, 13, 462-478. https://doi.org/10.1177/1523422311430784

Reio, T. G., Jr., \& Trudel, J. (2013). Workplace incivility and conflict management style: Predicting job performance, organizational commitment, and turnover intent. International Journal of Adult Vocational Education and Technology, 4, 15-37. https://doi.org/10.4018/ijavet.2013100102

Reio, T.G., Jr., \& Werner, J. M. (2017). Publishing mixed methods research: Thoughts and recommendations concerning rigor. Human Resource Development Quarterly, 28, 439 - 449. doi: 10.1002/hrdq.21291

Reynolds, W. M. (1982). Development of reliable and valid short forms of the MarloweCrowne Social Desirability Scale. Journal of Clinical Psychology, 38, 119-125. https://doi.org/10.1002/1097-4679(198201)38:13.0.CO;2-I

Richardson, H. A., Simmering, M. J., \& Sturman, M. C. (2009). A tale of three perspectives: Examining post hoc statistical techniques for detection and correction of common method variance. Organizational Research Methods, 12, 762-800. https://doi.org/10.1177/1094428109332834 
Rind, B., \& Strohmetz, D. (1999). Effect on restaurant tipping of a helpful message written on the back of customers' checks. Journal of Applied Social Psychology, 29, 139-144. https://doi.org/10.1111/j.1559-1816.1999.tb01378.x

Rigdon, E. E., Schumacker, R. E., \& Wothke, W. (1998). A comparative review of interaction and nonlinear modeling. In R. E. Schumacker \& G. A. Marcoulides (Eds.), Interaction and Nonlinear Effects in Structural Equation Modeling (pp. 116). Mahwah, NJ: Lawrence Erlbaum Associates.

Roscigno, V. J., Lopez, S. H., \& Hodson, R. (2009). Supervisory bullying, status inequalities and organizational context. Social Forces, 87, 1561-1589. https://doi.org/10.1353/sof.0.0178

Rose, J. S., Chassin, L., Presson, C. C., \& Sherman, S. J. (2000). Contrasts in multiple mediator models. In Multivariate applications in substance use research (pp. 155-174). Psychology Press. https://doi.org/10.4324/9781410604217

Rouse, S. (2015) A reliability analysis of Mechanical Turk data. Computers in Human Behavior. 43, 304-307. doi/10.1016/j.chb.2014.11.004.

Sakurai, K., \& Jex, S. M. (2012). Coworker incivility and incivility targets' work effort and counterproductive work behaviors: The moderating role of supervisor social support. Journal of Occupational Health Psychology, 17, 150-161. https://doi.org/10.1037/a0027350

Schilpzand, P., De Pater, I. E., \& Erez, A. (2016). Workplace incivility: A review of the literature and agenda for future research. Journal of Organizational Behavior, 37(S1), S57-S88. https://doi.org/10.1002/job.1976

Schmitt, M. T., \& Wirth, J. H. (2009). Evidence that gender differences in social dominance orientation result from gendered self-stereotyping and groupinterested responses to patriarchy. Psychology of Women Quarterly, 33, 429-436. https://doi.org/10.1111/j.1471-6402.2009.01520.x

Schreiber, J. B., Nora, A., Stage, F. K., Barlow, E. A., \& King, J. (2006). Reporting structural equation modeling and confirmatory factor analysis results: A review. The Journal of Educational Research, 99, 323-338. https://doi.org/10.3200/JOER.99.6.323-338

Schumacher, R. E., \& Lomax, R. G. (2010). A beginner's guide to structural equation modeling. Mahwah, New Jersey: Lawrence Erlbaum Associates.

Schwartz, S. H. (1992). Universals in the content and structure of values: Theoretical advances and empirical tests in 20 countries. In Vol. 25, pp. 1-65). Advances in 
experimental social psychology Academic Press. https://doi.org/10.1016/S00652601(08)60281-6

Schwartz, S. H. (1999). A theory of cultural values and some implications for work. Applied Psychology, 48, 23-47. https://doi.org/10.1111/j.14640597.1999.tb00047.x

Schwartz, S. H. (2012). An overview of the Schwartz theory of basic values. Online Readings in Psychology and Culture, 2(1). https://doi.org/10.9707/23070919.1116

Scott, K. D., Restubog, S. L. D., \& Zagenczyk, T. J. (2013). A social exchange-based model of the antecedents of workplace exclusion. Journal of Applied Psychology, 98, 37-48. doi:1037/a0030135

Shao, P., Resick, C. J., \& Hargis, M. B. (2011). Helping and harming others in the workplace: The roles of personal values and abusive supervision. Human Relations, 64, 1051-1078. https://doi.org/10.1177/0018726711399940

Shapiro, D., Chandler, J., \& Mueller, P. (2013). Using Mechanical Turk to study clinical populations. Clinical Psychological Science, 1, 213-220. https://doi.org/10.1177/2167702612469015

Shuck, B., Collins, J. C., Rocco, T. S., \& Diaz, R. (2016). Deconstructing the privilege and power of employee engagement: Issues of inequality for management and human resource development. Human Resource Development Review, 15, 208229. https://doi.org/10.1177/1534484316643904

Sibley, C. G., \& Duckitt, J. (2008). Personality and prejudice: A meta-analysis and theoretical review. Personality and Social Psychology Review, 12, 248-279. https://doi.org/10.1177/1088868308319226

Sibley, C. G., Wilson, M. S., \& Duckitt, J. (2007). Antecedents of men's hostile and benevolent sexism: The dual roles of social dominance orientation and right-wing authoritarianism. Personality and Social Psychology Bulletin, 33, 160-172. https://doi.org/10.1177/0146167206294745

Sidanius, J., \& Pratto, F. (1993). The inevitability of oppression and the dynamics of social dominance. In P. Sniderman, P. E. Tetlock, \& E. G. Carmines (Eds.), Prejudice, politics, and the American dilemma (pp. 173-211). Stanford, CA: Stanford University Press.

Sidanius, J., \& Pratto, F. (1999). Social dominance: An intergroup theory of social hierarchy and oppression. New York: Cambridge University Press. https://doi.org/10.1017/CBO9781139175043 
Sidanius, J., \& Pratto, F. (2012). Social dominance theory. In P. A. M. Van Lange, A. W. Kruglanski, \& E. T. Higgins (Eds.), Handbook of theories of social psychology (Vol. 2, pp. 418-438). Thousand Oaks, CA: Sage. https://doi.org/10.4135/9781446249222.n47

Sidanius, J., Pratto, F., \& Mitchell, M. (1994). In-group identification, social dominance orientation, and differential intergroup social allocation. The Journal of Social Psychology, 134, 151-167. https://doi.org/10.1080/00224545.1994.9711378

Sidanius, J., Pratto, F., Van Laar, C., \& Levin, S. (2004). Social dominance theory: Its agenda and method. Political Psychology, 25, 845-880.

https://doi.org/10.1111/j.1467-9221.2004.00401.x

Siomkos, G. J., Rao, S. S., \& Narayanan, S. (2001). The influence of positive and negative affectivity on attitude change toward organizations. Journal of Business and Psychology, 16, 151-161. https://doi.org/10.1023/A:1007800124297

Skarlicki, D. P., \& Folger, R. (1997). Retaliation in the workplace: The roles of distributive, procedural and interactional justice. The Journal of Applied Psychology, 82, 434-443. Retrieved from http://dx.doi.org.ezproxy.fiu.edu/10.1037/0021-9010.82.3.434 https://doi.org/10.1037/0021-9010.82.3.434

Sliter, K. A., Sliter, M. T., Withrow, S. A., \& Jex, S. M. (2012a). Employee adiposity and incivility: Establishing a link and identifying demographic moderators and negative consequences. Journal of Occupational Health Psychology, 17, 409424. https://doi.org/10.1037/a0029862

Sliter, M., Jex, S., Wolford, K., \& McInnerney, J. (2010). How rude! Emotional labor as a mediator between customer incivility and employee outcomes. Journal of Occupational Health Psychology, 15, 468-481. https://doi.org/10.1037/a0020723

Sliter, M. T., Sliter, K. A., \& Jex, S. (2012b). The employee as a punching bag: The effect of multiple sources of incivility on employee withdrawal behavior and sales performance. Journal of Organizational Behavior, 33, 121-139. https://doi.org/10.1002/job.767

Snellman, A., Ekehammar, B., \& Akrami, N. (2009). The role of gender identification in social dominance orientation: Mediating or moderating the effect of sex? Journal of Applied Social Psychology, 39, 999-1012. https://doi.org/10.1111/j.15591816.2009.00469.x

Spector, P. E. (2006). Method variance in organizational research: Truth or urban legend? Organizational Research Methods, 9, 221-232. https://doi.org/10.1177/1094428105284955 
Spence Laschinger, H. K., Leiter, M., Day, A., \& Gilin, D. (2009). Workplace empowerment, incivility, and burnout: Impact on staff nurse recruitment and retention outcomes. Journal of Nursing Management, 17, 302-311. https://doi.org/10.1111/j.1365-2834.2009.00999.x

Spence Laschinger, H. K., Leiter, M. P., Day, A., Gilin-Oore, D., \& Mackinnon, S. P. (2012). Building empowering work environments that foster civility and organizational trust: Testing an intervention. Nursing Research, 61, 316-325. https://doi.org/10.1097/NNR.0b013e318265a58d

Sprouse, J. (2011). A validation of Amazon Mechanical Turk for the collection of acceptability judgments in linguistic theory. Behavior Research Methods, 43, 155-167. https://doi.org/10.3758/s13428-010-0039-7

Stefanucci, J. K., Gagnon, K. T., \& Lessard, D. A. (2011). Follow your heart: Emotion adaptively influences perception. Social and Personality Psychology Compass, 5, 296-308. https://doi.org/10.1111/j.1751-9004.2011.00352.x

Stevens, J. P. (2009). Applied multivariate statistics for the social sciences (5th ed.). Mahwah, NJ: Routledge Academic.

Sugiura, H., Mifune, N., Tsuboi, S., \& Yokota, K. (2017). Gender differences in intergroup conflict: The effect of outgroup threat priming on social dominance orientation. Personality and Individual Differences, 104, 262-265. https://doi.org/10.1016/j.paid.2016.08.013

Suri, S., \& Watts, D. J. (2011). Cooperation and contagion in web-based, networked public goods experiments. PLoS One, 6(3), e16836. https://doi.org/10.1371/journal.pone.0016836

Swim, J. K., Mallett, R., Russo-Devosa, Y., \& Stangor, C. (2005). Judgments of sexism: A comparison of the subtlety of sexism measures and sources of variability in judgments of sexism. Psychology of Women Quarterly, 29, 406-411. https://doi.org/10.1111/j.1471-6402.2005.00240.x

Taylor, S. G., \& Kluemper, D. H. (2012). Linking perceptions of role stress and incivility to workplace aggression: The moderating role of personality. Journal of Occupational Health Psychology, 17, 316-329. https://doi.org/10.1037/a0028211

Tepper, B. J. (2000). Consequences of abusive supervision. Academy of Management Journal, 43, 178-190. https://doi.org/10.2307/1556375

Tett, R. P., Jackson, D. N., \& Rothstein, M. (1991). Personality measures as predictors of job performance: A meta-analytic review. Personnel Psychology, 44, 703-742. https://doi.org/10.1111/j.1744-6570.1991.tb00696.x 
Tinkler, S., \& Woods, J. (2013). The readability of principles of macroeconomics textbooks. The Journal of Economic Education, 44, 178-191. https://doi.org/10.1080/00220485.2013.770345

Torkelson, E., Holm, K., Bäckström, M., \& Schad, E. (2016). Factors contributing to the perpetration of workplace incivility: The importance of organizational aspects and experiencing incivility from others. Work and Stress, 30, 115-131. https://doi.org/10.1080/02678373.2016.1175524

Totterdell, P., Hershcovis, M. S., Niven, K., Reich, T. C., \& Stride, C. (2012). Can employees be emotionally drained by witnessing unpleasant interactions between coworkers? A diary study of induced emotion regulation. Work \& Stress, 26, 112 129. doi:10.1080/02678373.2012.681153

Troy, A. S., Wilhelm, F. H., Shallcross, A. J., \& Mauss, I. B. (2010). Seeing the silver lining: Cognitive reappraisal ability moderates the relationship between stress and depressive symptoms. Emotion (Washington, D.C.), 10, 783-795. https://doi.org/10.1037/a0020262

Trudel, J., \& Reio, T. G., Jr. (2011). Managing workplace incivility: The role of conflict management styles-Antecedent or antidote? Human Resource Development Quarterly, 22, 395-423. doi:10 .1002/hrdq.20081

Trudel-Fitzgerald, C., Qureshi, F., Appleton, A. A., \& Kubzansky, L. D. (2017). A healthy mix of emotions: Underlying biological pathways linking emotions to physical health. Current Opinion in Behavioral Sciences, 15, 16-21. https://doi.org/10.1016/j.cobeha.2017.05.003

Turner, A. M., Kirchhoff, K., \& Capurro, D. (2012). Using crowdsourcing technology for testing multilingual public health promotion materials. Journal of Medical Internet Research, 14(3), e79. Retrieved from http://doi.org.ezproxy.fiu.edu/10.2196/jmir.2063 https://doi.org/10.2196/jmir.2063

Van Dijk, P. A., \& Brown, A. K. (2006). Emotional labour and negative job outcomes: An evaluation of the mediating role of emotional dissonance. Journal of Management \& Organization, 12, 101-115. https://doi.org/10.5172/jmo.2006.12.2.101

Van Hiel, A., Duriez, B., \& Kossowska, M. (2006). The presence of left-wing authoritarianism in Western Europe and its relationship with conservative ideology. Political Psychology, 27, 769-793. https://doi.org/10.1111/j.14679221.2006.00532.x 
Van Jaarsveld, D. D., Walker, D. D., \& Skarlicki, D. P. (2010). The role of job demands and emotional exhaustion in the relationship between customer and employee incivility. Journal of Management, 36, 1486-1504. https://doi.org/10.1177/0149206310368998

Vardi, Y., \& Weitz, E. (2004). Misbehavior in organizations: Theory, research and management. Mahwah, New Jersey: Lawrence Erlbaum Associates.

Vargas-Salfate, S., Paez, D., Liu, J. H., Pratto, F., \& Gil de Zúñiga, H. (2018). A comparison of social dominance theory and system justification: The role of social status in 19 nations. Personality and Social Psychology Bulletin, 44, 10601076. https://doi.org/10.1177/0146167218757455

Vickers, M. H. (2006). Writing what's relevant: Workplace incivility in public administration-A wolf in sheep's clothing. Administrative Theory \& Praxis, 28, 69-88. https://doi.org/10.1080/10841806.2006.11029525

Walker, D. D., van Jaarsveld, D. D., \& Skarlicki, D. P. (2014). Exploring the effects of individual customer incivility encounters on employee incivility: The moderating roles of entity (in)civility and negative affectivity. The Journal of Applied Psychology, 99, 151-161. Retrieved from http://dx.doi.org.ezproxy.fiu.edu/10.1037/a0034350 https://doi.org/10.1037/a0034350

Walsh, B. M., Lee, J. J., Jensen, J. M., McGonagle, A. K., \& Samnani, A. K. (2018). Positive leader behaviors and workplace incivility: The mediating role of perceived norms for respect. Journal of Business and Psychology, 33, 495-508. https://doi.org/10.1007/s10869-017-9505-x

Walsh, B. M., Magley, V. J., Reeves, D. W., Davies-Schrils, K. A., Marmet, M. D., \& Gallus, J. A. (2012). Assessing workgroup norms for civility: The development of the Civility Norms Questionnaire-Brief. Journal of Business and Psychology, 27, 407-420. https://doi.org/10.1007/s10869-011-9251-4

Watson, D., Clark, L. A., \& Tellegen, A. (1988). Development and validation of brief measures of positive and negative affect: The PANAS scales. Journal of Personality and Social Psychology, 54, 1063-1070. Retrieved from http://dx.doi.org.ezproxy.fiu.edu/10.1037/0022-3514.54.6.1063 https://doi.org/10.1037/0022-3514.54.6.1063

Webb, T. L., Miles, E., \& Sheeran, P. (2012). Dealing with feeling: A meta-analysis of the effectiveness of strategies derived from the process model of emotion regulation. Psychological Bulletin, 138, 775-808. https://doi.org/10.1037/a0027600 
Westfall, P. H., \& Henning, K. S. S. (2013). Texts in statistical science: Understanding advanced statistical methods. Boca Raton, FL: Taylor \& Francis.

Whitla, P. (2009). Crowdsourcing and its application in marketing activities. Contemporary Management Research, 5, 15-28. https://doi.org/10.7903/cmr.1145

Williams, L. J., \& Anderson, S. E. (1991). Job satisfaction and organizational commitment as predictors of organizational citizenship and in-role behaviors. Journal of Management, 17, 601-617. https://doi.org/10.1177/014920639101700305

Williams, D. P., Cash, C., Rankin, C., Bernardi, A., Koenig, J., \& Thayer, J. F. (2015). Resting heart rate variability predicts self-reported difficulties in emotion regulation: A focus on different facets of emotion regulation. Frontiers in Psychology, 6, 261. https://doi.org/10.3389/fpsyg.2015.00261

Wilson, N. L., \& Holmvall, C. M. (2013). The development and validation of the Incivility from Customers Scale. Journal of Occupational Health Psychology, 18, 310-326. Retrieved from http://dx.doi.org.ezproxy.fiu.edu/10.1037/a0032753 https://doi.org/10.1037/a0032753

Wiltink, J., Glaesmer, H., Canterino, M., Wölfling, K., Knebel, A., Kessler, H., Beutler, M. E. (2011). Regulation of emotions in the community: Suppression and reappraisal strategies and its psychometric properties. GMS Psycho-SocialMedicine, 8, Article Doc9. doi:10.3205/psm000078

Wright, K. B. (2005). Researching Internet-based populations: Advantages and disadvantages of online survey research, online questionnaire authoring software packages, and web survey services. Journal of Computer-Mediated Communication, 10, JCMC1034. https://doi.org/10.1111/j.10836101.2005.tb00259.x

Wu, L. Z., Zhang, H., Chiu, R. K., Kwan, H. K., \& He, X. (2014). Hostile attribution bias and negative reciprocity beliefs exacerbate incivility's effects on interpersonal deviance. Journal of Business Ethics, 120, 189-199. https://doi.org/10.1007/s10551-013-1658-6

Xin, Z., \& Chi, L. (2010). Wording effect leads to a controversy over the construct of the social dominance orientation scale. The Journal of Psychology, 144, 473-488. https://doi.org/10.1080/00223980.2010.496672

Zakrisson, I. (2008). Gender differences in social dominance orientation: Gender invariance may be situation invariance. Sex Roles, 59, 254-263. https://doi.org/10.1007/s11199-008-9445-z 
Zapf, D., \& Holz, M. (2006). On the positive and negative effects of emotion work in organizations. European Journal of Work and Organizational Psychology, 15, 128. https://doi.org/10.1080/13594320500412199 


\section{Appendix}

\begin{tabular}{|c|c|c|c|c|c|c|}
\hline & Social Domi & ance Ori & tion Sca & $\mathrm{SDO}_{7} ; \mathrm{H}$ & et al., 2015) & \\
\hline $\begin{array}{l}\text { Instruction } \\
\text { number fro } \\
\text { generally } b\end{array}$ & $\begin{array}{l}\text { Show how } \\
\text { n } 1 \text { to } 7 \text { on th } \\
\text { st. }\end{array}$ & $\begin{array}{l}\text { ich you } \mathrm{f} \\
\text { scale bel }\end{array}$ & $\begin{array}{l}\text { or oppo } \\
\text { You can }\end{array}$ & $\begin{array}{l}\text { ch idea } \\
\text { k quickl }\end{array}$ & $\begin{array}{l}\text { ow by selec } \\
\text { our first fe }\end{array}$ & $\begin{array}{l}\mathrm{ng} \text { a } \\
\text { ing is }\end{array}$ \\
\hline $\begin{array}{c}1 \\
\text { Strongly } \\
\text { Oppose }\end{array}$ & $\begin{array}{c}2 \\
\text { Somewhat } \\
\text { Oppose }\end{array}$ & $\begin{array}{c}3 \\
\text { Slightly } \\
\text { Oppose }\end{array}$ & $\begin{array}{c}4 \\
\text { Neutral }\end{array}$ & $\begin{array}{c}5 \\
\text { Slightly } \\
\text { Favor }\end{array}$ & $\begin{array}{c}6 \\
\text { Somewhat } \\
\text { Favor }\end{array}$ & $\begin{array}{c}7 \\
\text { Strongly } \\
\text { Favor }\end{array}$ \\
\hline 1. Sor & groups of $\mathrm{p}$ & ple mus & ept in $\mathrm{t}$ & place. & & \\
\hline $\begin{array}{l}\text { 2. It's } \\
\text { at t }\end{array}$ & $\begin{array}{l}\text { robably a go } \\
\text { e bottom. }\end{array}$ & thing $\mathrm{t}$ & rtain $g$ & are at $\mathrm{tl}$ & $\mathrm{p}$ and othe & groups are \\
\hline $\begin{array}{l}\text { 3. An } \\
\text { bot }\end{array}$ & $\begin{array}{l}\text { deal society } 1 \\
\mathrm{~m} \text {. }\end{array}$ & uires sol & roups to & on top an & thers to be & the \\
\hline 4. Sor & e groups of $p$ & ople are s & ly inferic & other grc & & \\
\hline 5. Grc & ips at the bot & $\mathrm{m}$ are jus & deservin & groups a & he top. & \\
\hline 6. $\mathrm{No}$ & ne group sho & ld domin & in society & & & \\
\hline 7. Grc & ips at the bot & m should & thave to & in their $\mathrm{p}$ & & \\
\hline 8. Grc & p dominance & is a poor 1 & ciple. & & & \\
\hline 9. $\mathrm{We}$ & hould not pu & $\mathrm{h}$ for grou & quality. & & & \\
\hline 10. We & houldn't try & guarante & lat every & has the & me quality & flife. \\
\hline 11. It is & unjust to try & make gr & s equal. & & & \\
\hline 12. Gro & ip equality sh & uld not b & Ir primar & & & \\
\hline 13. We & hould work & give all & ips an eq & chance to & cceed. & \\
\hline 14. $\mathrm{We}$ & hould do wh & $\mathrm{t}$ we can $\mathrm{t}$ & qualize ce & tions for & ferent group & \\
\hline $\begin{array}{l}\text { 15. No } \\
\text { hav }\end{array}$ & $\begin{array}{l}\text { natter how m } \\
\text { the same ch }\end{array}$ & $\begin{array}{l}\text { ch effort } \\
\text { ice in life }\end{array}$ & kes, we o & it to strive & ensure that & 11 groups \\
\hline 16. Grc & ip equality sh & uld be ou & eal. & & & \\
\hline $\begin{array}{l}* \text { SDO-D It } \\
* \text { SDO-E It } \\
* \text { Reverse } C\end{array}$ & $\begin{array}{l}\mathrm{ms}: 1 \text { to } 8 \\
\mathrm{~ms}: 9 \text { to } 10 \\
\text { de Items: } 5\end{array}$ & 3 and 13 & & & & \\
\hline
\end{tabular}




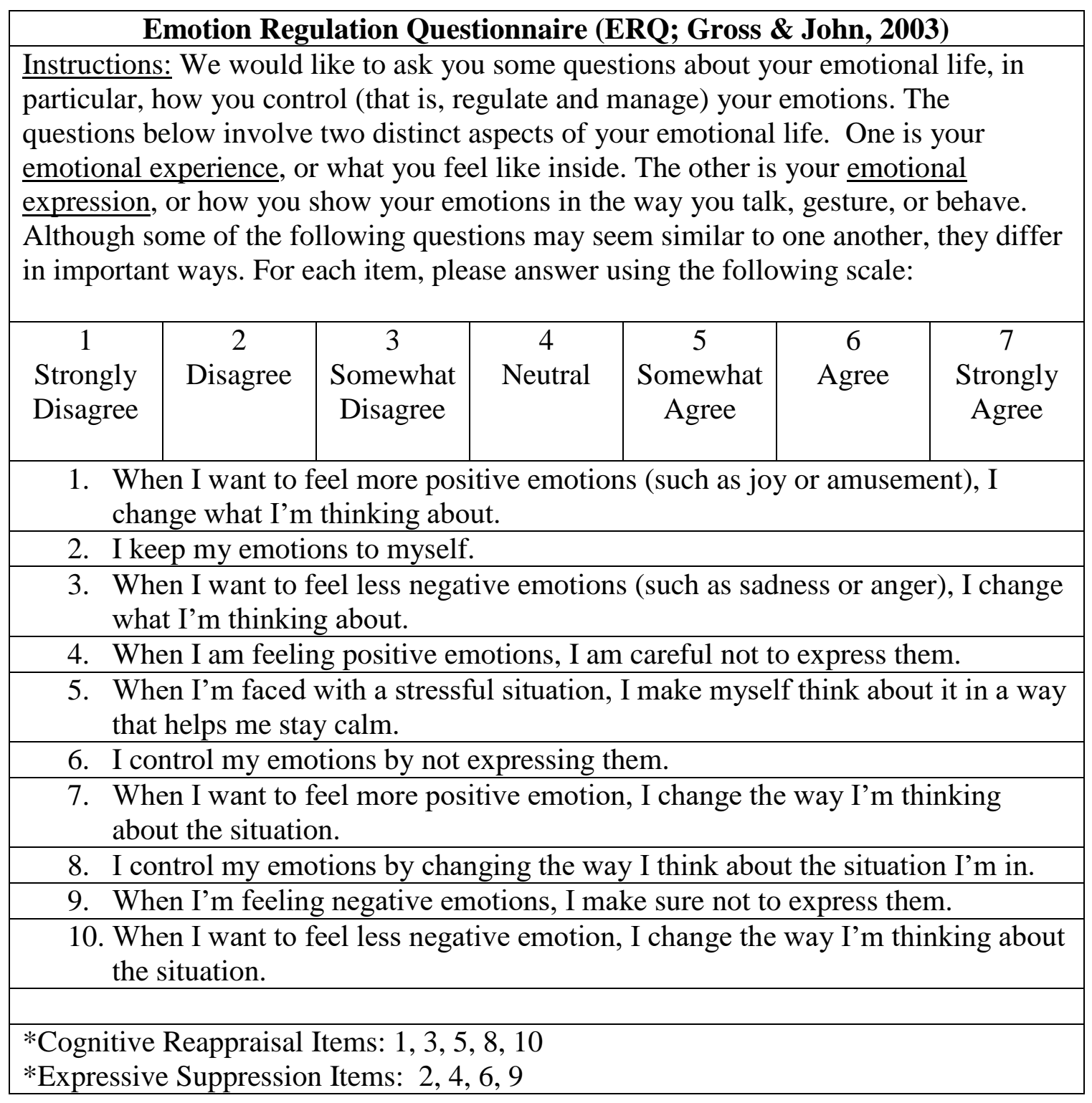




\section{(Adapted) Uncivil Workplace Behavior Questionnaire -Instigated (UWBQ-I; Gray et al., 2017)}

Instructions: Please indicate how often in the past year you have engaged in each of the following activities toward a co-worker while at work.

\begin{tabular}{|c|c|c|c|}
\hline $\begin{array}{c}1 \\
\text { Hardly Ever } \\
\text { (once every few } \\
\text { months or so) }\end{array}$ & $\begin{array}{c}2 \\
\text { Rarely } \\
\text { (about once a } \\
\text { month) }\end{array}$ & $\begin{array}{c}\underline{3} \\
\text { Sometimes } \\
\text { (at least once a } \\
\text { week) }\end{array}$ & $\begin{array}{c}\underline{4} \\
\text { Frequently } \\
\text { (at least once a day) }\end{array}$ \\
\hline \multicolumn{4}{|c|}{ 1. Raised your voice while speaking to a co-worker. } \\
\hline \multicolumn{4}{|c|}{ 2. Used an inappropriate tone when speaking to a co-worker. } \\
\hline \multicolumn{4}{|c|}{ 3. Spoke to a co-worker in an aggressive tone of voice. } \\
\hline \multicolumn{4}{|c|}{ 4. Rolled your eyes at a co-worker. } \\
\hline \multicolumn{4}{|c|}{ 5. Took stationary from a co-workers desk without later returning it. } \\
\hline \multicolumn{4}{|c|}{ 6. Took items from a co-workers desk without prior permission. } \\
\hline \multicolumn{4}{|c|}{ 7. Interrupted a co-worker while they were speaking on the telephone. } \\
\hline \multicolumn{4}{|c|}{ 8. Read communications addressed to a co-worker, such as emails or faxes. } \\
\hline \multicolumn{4}{|c|}{ 9. Opened a co-workers desk drawers without permission. } \\
\hline \multicolumn{4}{|c|}{$\begin{array}{l}\text { 10. Did not consult a co-worker in reference to a decisions that should have } \\
\text { involved them. }\end{array}$} \\
\hline \multicolumn{4}{|c|}{$\begin{array}{l}\text { 11. Gave unreasonably short notice when cancelling or scheduling events a co- } \\
\text { worker was required to be present for. }\end{array}$} \\
\hline \multicolumn{4}{|c|}{$\begin{array}{l}\text { 12. Failed to inform a co-worker of a meeting they should have been informed } \\
\text { about. }\end{array}$} \\
\hline \multicolumn{4}{|c|}{$\begin{array}{l}\text { 13. Avoided consulting with a co-worker when you would normally be expected to } \\
\text { do so. }\end{array}$} \\
\hline \multicolumn{4}{|c|}{$\begin{array}{l}\text { 14. Was excessively slow in returning a co-workers phone message or email } \\
\text { without good reason for the delay. }\end{array}$} \\
\hline \multicolumn{4}{|c|}{$\begin{array}{l}\text { 15. Intentionally failed to pass on information which your co-worker should have } \\
\text { been made aware of. }\end{array}$} \\
\hline \multicolumn{4}{|c|}{$\begin{array}{l}\text { 16. Were unreasonably slow in seeing to matters on which your co-worker was } \\
\text { reliant on you for, without good reason. }\end{array}$} \\
\hline \multicolumn{4}{|c|}{ 17. Publicly discussed a co-workers confidential personal information. } \\
\hline \multicolumn{4}{|c|}{ 18. Made snide remarks about a co-worker. } \\
\hline \multicolumn{4}{|c|}{ 19. Talked about a co-worker behind his/her back. } \\
\hline \multicolumn{4}{|c|}{ 20. Gossiped behind a co-workers back. } \\
\hline $\begin{array}{l}\text { Hostility Items } \\
\text { Privacy Invasic }\end{array}$ & 5 to 9 & $\begin{array}{l}\text { *Exclusionary B } \\
\text { *Gossiping Item }\end{array}$ & $\begin{array}{l}\text { ior Items: } 10 \text { to } 1 \\
\text { to } 20\end{array}$ \\
\hline
\end{tabular}




\begin{tabular}{|c|c|c|c|c|c|c|}
\hline \multicolumn{7}{|c|}{ Civility Norms Questionnaire - Brief (CNQ-B; Walsh et al., 2012) } \\
\hline \multicolumn{7}{|c|}{$\begin{array}{l}\text { Instructions: Please indicate how much you disagree or agree with the following } \\
\text { statements: }\end{array}$} \\
\hline $\begin{array}{c}1 \\
\text { Strongly } \\
\text { Disagree }\end{array}$ & $\begin{array}{c}2 \\
\text { Disagree }\end{array}$ & $\begin{array}{c}3 \\
\text { Somewhat } \\
\text { Disagree }\end{array}$ & $\begin{array}{c}4 \\
\text { Neutral }\end{array}$ & $\begin{array}{c}5 \\
\text { Somewhat } \\
\text { Agree }\end{array}$ & $\begin{array}{c}6 \\
\text { Agree }\end{array}$ & $\begin{array}{c}7 \\
\text { Strongly } \\
\text { Agree }\end{array}$ \\
\hline \multicolumn{7}{|c|}{ 1. Rude behavior is not accepted by your co-workers. } \\
\hline \multicolumn{7}{|c|}{ 2. Angry outbursts are not tolerated by anyone in your unit/workgroup. } \\
\hline \multicolumn{7}{|c|}{ 3. Respectful treatment is the norm in your unit/workplace. } \\
\hline \multicolumn{7}{|c|}{$\begin{array}{l}\text { 4. Your co-workers make sure everyone in your unit/workplace is treated with } \\
\text { respect. }\end{array}$} \\
\hline
\end{tabular}

\begin{tabular}{|c|c|c|}
\hline \multicolumn{3}{|c|}{ Marlow-Crowne Social Desirability Scale- Short Form C (Reynolds, 1982) } \\
\hline \multicolumn{3}{|c|}{$\begin{array}{l}\text { Instructions: Listed below are a number of statements concerning personal attitudes } \\
\text { and traits. Reach each item and decide whether the statement is true or false as it } \\
\text { pertains to you personally. }\end{array}$} \\
\hline & TRUE & FALSE \\
\hline $\begin{array}{l}\text { 1. It is sometimes hard for me to go on with my work if I'm not } \\
\text { encouraged. }\end{array}$ & & \\
\hline 2. I sometimes feel resentful when I don't bet my way. & & \\
\hline $\begin{array}{l}\text { 3. On a few occasions, I have given up doing something } \\
\text { because I thought too little of my ability. }\end{array}$ & & \\
\hline $\begin{array}{l}\text { 4. There have been times when I felt like rebelling against } \\
\text { people in authority even though I knew they were right. }\end{array}$ & & \\
\hline 5. No matter who I'm talking to, I'm always a good listener. & & \\
\hline $\begin{array}{l}\text { 6. There have been occasions when I took advantage of } \\
\text { someone. }\end{array}$ & & \\
\hline 7. I'm always willing to admit it when I make a mistake. & & \\
\hline 8. I sometimes try to get even rather than forgive and forget. & & \\
\hline 9. I am always courteous, even to people who are disagreeable. & & \\
\hline $\begin{array}{l}\text { 10. I have never been irked when people expressed ideas very } \\
\text { different from my own. }\end{array}$ & & \\
\hline $\begin{array}{l}\text { 11. There have been times when I was quite jealous of the good } \\
\text { fortune of others. }\end{array}$ & & \\
\hline 12. I am sometimes irritated by people who ask favors of me. & & \\
\hline $\begin{array}{l}\text { 13. I have never deliberately said something that hurt someone's } \\
\text { feelings. }\end{array}$ & & \\
\hline
\end{tabular}




\section{Demographic Questionnaire}

1. What is your age?

2. What is your gender?
a) Female
b) Male
c) Non-binary/third gender

3. How would you describe yourself?
a) American Indian
b) Alaska Native
c) Asian
d) Black
e) African American
f) Native American
g) Pacific Islander
h) Caucasian/White
i) Hispanic/Latino

4. In what country were you born?

5. What is the highest degree or level of school you have completed?
a) Less than a high school diploma
b) High school degree or equivalent (e.g. GED)
c) Some college, no degree
d) Associate degree (e.g. AA, AS)
e) Bachelor's degree (e.g. BA, BS)
f) Master's degree (e.g. MA, MS)
g) Professional degree (e.g. MD, DDS, DVM)
h) Doctorate (e.g. $\mathrm{PhD}, \mathrm{EdD})$

6. What is your political affiliation?
a) Democrat
b) Republican
c) Independent
d) Rather not say 
7. In what industry are you currently employed in?
a) Healthcare
b) Banking
c) Education
d) Manufacturing
e) Construction
f) Retail
g) Transportation
h) Arts and Entertainment
i) Real Estate
j) Accommodation and Food Service
k) Legal Services
1) Armed Forces
m) Federal Government

8. Roughly, how many people are employed at the place where you work?
a). $1-9$
b). $10-24$
c). $25-99$
d). $100-249$
e). $250+$

8. Does your current work require you to supervise the work of other employees?
a) Yes
b) No 
VITA

FABIANA BRUNETTA

Place of Birth Prodenone, Italy

2007

Associate of Arts in Psychology

Broward College, Hollywood, FL

2008

2009

Background Screening Coordinator

ChildNet, Plantation, FL

Bachelor of Arts in Psychology

Florida International University, Miami, FL

2011

Master of Science in Adult Education

Florida International University, Miami, FL

2011

Graduate Certificate, Conflict Resolution \& Consensus

Building

Florida International University, Miami, FL

2011

Graduate Research Assistant

Florida International University, Miami, FL

2015 Research and Evaluation Manager

Children's Services Council of Broward County, Sunrise, FL.

2016 - Present Research Consultant

Cuba Research Consultants Inc., Pembroke Pines, FL 


\section{PUBLICATIONS}

Grenier, G., \& Brunetta, F. (2018). The individual in society (3rd ed.). Dubuque, Iowa: Kendall/Hunt.

Brunetta, F., \& Reio, T. (2016). How welcome are we? Immigrants as targets of uncivil behavior. In R. Mizzi, T. Rocco, \& S. Shore (Eds.), Disrupting adult and community education: Teaching, learning, and working in the periphery (pp. 227242). Albany, NY: SUNY Press.

Grenier, G., \& Brunetta, F. (2015).The individual in society (2nd ed.). Dubuque, Iowa: Kendall/Hunt. 